EDITORIAL BOARD

ANTONIO CARCATERRA

ERIC A. CARLEN

FRANCESCO DELL'ISOLA

RAFFAELE ESPOSITO

ALBERT FANNJIANG

Gilles A. FranCFORT

Pierangelo MARCATI

JEAN-JACQUES MARIGO

PETER A. MARKOWICH

MARTIN OSTOJA-STARZEWSKI

PIERRE SEPPECHER

DAVID J. STEIGMANN

PAUl STEINMANN

PierRe M. SuQueT

MANAGING EDITORS

MICOL AMAR

CORRADO LATTANZIO

ANGELA MADEO

MARTIN OSTOJA-STARZEWSKI

ADVISORY BOARD

ADNAN AKAY

Holm AltenBaCH

MICOL AMAR

HARM ASKES

TEODOR ATANACKOVIĆ

VICTOR BERDICHEVSKY

GUY BOUCHITTÉ

ANDREA BRAIDES

ROBERTO CAMASSA

MAURO CARFORE

ERIC DARVE

FELIX DARVE

ANNA DE MASI

GianPiEtro DEL Piero

EMMANUELE Di BENEDETTO

BERNOLD FIEDLER

IRENE M. GAMBA

DAVID Y. GAO

SERGEY GAVRILYUK

TIMOTHY J. HEALEY

DOMINIQUE JEULIN

ROGER E. KHAYAT

CORRADO LATTANZIO

ROBERT P. LIPTON

ANGELO LUONGO

ANGELA MADEO

JUAN J. MANFREDI

CARLO MARCHIORO

GÉRARD A. MAUGIN

ROBERTO NATALINI PATRIZIO NEFF

ANDREY PIATNITSKI

ERRICO PRESUTTI

MARIO PULVIRENTI

LUCIO RUSSO

Miguel A. F. SANJUAN

PATRICK SElVADURAI

ALEXANDER P. SEYRANIAN

MIROSLAV ŠILHAVÝ

GUIDO SWEERS

ANTOINETTE TORDESILLAS

LEV TRUSKINOVSKY

JUAN J. L. VELÁZQUEZ VINCENZO VESPRI ANGELO VULPIANI msp.org/memocs

Università di Roma "La Sapienza", Italia

Rutgers University, USA

(CO-CHAIR) Università di Roma "La Sapienza", Italia

(TREASURER) Università dell'Aquila, Italia

University of California at Davis, USA

(CO-CHAIR) Université Paris-Nord, France

Università dell'Aquila, Italy

École Polytechnique, France

DAMTP Cambridge, UK, and University of Vienna, Austria

(CHAIR MANAGING EDITOR) Univ. of Illinois at Urbana-Champaign, USA

Université du Sud Toulon-Var, France

University of California at Berkeley, USA

Universität Erlangen-Nürnberg, Germany

LMA CNRS Marseille, France

Università di Roma "La Sapienza", Italia

Università dell'Aquila, Italy

Université de Lyon-INSA (Institut National des Sciences Appliquées), France

(CHAIR MANAGING EDITOR) Univ. of Illinois at Urbana-Champaign, USA

Carnegie Mellon University, USA, and Bilkent University, Turkey

Otto-von-Guericke-Universität Magdeburg, Germany

Università di Roma "La Sapienza", Italia

University of Sheffield, UK

University of Novi Sad, Serbia

Wayne State University, USA

Université du Sud Toulon-Var, France

Università di Roma Tor Vergata, Italia

University of North Carolina at Chapel Hill, USA

Università di Pavia, Italia

Stanford University, USA

Institut Polytechnique de Grenoble, France

Università dell'Aquila, Italia

Università di Ferrara and International Research Center MEMOCS, Italia

Vanderbilt University, USA

Freie Universität Berlin, Germany

University of Texas at Austin, USA

Federation University and Australian National University, Australia

Université Aix-Marseille, France

Cornell University, USA

École des Mines, France

University of Western Ontario, Canada

Università dell' Aquila, Italy

Louisiana State University, USA

Università dell'Aquila, Italia

Université de Lyon-INSA (Institut National des Sciences Appliquées), France University of Pittsburgh, USA

Università di Roma "La Sapienza”, Italia

Université Paris VI, France

Istituto per le Applicazioni del Calcolo "M. Picone", Italy

Universität Duisburg-Essen, Germany

Narvik University College, Norway, Russia

Università di Roma Tor Vergata, Italy

Università di Roma "La Sapienza”, Italia

Università di Roma “Tor Vergata”, Italia

Universidad Rey Juan Carlos, Madrid, Spain

McGill University, Canada

Moscow State Lomonosov University, Russia

Academy of Sciences of the Czech Republic

Universität zu Köln, Germany

University of Melbourne, Australia

École Polytechnique, France

Bonn University, Germany

Università di Firenze, Italia

Università di Roma La Sapienza, Italia

MEMOCS (ISSN 2325-3444 electronic, 2326-7186 printed) is a journal of the International Research Center for the Mathematics and Mechanics of Complex Systems at the Università dell'Aquila, Italy.

Cover image: "Tangle” by $\odot$ John Horigan; produced using the Context Free program (contextfreeart.org).

PUBLISHED BY

7 mathematical sciences publishers

nonprofit scientific publishing

http://msp.org/

(C) 2018 Mathematical Sciences Publishers 


\title{
AROUND TWO THEOREMS AND A LEMMA BY LUCIO RUSSO
}

\author{
ITAI BENJAMINI AND GIL KALAI
}

\begin{abstract}
We describe two directions of study following early work of Lucio Russo. The first direction follows the famous Russo-Seymour-Welsh (RSW) theorem. We describe an RSW-type conjecture by the first author which, if true, would imply a coarse version of conformal invariance for critical planar percolation. The second direction is the study of "Russo's lemma" and "Russo's 0-1 law" for threshold behavior of Boolean functions. We mention results by Friedgut, Bourgain, and Hatami, and present a conjecture by Jeff Kahn and the second author, which may allow applications for finding critical probabilities.
\end{abstract}

\section{Introduction}

We have not met Lucio Russo in person but his mathematical work has greatly influenced our own and his wide horizons and interests in physics, mathematics, philosophy, and history have greatly inspired us. We describe here two directions of study following early work of Russo. The first section follows the famous Russo-Seymour-Welsh theorem regarding critical planar percolation. The second section follows the basic "Russo's lemma" and the deep "Russo's 0-1 law". In each direction we present one central conjecture.

\section{Planar percolation}

Consider $\frac{1}{2}$-Bernoulli bond percolation on a square lattice. Russo [1978] and Seymour and Welsh [1978] proved the RSW theorem relating the probability of having an open crossing in a $n \times c n$ rectangle to that of crossing a square. In particular, their results imply that

the probability of critical Bernoulli percolation crossing a long rectangle is bounded away from zero and depends only on the aspect ratio.

\section{Communicated by Raffaele Esposito.}

Work of the second author supported in part by ERC advanced grant 320924 .

MSC2010: 60K35, 05C80, 30F10, 68QXX, 82B43.

Keywords: percolation, Russo-Seymour-Welsh theorem, Russo's lemma, Russo's 0-1 law, conformal uniformization, discrete isoperimetry. 
This fundamental fact was crucial in Kesten's proof [1980] that the critical probability for planar percolation is $\frac{1}{2}$, and has been used and extended to a variety of models using clever proofs. But until recently all proofs have depended on rotational symmetry. Vincent Tassion [2016] recently proved the RSW statement under various sets of weaker assumptions, and this has been the key to solving several known problems. On a personal note, we mention that the RSW lemma was essential in controlling the influence of a fixed edge on the crossing event, allowing us to establish, jointly with Oded Schramm, noise sensitivity of critical percolation; see [Benjamini et al. 1999; Garban and Steif 2015].

What about an RSW-type result for more general planar graphs going beyond Euclidean lattices and tessellations?

In what follows we suggest a conjectural extension of the RSW theorem to general planar triangulations. The motivation comes from conformal uniformization; see [Benjamini 2015].

There are strong ties between critical planar percolation and conformal geometry. In [Smirnov 2001] the scaling limit of critical Bernoulli site percolation on the triangular lattice was proved to be conformally invariant. Benjamini [2015] gave a far-reaching conjecture relating percolation and conformal uniformization and derived it from the conjectural extension of the RSW theorem.

A generalized $R S W$ conjecture. Tile the unit square with (possibly infinitely many) squares of varying sizes so that at most three squares meet at corners. That is, the dual graph is a triangulation. Color each square black or white with equal probability independently.

Conjecture 2.1. There is a universal $c>0$ such that the probability of a black left-right crossing is bigger than $c$.

At the moment we do not have a proof of the conjecture even when the squares are colored black with probability $\frac{2}{3}$. Behind the conjecture is a coarse version of conformal invariance. That is, the crossing probability is bounded away from zero and one if the tile shapes are uniformly close to circles (rotation invariance), and the squares can be of different sizes (dilation invariance). If true, the same should hold for a tiling or a packing of a triangulation, with a set of shapes that are of bounded Hausdorff distance to circles.

If the answer to Conjecture 2.1 is affirmative, this will imply (see [Benjamini 2015]) the following: Let $G$ be the 1-skeleton of a bounded degree triangulation of an open disk. Assume $G$ is transient for the simple random walk; then $\frac{1}{2}$-Bernoulli site percolation on $G$ admits infinitely many infinite clusters almost surely. We do not know this even for any $p$-Bernoulli percolation with $1>p>\frac{1}{2}$. In [Benjamini and Schramm 1996b] it is shown that such triangulations result in square tilings as in the conjecture. The proof there is an analogue of the RSW phenomenon 
for a simple random walk on the triangulation. We speculate that $\frac{1}{2}$-Bernoulli site percolation on $G$ admits infinitely many infinite clusters almost surely if and only if $G$ is transient.

How does the influence of a square in the tiling on the crossing probability at $p=\frac{1}{2}$ relate to its area? Establishing a high-dimensional version of the RSW lemma is a well-known and very important open problem. Dan Asimov and Dylan Thurston (private communication) worked out a $2 k$-dimensional model with duality but not yet with RSW. Informally look at the critical $p$ for a full infinite surface and prove RSW for plaquettes in cubes. That is (for $d=3$, say), if the probability of no open path from top to bottom in an $n \times n \times n$ box is at least $\frac{1}{2}$, then there is no open path from top to bottom in a cube $2 n \times 2 n \times n$ with probability bounded away from 0 independently of $n$.

A comment on large graphs and percolation. In the category of planar graphs, in view of (discrete) conformal uniformization, transience (equivalently conformal hyperbolicity) is a natural notion of largeness. In the context of Cayley graphs, nonamenability serves as a notion of large Cayley graphs. Thus the still open conjecture [Benjamini and Schramm 1996a] that there is a nonempty interval of $p$ 's such that $p$-Bernoulli percolation admits infinitely many infinite clusters if and only if the group is nonamenable shares some flavor with Conjecture 2.1: both suggest that a graph is large provided there is a phase with infinitely many infinite clusters.

\section{Isoperimetric inequalities and Russo's 0-1 law}

We endow the discrete cube $\Omega_{n}=\{-1,1\}^{n}$ with the product probability measure $\mu_{p}$, where the probability for each bit to be 1 is $p$. A Boolean function $f$ is a function from $\Omega_{n}$ to $\{-1,1\}$, and $f$ is monotone if changing the value of a variable from -1 to 1 does not change the value of $f$ from 1 to -1 . The influence of the $k$-th variable on $f$, denoted by $I_{k}^{p}(f)$, is the probability that changing the $k$-th variable will change the value of $f$. The total influence is $I^{p}(f)=\sum_{k=1}^{n} I_{k}^{p}(f)$. We denote $\mu_{p}(f)=\mu_{p}\{x: f(x)=1\}$, and write $\operatorname{Var}_{p}(f)=4 \mu_{p}(f)\left(1-\mu_{p}(f)\right)$. (If $p=\frac{1}{2}$ we omit the superscript/subscript $p$.)

A basic result in extremal and probabilistic combinatorics going back to Harper (and others) is the isoperimetric inequality. For the measure $\mu_{p}$ the isoperimetric relation takes the form (see, e.g., [Kahn and Kalai 2007; Kalai 2016]):

Theorem 3.1.

$$
p I^{p}(f) \geq \mu_{p}(f) \log _{p}\left(1 / \mu_{p}(f)\right) .
$$

If $f$ is monotone then $\mu_{p}(f)$ is a monotone function of $p$. Fixing a small $\epsilon>0$, the threshold interval of $f$ is the interval $[p, q]$ where $\mu_{p}(f)=\epsilon$ and $\mu_{q}(f)=1-\epsilon$. A fundamental lemma by Russo [1982] and Margulis [1974] 
asserts that for a monotone Boolean function $f$,

$$
d \mu_{p}(f) / d p=I^{p}(f) .
$$

The deep Russo's 0-1 law [1982] asserts informally that the threshold interval of a Boolean function is of size $o(1)$ if all variables have $o(1)$-influence. In view of the Russo-Margulis lemma, understanding the total influence is crucial for understanding the threshold window of a Boolean function. Sharp form of the Russo 0-1 theorem and various related results were proved in the last two decades, and Fourier methods played an important role in these developments. We mention especially the paper by Kahn, Kalai, and Linial [Kahn et al. 1988] and the subsequent papers [Bourgain et al. 1992; Talagrand 1994; Friedgut and Kalai 1996; Friedgut 1998] and the books [Garban and Steif 2015; O’Donnell 2014]. To a large extent, this study is centered around the following problem.

Problem. Understand the structure of Boolean functions of $n$ variables for which

$$
I^{p}(f) \leq K \frac{1}{p} \mu_{p}(f) \log _{p}\left(1 / \mu_{p}(f)\right) .
$$

We will quickly describe some main avenues of research and central results regarding this problem. For a more detailed recent survey, see [Kalai 2016].

(1) For the case where both $p$ and $\mu_{p}(f)$ are bounded away from zero and one (or even when $\log (1 / p) / \log n \rightarrow 0)$ and $K$ is bounded, Friedgut [1998] proved that such functions are approximately "juntas"; namely, they are determined (with high probability) by their values on a fixed bounded set of variables. This result can be seen as a sharp form of Russo's 0-1 law and it has a wide range of applications.

(2) For the case where $K$ is bounded, $\mu_{p}(f)$ is bounded away from zero and one, but $\log (1 / p) / \log n$ is bounded away from zero, there are important theorems by Friedgut [1999] and Bourgain [1999] (see below) and Hatami [2012]. These results have important applications for proving sharp threshold theorems. Hatami's work is based on the important, if mysterious, notion of pseudojuntas.

(3) The case where $K$ is bounded and $\mu_{p}(f)$ is small is wide open. This case is important on its own and may have some applications for finding the critical probability; see Conjecture 3.3.

(4) Cases where $K=1+\epsilon$ are of different nature and are also of much interest. See [Ellis 2011], for example; work in progress of Ellis and N. Lifshitz is also relevant.

(5) There are few results regarding the case where $K$ is unbounded and especially when $K$ grows quicker than $\log n$. (One such result is by Bourgain and 
Kalai [1997] for functions with various forms of symmetry.) This is of great interest already when both $p$ and $\mu_{p}(f)$ are bounded away from zero and one.

We turn to a theorem of Bourgain and a related and far-reaching conjecture.

Theorem 3.2 [Bourgain 1999]. There exists $\epsilon>0$ with the following property: For every $C$ there is $K(C)$ such that if $I^{p}(f)<p C$, then there exists a subset $R$ of variables $|R| \leq K(C)$ such that

$$
\mu_{p}\left(x: f(x)=1 \mid x_{i}=1, i \in S\right)>(1+\epsilon) \mu_{p}(f) .
$$

Conjecture 3.3 [Kahn and Kalai 2007, Conjecture 6.1(a)]. There exists $\epsilon>0$ with the following property: For every $C$ there is $K(C)$ such that if $I^{p}(f)<$ $p C \mu_{p}(f) \log \left(1 / \mu_{p}(f)\right)$ then there exists a subset $R$ of variables $|R| \leq K(C)$ $\log \left(1 / \mu_{p}(f)\right)$ such that

$$
\mu_{p}\left(x: f(x)=1 \mid x_{i}=1, i \in S\right)>(1+\epsilon) \mu_{p}(f) .
$$

Several attempted stronger conjectures (such as Conjectures 6.1(b), 6.1(c) in [Kahn and Kalai 2007]) turned out to be incorrect. Conjecture 3.3 was motivated by a far reaching conjecture from [Kahn and Kalai 2007] relating two notions of a threshold for random graphs. Related questions were raised in [Talagrand 2010]. We conclude with another approach for understanding Boolean functions with small influence. The first step is the important Fourier-Walsh expansion. Every Boolean function $f$ can be written as a square free polynomial $f=\sum \hat{f}(S) x_{S}$, where $x_{S}=\prod\left\{x_{i}: i \in S\right\}$. (The coefficients $\hat{f}(S)$ are called the Fourier coefficients of $f$.) It is easy to verify that $\sum \hat{f}^{2}(S)=1$ and that $\sum \hat{f}^{2}(S)|S|=I(f)$. Therefore:

Proposition 3.4. For every $\epsilon>0$, a Boolean function $f$ can be $\epsilon \cdot \operatorname{Var}(f)$-approximated by the sign of a degree-d polynomial where $d=(1 / \epsilon) I(f)$.

However, we note that Boolean functions described as signs of low-degree polynomials may have large total influence. Our next step is to consider the representation of Boolean functions via Boolean circuits. Circuits allow us to build complicated Boolean functions from simple ones, and they have crucial importance in computational complexity. Starting with $n$ variables $x_{1}, x_{2}, \ldots, x_{n}$, a literal is a variable $x_{i}$ or its negation $-x_{i}$. Every Boolean function can be written as a formula in conjunctive normal form, namely as ANDs of ORs of literals. A circuit of depth $d$ is defined inductively as follows: A circuit of depth zero is a literal. A circuit of depth one consists of an OR or AND gate applied to a set of literals. A circuit of depth $k$ consists of an OR or AND gate applied to the outputs of circuits of depth $k-1$. (We can assume that gates in the odd levels are all OR gates and that the gates of the even levels are all AND gates.) The size of a circuit is the number of gates. The famous $\mathrm{NP} \neq \mathrm{P}$ conjecture (in a slightly stronger form) asserts that 
the Boolean function described by the graph property of containing a Hamiltonian cycle cannot be described by a polynomial-size circuit.

A theorem by Boppana [1984] (the monotone case) and Håstad [1989] (the general case) asserts that if $f$ is described by a Boolean circuit of depth $d$ and size $M$ then $I(f) \leq C(\log M)^{d-1}$. We conjecture that functions with low influence can be approximated by low-depth small-size circuits. A function $g \delta$-approximates a function $f$ if $\left|\mathbb{E}(f-g)^{2}\right| \leq \epsilon$.

The next conjecture is slightly extended from one in [Benjamini et al. 1999].

Conjecture 3.5 (Benjamini, Kalai, and Schramm). For some absolute constant $C$ the following holds: For every $\epsilon>0$ a Boolean function $f$ can be $\epsilon \cdot \operatorname{Var}(f)$ approximated by a circuit of depth $d$ and size $M$, where

$$
(\log M)^{C d} \operatorname{Var}(f) \leq I(f) .
$$

\section{Conclusion}

Congratulations Lucio on your remarkable career and contributions and best wishes for the future. It is time for us to meet!

\section{References}

[Benjamini 2015] I. Benjamini, "Percolation and coarse conformal uniformization", 2015. arXiv [Benjamini and Schramm 1996a] I. Benjamini and O. Schramm, "Percolation beyond $\mathbb{Z}^{d}$, many questions and a few answers", Electron. Comm. Probab. 1 (1996), no. 8, 71-82.

[Benjamini and Schramm 1996b] I. Benjamini and O. Schramm, "Random walks and harmonic functions on infinite planar graphs using square tilings", Ann. Probab. 24:3 (1996), 1219-1238.

[Benjamini et al. 1999] I. Benjamini, G. Kalai, and O. Schramm, "Noise sensitivity of Boolean functions and applications to percolation", Inst. Hautes Études Sci. Publ. Math. 90 (1999), 5-43.

[Boppana 1984] R. B. Boppana, “Threshold functions and bounded depth monotone circuits", pp. 475-479 in Proc. 16th Annual ACM Symp. Theory of Computing (Washington, DC, 1984), ACM, New York, 1984.

[Bourgain 1999] J. Bourgain, "On sharp thresholds of monotone properties", J. Amer. Math. Soc. 12 (1999), 1051-1054. Appendix to [Friedgut 1999].

[Bourgain and Kalai 1997] J. Bourgain and G. Kalai, "Influences of variables and threshold intervals under group symmetries”, Geom. Funct. Anal. 7:3 (1997), 438-461.

[Bourgain et al. 1992] J. Bourgain, J. Kahn, G. Kalai, Y. Katznelson, and N. Linial, "The influence of variables in product spaces", Israel J. Math. 77:1-2 (1992), 55-64.

[Ellis 2011] D. Ellis, "Almost isoperimetric subsets of the discrete cube", Combin. Probab. Comput. 20:3 (2011), 363-380.

[Friedgut 1998] E. Friedgut, "Boolean functions with low average sensitivity depend on few coordinates", Combinatorica 18:1 (1998), 27-35.

[Friedgut 1999] E. Friedgut, "Sharp thresholds of graph properties, and the $k$-sat problem", J. Amer. Math. Soc. 12:4 (1999), 1017-1054. 
[Friedgut and Kalai 1996] E. Friedgut and G. Kalai, "Every monotone graph property has a sharp threshold", Proc. Amer. Math. Soc. 124:10 (1996), 2993-3002.

[Garban and Steif 2015] C. Garban and J. E. Steif, Noise sensitivity of Boolean functions and percolation, Cambridge University Press, 2015.

[Håstad 1989] J. Håstad, "Almost optimal lower bounds for small depth circuits", pp. 6-20 in Randomness and Computation, edited by S. Micali, JAI Press, 1989.

[Hatami 2012] H. Hatami, "A structure theorem for Boolean functions with small total influences", Ann. of Math. (2) 176:1 (2012), 509-533.

[Kahn and Kalai 2007] J. Kahn and G. Kalai, "Thresholds and expectation thresholds", Combin. Probab. Comput. 16:3 (2007), 495-502.

[Kahn et al. 1988] J. Kahn, G. Kalai, and N. Linial, "The influence of variables on Boolean functions", pp. 68-80 in Proc. 29th Annual Symp. Foundations of Computer Science (White Plains, NY, 1988), 1988.

[Kalai 2016] G. Kalai, "Boolean functions: influence, threshold and noise”, preprint, 2016, available at https://gilkalai.files.wordpress.com/2017/01/7ecmkalr.pdf. To appear in the Proceedings of the Seventh European Congress of Mathematics.

[Kesten 1980] H. Kesten, "The critical probability of bond percolation on the square lattice equals $\frac{1}{2}$ ”, Comm. Math. Phys. 74:1 (1980), 41-59.

[Margulis 1974] G. A. Margulis, "Probabilistic characteristics of graphs with large connectivity", Probl. Pered. Inform. 10:2 (1974), 101-108. In Russian.

[O’Donnell 2014] R. O’Donnell, Analysis of Boolean functions, Cambridge University Press, 2014.

[Russo 1978] L. Russo, “A note on percolation”, Z. Wahrsch. Verw. Gebiete 43:1 (1978), 39-48.

[Russo 1982] L. Russo, “An approximate zero-one law”, Z. Wahrsch. Verw. Gebiete 61:1 (1982), 129-139.

[Seymour and Welsh 1978] P. D. Seymour and D. J. A. Welsh, "Percolation probabilities on the square lattice", Ann. Discrete Math. 3 (1978), 227-245.

[Smirnov 2001] S. Smirnov, "Critical percolation in the plane: conformal invariance, Cardy's formula, scaling limits", C. R. Acad. Sci. Paris Sér. I Math. 333:3 (2001), 239-244.

[Talagrand 1994] M. Talagrand, “On Russo's approximate zero-one law”, Ann. Probab. 22:3 (1994), $1576-1587$.

[Talagrand 2010] M. Talagrand, "Are many small sets explicitly small?", pp. 13-36 in Proc. 42nd ACM Symp. Theory of Computing (Cambridge, MA, 2010), ACM, New York, 2010.

[Tassion 2016] V. Tassion, "Crossing probabilities for Voronoi percolation", Ann. Probab. 44:5 (2016), 3385-3398.

Received 16 Jan 2017. Revised 3 Jan 2018. Accepted 4 Feb 2018.

ITAI BENJAMINI: itai.benjamini@weizmann.ac.il

Department of Mathematics, Weizmann Institute, Rehovot, Israel

GIL KALAI: kalai@math.huji.ac.il

Einstein Institute of Mathematics, Hebrew University of Jerusalem, Givat Ram Campus, Jerusalem, Israel 



\title{
A STRAIN GRADIENT VARIATIONAL APPROACH TO DAMAGE: A COMPARISON WITH DAMAGE GRADIENT MODELS AND NUMERICAL RESULTS
}

\author{
LuCa Placidi, Emilio Barchiesi ANd ANIL MisRa
}

The global response of experimental uniaxial tests cannot be homogeneous, because of the unavoidable presence of localized deformations, which is always preferential from an energetic viewpoint. Accordingly, one must introduce some characteristic lengths in order to penalize deformations that are too localized. This is what leads to the concept of nonlocal damage models. The nonlocal approach employs nonlocal terms in the internal deformation energy in order to control the size of the localization region. In phase-field models and, in general, in gradient models, dependence of the internal energy upon the first gradient of damage is assumed, while in our approach the nonlocality is given by the dependence of the internal energy upon the second gradient of the displacement field. A discussion of the advantages and challenges of using the gradient of damage and of using the second gradient of the displacement field will be addressed in the present paper. A variational inequality is formulated and partial differential equations (PDEs), boundary conditions (BCs), and Karush-KuhnTucker (KKT) conditions will be derived within the framework of 2D strain gradient damage mechanics. A novel dependence of the stiffness coefficients with respect to the damage field will also be discussed. Further, an explicit derivation of the damage field evolution in loading conditions will be provided. Finally, a numerical technique based on commercial software has been introduced and discussed for a couple of standard problems.

\section{Introduction}

1.1. A short overview. The literature on regularized damage laws, or so-called phase-field models, when the regularization is performed on the damage variable, has become quite intense in these last ten years. It has been proved, e.g., in [Lorentz and Andrieux 2003], that a regularization through the introduction of the gradient of damage allows one to overcome issues related to localization and mesh-dependency. Moreover, rigorous proofs of the convergence of such models

\section{Communicated by Jean-Jacques Marigo.}

MSC2010: 74C05, 74R99.

Keywords: strain gradient, damage mechanics, variational inequality, dissipation, regularization. 
towards the Griffith model of brittle fracture have strengthened such choice of regularization through the gradient of the damage variable; see for example [Ambrosio and Tortorelli 1990; Sicsic and Marigo 2013]. Thus, the nonlocality is customarily given by the dependence of the internal energy $U$ upon the first gradient of damage $\nabla \omega$ [Marigo 1989; Comi 1999; Pham et al. 2011a; Miehe et al. 2016]. A fully nonlocal model, in the Piola-peridynamic framework [dell'Isola et al. 2015a], has also been developed in the literature, like in, e.g., [Bažant and Jirásek 2002; Bažant and Pijaudier-Cabot 1988]. Another interesting and effective approach is due to [Forest 2009]. In the approach presented in this paper the nonlocality is given by the dependence of the internal energy upon the second gradient of the displacement field. In other words, the internal elastic energy per unit volume $U$ is assumed to be a function not only of the strain $G$, but also of its gradient $\nabla G$. This approach is not new in damage continuum mechanics; see also [Peerlings et al. 2001; Mühlich et al. 2013; Zybell et al. 2009; Oliver-Leblond et al. 2016]. Beyond the convergence of some damage gradient models towards the Griffith model for brittle fracture, the main advantage of using the gradient of damage is simply due to the fact that handling the gradient of a scalar (i.e., the damage field) is easier than dealing with the gradient of a tensor (i.e., the strain). A first consequence of this fact is that the number of constitutive parameters for a damage gradient model is lower than that of a strain gradient model, and this will be discussed in more detail in the next subsection. In the remainder of this section we investigate and present two main advantages of using the strain gradient approach. First of all, an interpretation of those boundary conditions that are necessary to ensure the uniqueness of the solution is guaranteed only for strain gradient models and not for damage gradient models. This issue is very important when performing experimental and numerical parameter identification. Secondly, regularization in the elastic phase is achieved only with strain gradient models and not with damage gradient models.

1.2. Number of constitutive parameters. Let us consider for simplicity the isotropic case. For the strain gradient model the number of constitutive coefficients to be identified (in addition to the standard Lamé coefficients) is 5 for the 3D case and 4 for the 2D case. For the damage gradient model, in addition to the standard Lamé coefficients, we have 1 further parameter. In order to identify the constitutive parameters, an experimental procedure is necessary. In this regard, many attempts [Placidi et al. 2015; 2017; Rahali et al. 2016] have been exploited in the framework of strain gradient elasticity.

1.3. Interpretation of boundary conditions. In continuum damage mechanics, the kinematics (see also Figure 1) is defined by both the displacement $u(X, t)$ (or the placement $\chi(X, t))$ and the damage $\omega(X, t)$ fields. In the damage gradient approach one assumes always natural boundary conditions, as is shown in Figure 2, 


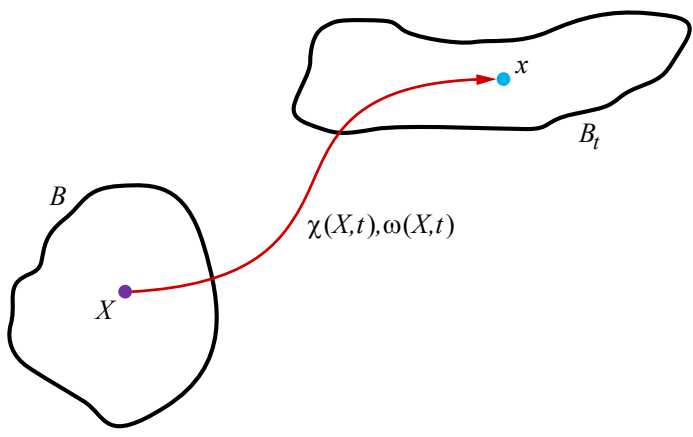

Figure 1. Kinematics in continuum damage mechanics.

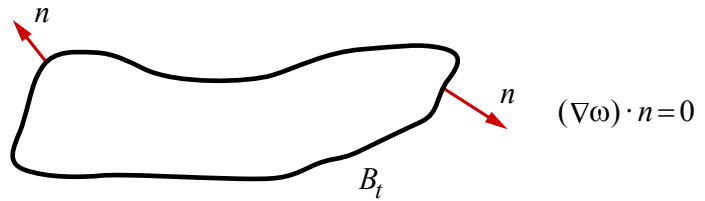

Figure 2. Natural boundary conditions for the damage gradient approach.

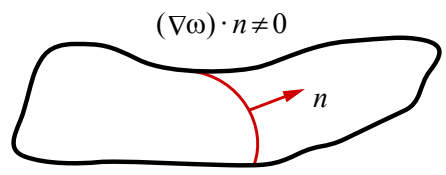

Figure 3. An internal boundary (a line in the present $2 \mathrm{D}$ case) is depicted, where $n$ is one of its unit normals. The internal boundary is chosen in such a way that the projection of the damage gradient on the unit normal $n$ is nonzero.

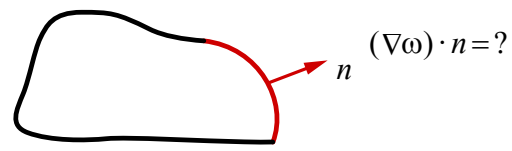

Figure 4. External boundary conditions for the damage gradient approach that guarantee the same solution of the boundary value problem represented in Figure 2.

where $n$ is the external unit normal. With these natural boundary conditions, which are represented in Figure 2, one obtains a solution in terms of the damage field $\omega(X, t)$ such that there exists an internal boundary where

$$
(\nabla \omega) \cdot n \neq 0
$$




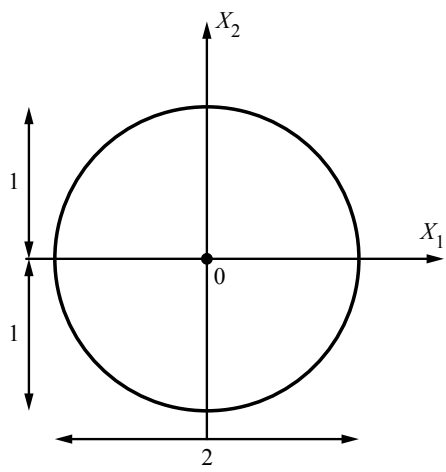

Figure 5. Domain of the elastic problem defined in (3).

Such an internal boundary is depicted, e.g., in Figure 3. Let us now perform a so-called Cauchy-cut over the internal boundary represented in Figure 3 and take the left part in such a way that the unit normal $n$ defines its external unit normal. We now take into account the boundary value problem, as in Figure 4, such that the solutions of the problems represented in Figures 3 and 4 are the same if restricted over the domain of Figure 4. Which kind of boundary conditions should we assume for the damage gradient? If we assume zero natural boundary conditions, then the solutions of the problems in Figures 3 and 4, restricted over the domain of Figure 4, are different. Because of the uniqueness of the solution, in order for the problems in Figures 3 and 4 to have the same solution, we should have nonzero natural boundary conditions. Thus, in the damage gradient approach one faces the problem of interpreting the natural boundary conditions. It is worth noting that the interpretation of the extra boundary conditions in the strain gradient approach, in terms of the normal gradient of displacement and/or double force and in terms of vertex-contact actions, is standard for elastic strain gradient models.

1.4. Regularization of the elastic phase. In order to support the claim that a regularized scheme is necessary also for the elastic phase, we consider the following example (personal communication of Pierre Seppecher). The number of examples of this kind is very large. However, what we show here is simple to conceive and deserves a short illustration. Let us find, among all the displacement fields that satisfy the boundary conditions

$$
u / u(O)=\hat{e}_{3}, \quad u(\partial \Omega)=0,
$$

the solution to the second-gradient elastic problem

$$
\inf \int_{\Omega}\|\nabla \nabla \boldsymbol{u}\|^{2},
$$


where $\Omega \subseteq \mathbb{R}^{2}$ is the circle of radius equal to 1 in Figure 5 and $\hat{e}_{3}$ is the out-of-plane unit vector. It is possible to prove that the solution to (3) is

$$
u=\frac{2}{3} r^{2} \ln r-r^{2}+1
$$

and that the infimum, which is in fact a minimum, is

$$
\int_{\Omega}\|\nabla \nabla \boldsymbol{u}\|^{2}=\pi \frac{16}{3} \text {. }
$$

This means that the energy attained in correspondence of the solution is finite, as one would expect. If the energy to be minimized, among all the displacement fields satisfying the (2), is of first-gradient type, e.g., it is given by

$$
\inf \int_{\Omega}\|\nabla \boldsymbol{u}\|^{2}
$$

an explicit analytical solution can still be found and reads as

$$
u=\lim _{\varepsilon \rightarrow 0} \frac{\ln r}{\ln \varepsilon} .
$$

In this case, the infimum is

$$
\int_{\Omega}\|\nabla \boldsymbol{u}\|^{2}=-\lim _{\varepsilon \rightarrow 0} \frac{2 \pi}{\ln \varepsilon}=0,
$$

which means that the energy attained in correspondence of the solution is zero, a fact that is clearly not reasonable on a physical ground.

\section{The variational inequality and the derivation of governing equations}

In order to formulate governing equations for nonstandard models, it is useful to use a variational procedure. The reason for such a choice is that the definition of those boundary conditions that guarantee uniqueness of the solution is straightforward in this way. A variational principle of maximum plastic work has been derived already by Hill [1948]. Further contributions are due to, among others, [Maier 1970; Bažant 1980; Bourdin et al. 2008; Pham et al. 2011b; Marigo 1989; Amor et al. 2009; Pham and Marigo 2010a; 2010b; Reddy 2011a; 2011b].

2.1. Kinematics of the model. As shown in Figure 1, the kinematics of the model is given by the displacement field $u$, which is an observable state variable

$$
u:\left(\mathbb{R}^{2} \supseteq \mathscr{B},[0, T]\right) \rightarrow \mathbb{R}^{2},
$$

and by the damage field $\omega$, which is an internal state variable

$$
\omega:\left(\mathbb{R}^{2} \supseteq \mathscr{B},[0, T]\right) \rightarrow[0,1],
$$


with $\omega=0$ corresponding to the undamaged material and $\omega=1$ to the failure state. Further, we don't take into account any healing mechanism, and this introduces the unilateral (entropic) constraint

$$
\dot{\omega} \geq 0
$$

2.2. The variational inequality. We assume a total deformation energy functional $\mathscr{E}(u, \omega)$ of the kind which has been discussed so far (i.e., including strain gradient). Thus, we evaluate its variation $\delta \mathscr{E}(u, \omega, \delta u, \delta \omega)$. Finally, the variational inequality

$$
\delta \mathscr{E}(u, \omega, \dot{u}, \dot{\omega}) \leq \delta \mathscr{E}(u, \omega, v, \beta) \quad \text { for all } v \text { and for all } \beta \geq 0
$$

is assumed for any admissible virtual velocity fields $\beta$ and $v$. As remarked in [Marigo 1989], inequality (12) states that the actual energy release rate is not smaller than any possible one. Thus, it constitutes a kind of principle of maximum energy release rate.

2.3. The total energy functional in the strain gradient damage $2 D$ case. The total energy functional which is here investigated is defined as

$$
\begin{aligned}
\mathscr{E}(u, \omega)=\int_{\mathscr{B}}\left[U(G, \nabla G, \omega)-b^{\mathrm{ext}} \cdot u-m^{\mathrm{ext}} \cdot \nabla u\right] d A \\
\quad-\int_{\partial \mathscr{B}}\left[t^{\mathrm{ext}} \cdot u+\tau^{\mathrm{ext}} \cdot[(\nabla u) n]\right] d s-\int_{[\partial \partial \mathscr{B}]} f^{\mathrm{ext}} \cdot u,
\end{aligned}
$$

where a standard second-gradient elastic energy, with $G$ the symmetric part of the displacement gradient, has been complemented with an isotropic local damage dissipation term. The $2 \mathrm{D}$ isotropic quadratic internal deformation energy density functional accounting for damage is

$$
U(G, \nabla G, \omega)=U_{e}(G, \nabla G, \omega)+\frac{k}{2} \omega^{2},
$$

where $k$ is the resistance to damage. The elastic part $U_{e}(G, \nabla G, \omega)$ of the internal energy that is here considered is

$$
\begin{aligned}
U_{e}(G, \nabla G, \omega)=2 \mu G_{12}^{2}+\frac{1}{2} \lambda\left(G_{11}+G_{22}\right)^{2}+\mu\left(G_{11}^{2}+G_{22}^{2}\right) \\
+\frac{B}{2}\left(G_{11,1}{ }^{2}+G_{22,2}{ }^{2}\right)+2 A\left(G_{12,1}{ }^{2}+G_{12,2}{ }^{2}\right) \\
+\left(\frac{3 A}{2}-B+C+2 D\right)\left(G_{11,2}^{2}+G_{22,1}{ }^{2}\right) \\
+(A+B-2 C)\left(G_{11,1} G_{12,2}+G_{12,1} G_{22,2}\right) \\
+(-4 A+2 B-4 D)\left(G_{12,2} G_{22,1}+G_{11,2} G_{12,1}\right) \\
+\left(-\frac{A}{2}-\frac{B}{2}+C+2 D\right)\left(G_{11,2} G_{22,2}+G_{11,1} G_{22,1}\right),
\end{aligned}
$$


where the stiffness coefficients $\lambda, \mu, A, B, C$, and $D$ all depend upon damage $\omega$. The dependence of the elastic coefficients upon damage is illustrated in the next subsection.

2.4. Dependence of the elastic coefficients upon damage. In our model Lamé constants are, as it is customarily assumed in damage mechanics, a decreasing function of damage,

$$
\lambda=\lambda_{0}(1-\omega), \quad \mu=\mu_{0}(1-\omega),
$$

while second-gradient elastic stiffnesses are increasing with increasing damage,

$$
A=A_{0}(1+n \omega), \quad B=B_{0}(1+n \omega), \quad C=C_{0}(1+n \omega), \quad D=D_{0}(1+n \omega), \quad n \in \mathbb{R} .
$$

The reason for such a choice is that the state of damage is by itself a kind of measure of the microstructures of the continuum. When modeling some classes of phenomena (e.g., the behavior of laminate composites, where damage is spreading without localizing too much), it is reasonable to conceive a model in which the postulated dependencies (17) of $A, B, C$, and $D$ upon damage are appropriate. For further details the reader is referred to the complete formulation in [Placidi 2015; 2016]. Moreover, in Section 4 some numerical results show the sensitivity of a certain solution upon the parameter $n$. Finally, it is worth noting that, in order to study the fracture propagation, equations (17) should change. However, this will be the topic of another work.

2.5. Derivation of governing partial differential equations. It is possible to prove that the variational inequality (12) reduces to the usual balance of momenta when arbitrary variations $\delta u$ and no variations $\delta \omega$, i.e., $\delta \omega=0$, are considered:

$$
\delta \mathscr{E}(u, \omega, \delta u, \delta \omega=0)=0 .
$$

By applying the localization theorem we get the system of PDEs

$$
\left(S_{i j}-T_{i j h, h}\right)_{, j}+b_{i}^{\text {ext }}-m_{i j, j}^{\text {ext }}=0 \quad \text { for all } X \in \mathscr{B},
$$

where stress and hyperstress are defined as

$$
S_{i j}=\frac{\partial U}{\partial G_{i j}}, \quad T_{i j h}=\frac{\partial U}{\partial G_{i j, h}} .
$$

2.6. Derivation of boundary conditions. For those points of $\partial \mathscr{B} \backslash[\partial \partial \mathscr{B}]$ where kinematical constraints on $u$ are not given, i.e., where $\delta u \neq 0$, we have the natural boundary conditions $t-t^{\mathrm{ext}}-m^{\mathrm{ext}} n=0$. For those points of the $\partial \mathscr{B} \backslash[\partial \partial \mathscr{B}]$ where kinematical constraints on $\nabla u n$ are not given, i.e., where $\delta \nabla u n \neq 0$, we have the

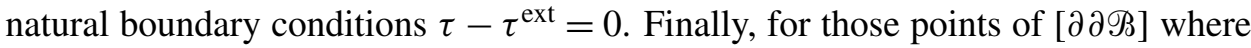


kinematical constraints on $u$ are not given, i.e., where $\delta u \neq 0$, we have the natural boundary conditions $f-f^{\text {ext }}=0$. For further details, i.e., for proper definitions of the contact actions $t, \tau$, and $f$, the reader is referred to the complete formulation in [Placidi et al. 2015; Placidi and El Dhaba 2017].

2.7. Derivation of the Karush-Kuhn-Tucker conditions. By choosing $v=\dot{u}$ and $\beta=0$ (followed by the choice $\beta=2 \dot{\omega}$ and then by the choice $\beta=\dot{\omega}$ ) in the variational inequality (12), it is possible to prove, by localization arguments, that $\frac{\partial U}{\partial \omega}$ and/or $\dot{\omega}$ (which is always nonnegative, i.e., $\dot{\omega} \geq 0$ ) must vanish for each point $X$ of $\mathscr{B}$ and time $t$

$$
\frac{\partial U}{\partial \omega} \dot{\omega}=0 \quad \text { for all } X \in \mathscr{B} \text {. }
$$

Thus, we are able to derive the so-called Karush-Kuhn-Tucker (KKT) conditions for damage mechanics simply from the variational principle (12).

2.8. Comments. For a fixed $\omega$, the behavior is (linear and) elastic. However, since $\omega$ evolves (quasistatically), the global behavior is inelastic and the effective Young's modulus is proportional to $(1-\omega)$. This corresponds to a global softening behavior. Further, given the choice of the dissipation energy (i.e., quadratic dependence upon damage), damage will increase from the very beginning and no purely elastic behavior is observed as if, e.g., a linear dependence upon damage was assumed. It is crucial, even if redundant, to remark that our model accounts for the fact that localization of strain and damage consists of a two-way interaction: localization of strain implies localization of damage and vice versa. Anyway, contrarily to what is done usually in damage mechanics, accounting for nonlocal behavior is not encoded in the (local) damage term (indeed this is not a phase field model, i.e., nonlocal/gradient damage). Accounting for nonlocal behavior is encoded in the dependence of the strain energy upon the strain gradient (advantages and challenges of this approach were explained in the previous section). It is possible to show that, without nonlocal terms, concentration of stress (strain) leads to a burst of damage (up to 1) in these very localized regions. Consequently, the first-gradient model works only for moderate levels of mean damage, being unable to capture, for instance, the softening process. Further, since strain gradient terms make us "pay" for the localization of strain (stress), they play the role of "limiters" against brutal failure. Thus, the model works up to higher levels of mean damage, being able to capture the softening process.

\section{Solution algorithm in incremental form}

Since $\dot{\omega} \geq 0$, KKT conditions (21) imply that

$$
\frac{\partial U}{\partial \omega}=0 \quad \vee \quad \dot{\omega}=0 \quad \text { for all } X \in \mathscr{B} .
$$


Then, we define a damage threshold $\bar{\omega}(G, \nabla G)$ such that

$$
\frac{\partial U}{\partial \omega}(G, \nabla G, \bar{\omega}(G, \nabla G))=0 .
$$

With the prescriptions (15) on the functional dependence of the elastic internal energy upon the strain and the strain gradient tensors, (14) on the functional dependence of the dissipation energy upon the entropic damage variable, and (16)-(17) on the functional dependence of the first- and second-gradient elastic stiffnesses upon the entropic damage variable, we obtain the form for the damage threshold

$$
\begin{aligned}
\bar{\omega}(X, t) & =\frac{\lambda_{0}+2 \mu_{0}}{k}\left(u_{1,1}^{2}+u_{2,2}^{2}\right)+2 \frac{\mu_{0}}{k} u_{1,2} u_{2,1}+\frac{\mu_{0}}{k}\left(u_{1,2}^{2}+u_{1,1}^{2}\right)+2 \frac{\lambda_{0}}{k} u_{1,1} u_{2,2} \\
- & n \frac{A_{0}}{k}\left(u_{1,22}^{2}+u_{2,11}^{2}\right)-n \frac{B_{0}}{k}\left(u_{1,11}^{2}+u_{2,22}^{2}\right)-n \frac{C_{0}}{k}\left(u_{1,12}^{2}+u_{2,12}^{2}\right) \\
- & 2 n \frac{D_{0}}{k}\left(u_{1,11} u_{2,12}+u_{2,22} u_{1,12}\right)-n \frac{B_{0}-C_{0}+A_{0}}{k}\left(u_{1,11} u_{1,22}+u_{2,11} u_{2,22}\right) \\
- & 2 n \frac{B_{0}-A_{0}-D_{0}}{k}\left(u_{1,12} u_{2,11}+u_{1,22} u_{2,12}\right) .
\end{aligned}
$$

First of all, an initial condition for both displacement and damage is assigned as $u(X, 0)=u_{0}(X)=0 \quad$ for all $X \in \mathscr{B}, \quad \omega(X, 0)=\omega_{0}=0 \quad$ for all $X \in \mathscr{B}$.

Once the initial condition is assigned, the displacement field $u_{i}(X)$ for the $i$-th step (with $i \in \mathbb{N}$ ) is derived from (18) as

$$
u_{i}=\arg \min _{u: \mathscr{乃} \rightarrow \mathbb{R}^{2}} \mathscr{E}\left(u, \omega_{i-1}\right),
$$

and the damage field $\omega_{i}(X)$ for the $i$-th step is derived from (21) as

$$
\omega_{i}=\max \left(\bar{\omega}\left(G_{i}, \nabla G_{i}\right), \omega_{i-1}\right),
$$

where here we intend $u_{i}$ and $\omega_{i}$ to be the values, at a certain point, of the displacement $u$ and damage $\omega$ at the time step $t_{i}$. It is worth noting that an a priori discretization of the time variable, that in the present quasistatic case is interpreted as an order parameter, must be performed.

The incremental formulation has been implemented in MATLAB. For simplicity, we performed only displacement-controlled numerical experiments and, at each step, the minimization problem in (26) is approximated by means of the weak form package of the FEM software COMSOL Multiphysics. The mesh is triangular, and it is Delaunay-tessellated (maximum element size $3.0 \times 10^{-4} \mathrm{~m}$ and minimum element size $\left.6.0 \times 10^{-7} \mathrm{~m}\right)$. When strain gradient constitutive coefficients $A=$ $B=C=D=0$ are null, quadratic Lagrangian shape functions are employed while, when they are greater than zero, cubic Hermite shape functions are used. The Newton-Raphson method is used to numerically solve the algebraic system 


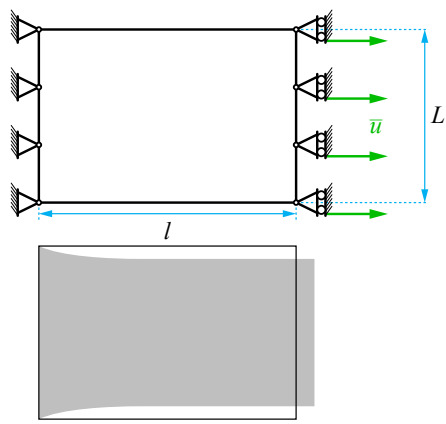

Figure 6. Tensile test (plain plate): reference domain with a schematic of BCs (top); vertical displacement of the deformed configuration for a linear elastic isotropic homogeneous body (bottom) is emphasized more than the horizontal displacement.

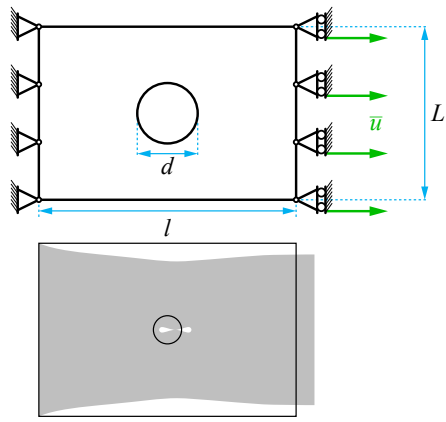

Figure 7. Tensile test (perforated plate): reference domain with a schematic of BCs (top); vertical displacement of the deformed configuration for a linear elastic isotropic homogeneous body (bottom) is emphasized more than the horizontal displacement.

coming from the Galerkin approximation. The computational time for each step is approximately $40 \mathrm{~s}$ with an Intel Core i7-6700HQ CPU at $2.60 \mathrm{GHz}$ and $16 \mathrm{~GB}$ RAM machine.

\section{Tensile tests of plain and perforated plate}

We investigate two simple geometries: a plain rectangle, like the one in Figure 6, and a perforated rectangle, like the one in Figure 7.

Numerical simulations have been performed with the constitutive coefficients illustrated in Table 1, where the Lamé coefficients $\lambda$ and $\mu$ depend in the standard way upon the Young's modulus and Poisson's ratio given in Table 1. Further, $L$ 


\begin{tabular}{|ccccc|}
\hline $\begin{array}{c}\text { Young's modulus }(Y) \\
75 \mathrm{GPa}\end{array}$ & $\begin{array}{c}\text { Poisson's ratio } \\
0.11\end{array}$ & $\begin{array}{c}k \\
75 \mathrm{kPa}\end{array}$ & $\begin{array}{c}L \\
20 \mathrm{~mm}\end{array}$ & $\begin{array}{c}l \\
30 \mathrm{~mm}\end{array}$ \\
\hline$\alpha_{1}^{0}, \alpha_{2}^{0}, \alpha_{3}^{0}, \alpha_{4}^{0}$ & $\alpha_{5}^{0}$ & $n$ & $d$ & \\
$m \cdot Y \cdot 1 \mathrm{~mm}^{2}$ & $\alpha_{1}^{0} / 2$ & 0 or 1 & $0.33 \mathrm{~mm}$ & \\
\hline
\end{tabular}

Table 1. Numerical values which are used in simulations.

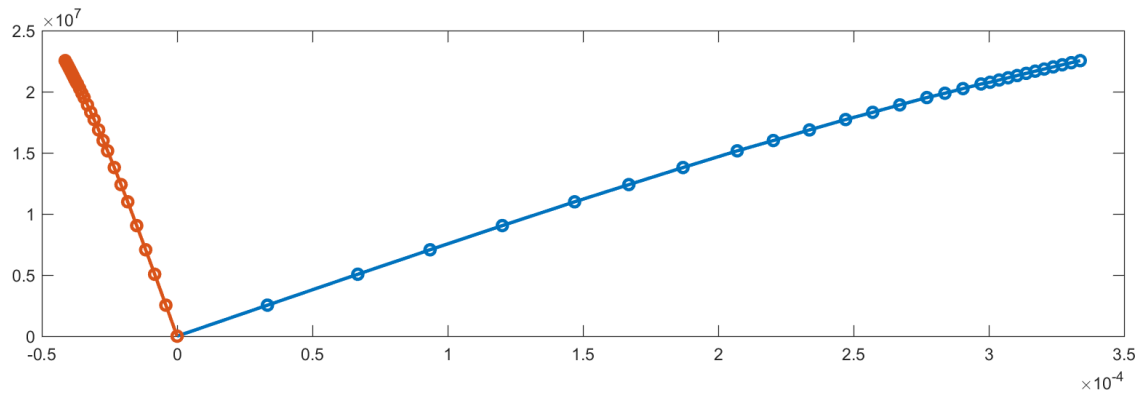

Figure 8. Tensile test (plain plate): $m=0 . \quad S_{11}(\mathrm{~Pa})$ (right side midpoint) versus $G_{11}$ (right side midpoint) (blue); $S_{11}(\mathrm{~Pa})$ (right side midpoint) versus $G_{22}$ (right side midpoint) (orange); $l=30 \mathrm{~mm} ; m=0 ; n=1$.

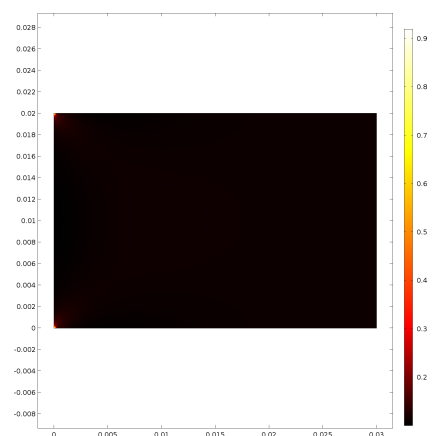

Figure 9. Tensile test (plain plate): $m=0$. Color map of $\omega$ at failure point; $l=30 \mathrm{~mm} ; m=0 ; n=1$. No boundary layer. Burst of damage in very localized zones.

and $l$ are the lengths of the sides of the rectangle and the undamaged secondgradient stiffnesses $A_{0}, B_{0}, C_{0}$, and $D_{0}$ are related to the 5 Mindlin's 3D coefficients illustrated in Table 1 as

$$
\left(\begin{array}{l}
A_{0} \\
B_{0} \\
C_{0} \\
D_{0}
\end{array}\right)=\left(\begin{array}{lllll}
0 & 0 & 2 & 2 & 2 \\
8 & 2 & 8 & 4 & 8 \\
2 & 1 & 1 & 3 & 5 \\
3 & 1 & 2 & 0 & 0
\end{array}\right)\left(\begin{array}{l}
\alpha_{1}^{0} \\
\alpha_{2}^{0} \\
\alpha_{3}^{0} \\
\alpha_{4}^{0} \\
\alpha_{5}^{0}
\end{array}\right) .
$$




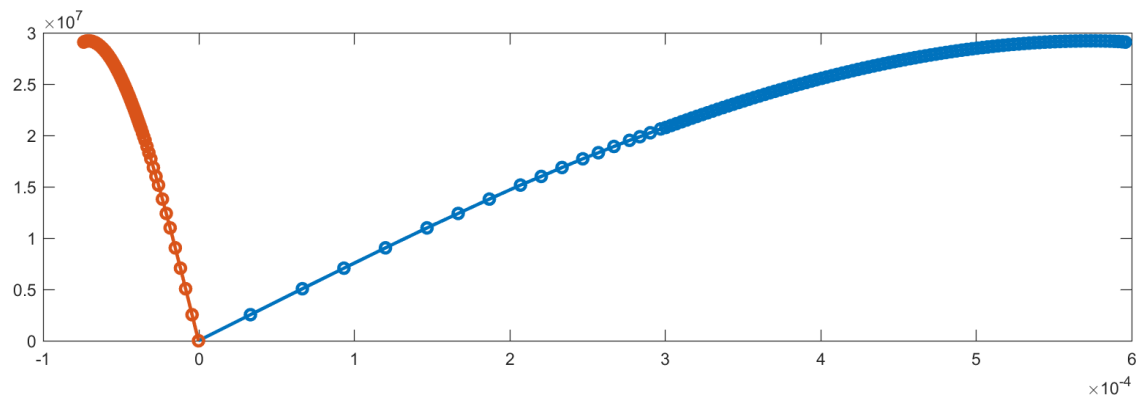

Figure 10. Tensile test (plain plate): $m=0.05 . S_{11}(\mathrm{~Pa})$ versus $G_{11}$ (blue); $S_{11}\left(\mathrm{~Pa}\right.$ ) versus $G_{22}$ (orange); $l=30 \mathrm{~mm} ; m=0.05$; $n=1$. This graphic shows strain-gradient-enabled softening.

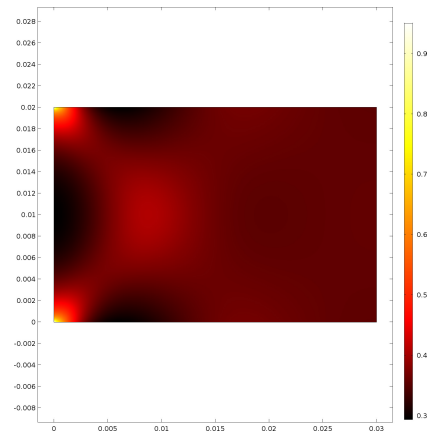

Figure 11. Tensile test (plain plate): $m=0.05$. Color map of $\omega$ at failure point; $l=30 \mathrm{~mm} ; m=0.05 ; n=1$.

Moreover, as is shown in Table $1, m$ is the (nondimensional) weight of secondgradient terms in the internal strain energy. Further, the parameter $n$, introduced in the constitutive assumptions (17), determines the effect of damage on the microstructure; i.e., damage can either affinely magnify $(n>0)$ or affinely shrink $(n<0)$ the square of the characteristic length that is, in millimeters, given by $\sqrt{m}$. Finally, $d$ is the diameter of the circular hole appearing in Figure 7. We make clear that the abscissa of the stress-strain plots which will be shown in the sequel is not equal to the ratio of the imposed displacement $\bar{u}$ to $l$ (i.e., it is not the global strain), but it is in fact the value of $G_{11}$ at the midpoint of the right side of the rectangular domain, i.e., the local strain.

In Figure 8, the stress-strain relationship for the tensile simulation of a plain plate is shown for a first gradient continuum, which means for $m=0$. A slight loss of material stiffness is observed, which however does not lead to any decrease in stress as strain increases. In Figure 9, a contour plot of the damage variable is shown at failure point, in the same example. We remark that the absence of 

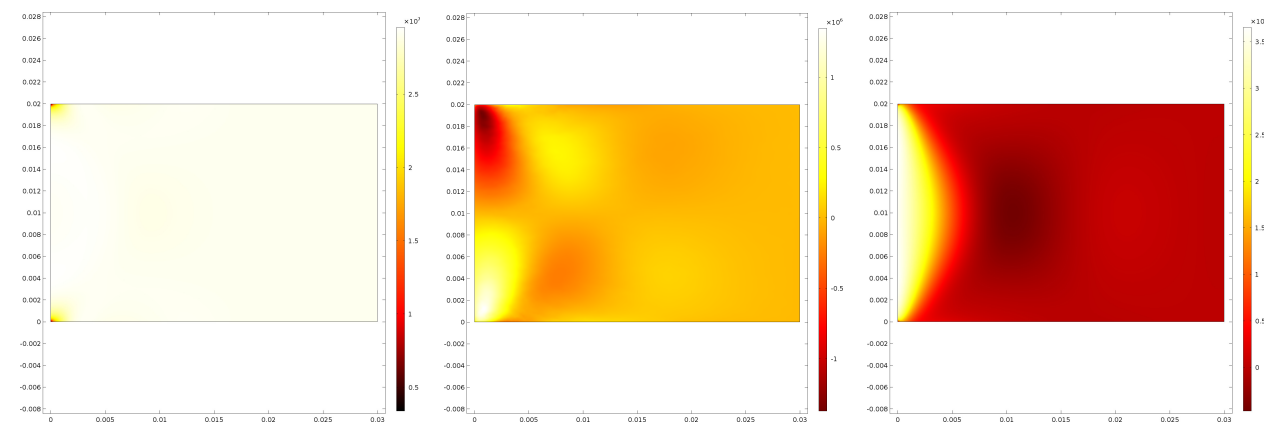

Figure 12. Tensile test (plain plate): $m=0.05$. Color map of $S_{11}(\mathrm{~Pa})$ (left), $S_{12}(\mathrm{~Pa})$ (center), $S_{22}(\mathrm{~Pa})$ (right) at failure point; $l=30 \mathrm{~mm} ; m=0.05 ; n=1$.

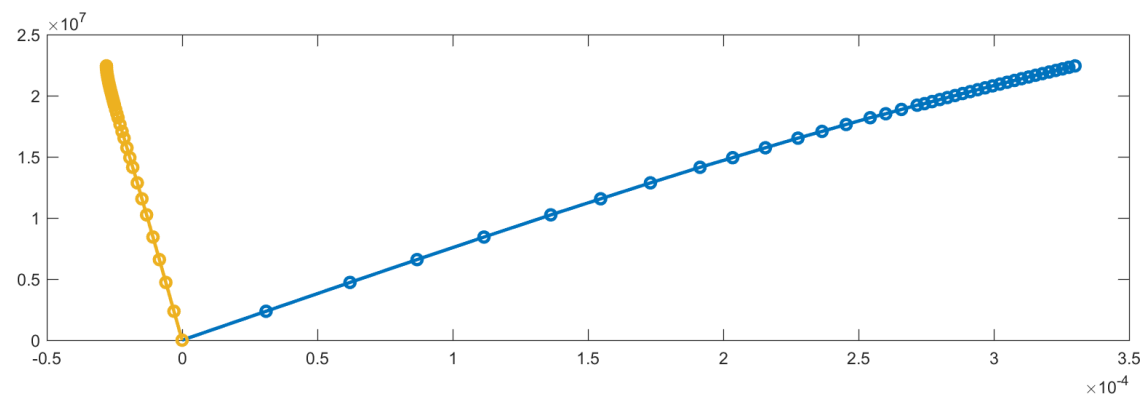

Figure 13. Tensile test (perforated plate): $m=0.05 . S_{11}(\mathrm{~Pa})$ versus $G_{11}$ (blue); $S_{11}(\mathrm{~Pa})$ versus $G_{22}$ (orange); $l=30 \mathrm{~mm} ; m=0.05$; $n=1$. This graphic does not exhibit softening. The value of $m$ required to have softening is relatively much higher.

nonlocal contributions to the internal strain energy density, which would prevent strain localization, results in the absence of any boundary layer and, thus, in a burst of damage in very localized zones. This fact leads to mesh dependency as, whatever the finite element characteristics (size and geometry of mesh and shape function), the first failure is always observed in one single finite element. In Figure 10, which represents the stress-strain diagram for the experiment in Figure 6 and for $m=0.05$, a loss of material stiffness is observed as well. Since the increase of damage due to localization is slowed down by the presence of second-gradient contributions, failure of the material occurs well after that in the case of $m=0$ and a stationary point of the stress-strain relationship is observed for a longitudinal strain corresponding to approximately $G_{11}=5.8 \times 10^{-4}$. In Figure 11, a contour plot of the damage variable is shown at failure point for the same example. We remark that the presence of nonlocal contributions to the internal strain energy density 


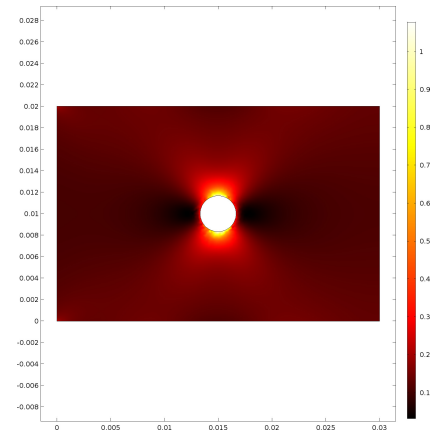

Figure 14. Tensile test (perforated plate): $m=0.05$. Color map of $\omega$ at failure point; $l=30 \mathrm{~mm} ; m=0.05 ; n=1$. This graphic is coherent with well known analytical results in the theory of firstgradient linear homogeneous isotropic elasticity.
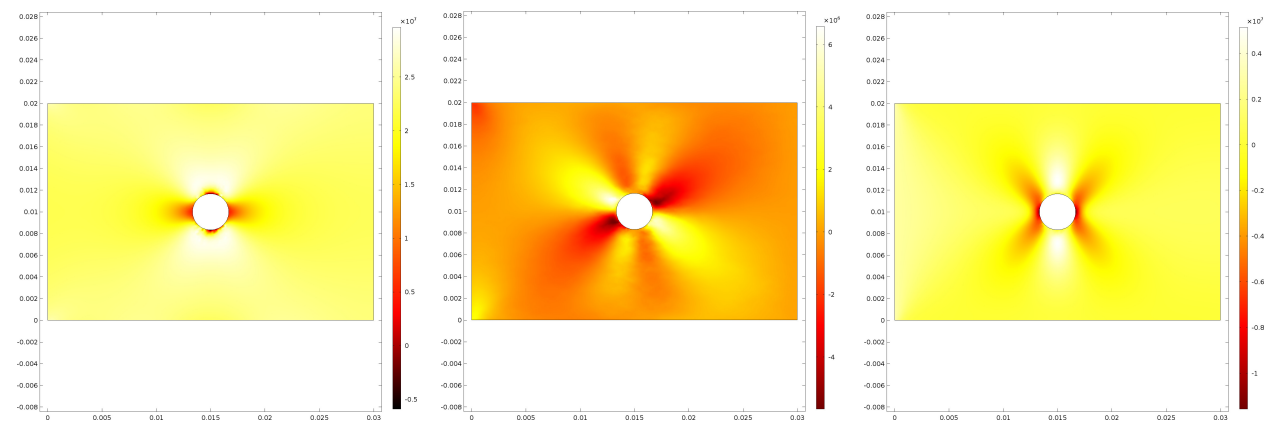

Figure 15. Tensile test (perforated plate): $m=0.05$. Color map of $S_{11}(\mathrm{~Pa})$ (left), $S_{12}(\mathrm{~Pa})$ (center), $S_{22}(\mathrm{~Pa})$ (right) at failure point; $l=30 \mathrm{~mm} ; m=0.05 ; n=1$.

prevents strain localization and mesh dependency is not observed since the regions with the highest value of damage at the left corners of the specimen are larger than the size of a single finite element. In Figure 12 the contour plots at failure point of the components of the stress tensor are shown. Figures 13, 14, and 15 show, respectively, the stress-strain curve, the damage contour plot at failure point, and the contour plots of the components of the stress tensor for the test described in Figure 7, when $m=0.05$. As is clear from Figure 14, failure occurs at the intersection of the transversal axis of the rectangular specimen with the perimeter of the internal circular void and mesh dependency is again avoided by the presence of second-gradient contributions. Still, the characteristic length introduced by means of second gradient is not sufficiently large to limit the increase of damage up to failure and, thus, to see a change of sign in the derivative of the stress-strain 


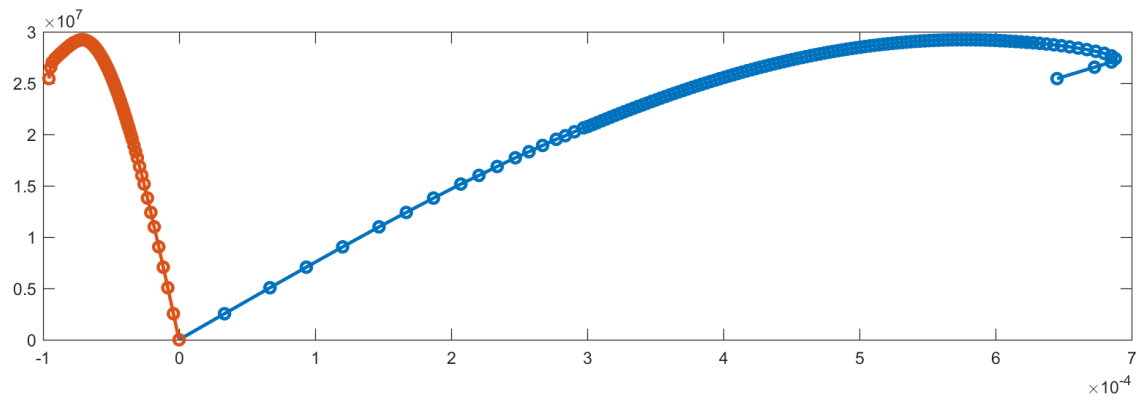

Figure 16. Tensile test (plain plate): $m=0.1 . S_{11}(\mathrm{~Pa})$ versus $G_{11}$ (blue); $S_{11}(\mathrm{~Pa})$ versus $G_{22}$ (orange); $l=30 \mathrm{~mm} ; m=0.1$; $n=1$. This graphic shows a softening behavior and a snap-backlike transition due to localized elastic unloading at the right side of the reference domain.

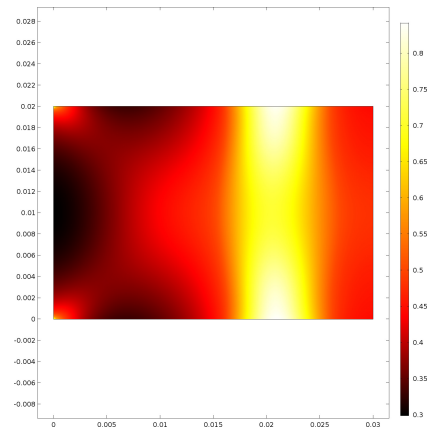

Figure 17. Tensile test (plain plate): $m=0.1$. Color map of $\omega$ at failure point; $l=30 \mathrm{~mm} ; m=0.1 ; n=1$.

relationship. In Figure 16, the stress-strain curve is shown for the test in Figure 6, when $m=0.1$. Clearly, in this case the weight of the second-gradient contribution is sufficiently high to see a decrease of stress as strain increases. It is also relevant that, for such value of $m$, a snap-back like transition, due to elastic unloading of the region of the specimen adjacent to the right side, occurs. Indeed, as shown in Figure 17, failure occurs at a banded region in the middle of the specimen. It is worth remarking that this phenomenon is not a snap-back in the proper sense of the word, because there is no instability involved in the process. In Figure 20 the contour plot of damage at failure point is shown for the same experiment and for a different width of the rectangular specimen, in order to show that the position of the damage band remains unchanged. This evidence is highlighted in Figure 18. In Figure 19 contour plots of the components of the stress tensor are shown. In Figure 21, the contour plot of damage at failure point for the test in Figure 6, 


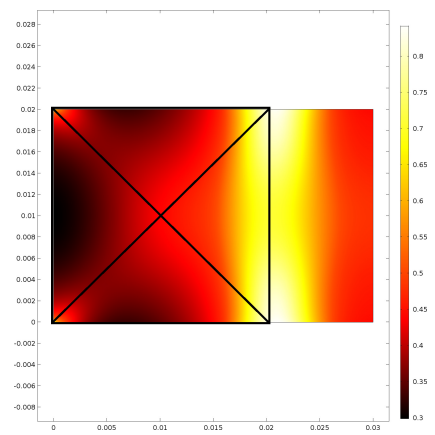

Figure 18. Tensile test (plain plate): $m=0.1$. Color map of $\omega$ at failure point; $l=30 \mathrm{~mm} ; m=0.1 ; n=1$.
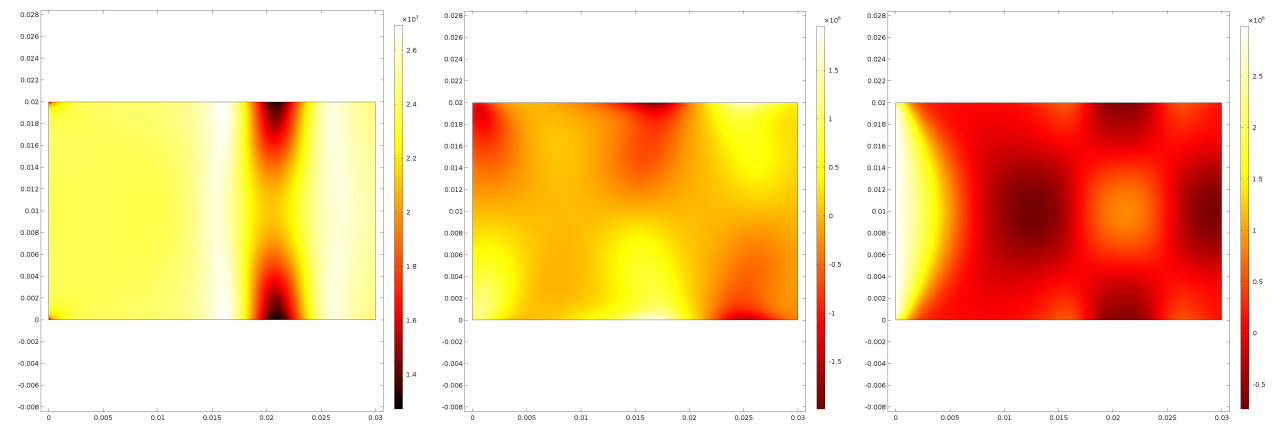

Figure 19. Tensile test (plain plate): $m=0.1$. Color map of $S_{11}(\mathrm{~Pa})$ (left), $S_{12}(\mathrm{~Pa})$ (center), $S_{22}(\mathrm{~Pa})$ (right) at failure point; $l=30 \mathrm{~mm} ; m=0.1 ; n=1$.

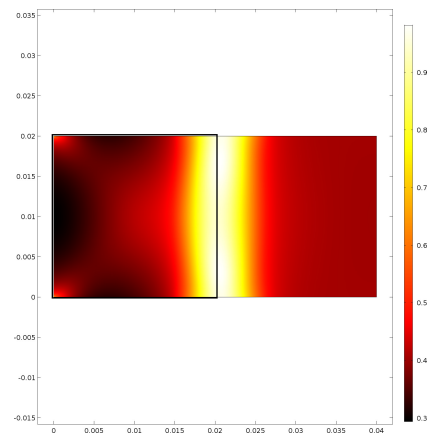

Figure 20. Tensile test (plain plate): $m=0.1$. Color map of $\omega$ at failure point; $l=40 \mathrm{~mm} ; m=0.1 ; n=1$.

when $m=0.1$, is shown for $n=0$, i.e., the second-gradient coefficients (or the microstructure) are not sensitive at all to the internal state of damage. It is clear 


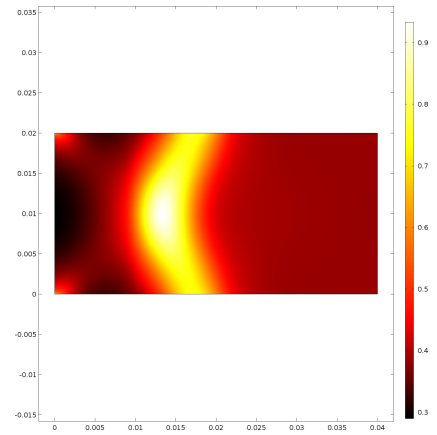

Figure 21. Tensile test (plain plate): $m=0.1$. Color map of $\omega$ at failure point; $l=40 \mathrm{~mm} ; m=0.1 ; n=0$.

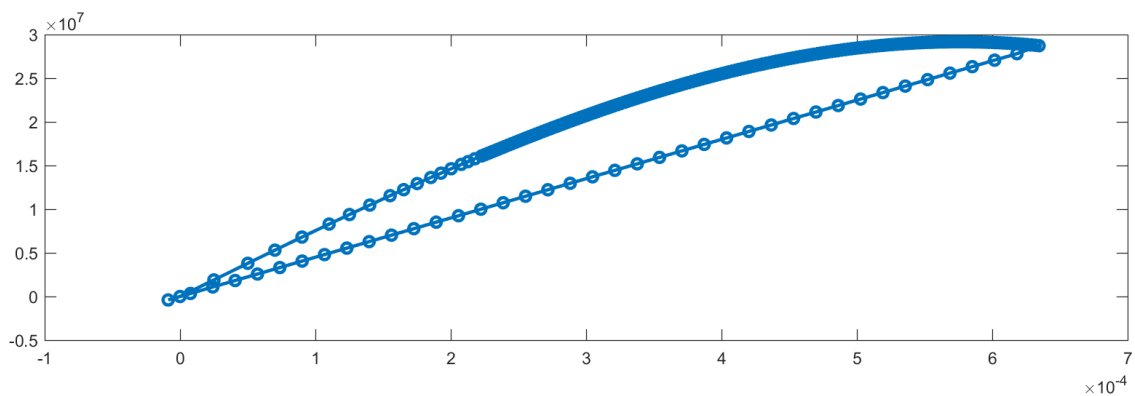

Figure 22. Tensile test (plain plate): $m=0.1 . S_{11}(\mathrm{~Pa})$ versus $G_{11} ; l=30 \mathrm{~mm} ; m=0.1 ; n=1$. This graphic shows dependence of the system upon the path (loading-unloading). The measure of the area inside the cycle is roughly equal to the dissipated energy. After the unloading, the specimen comes back to the initial unstressed configuration. No plastic effect is taken into account.

that the main difference between Figures 17 and 21 is the shape of the damage band. The two figures were obtained under the same conditions, reported in their captions, except for the value of $n$. The damage band is distorted for $n=0$. In Figures 23, 24 , and 25 the stress-strain curve, the damage contour plot at failure point, and the contour plot of the components of the stress tensor for the test described in Figure 6 (with $m=1$ ) are shown, respectively. As is clear from Figure 23, in this case no snap-back-like transition is observed. This is due to the fact that the region of the specimen subject to elastic unloading does not include the point where stress and strain are evaluated in Figure 23, i.e., the middle point of the right side, since, as shown in Figure 24, the damage band touches the right boundary of the specimen. In Figure 22 dependence of the system upon the path is shown in a loading-unloading cycle for the data reported in the caption. Since no plastic effects 


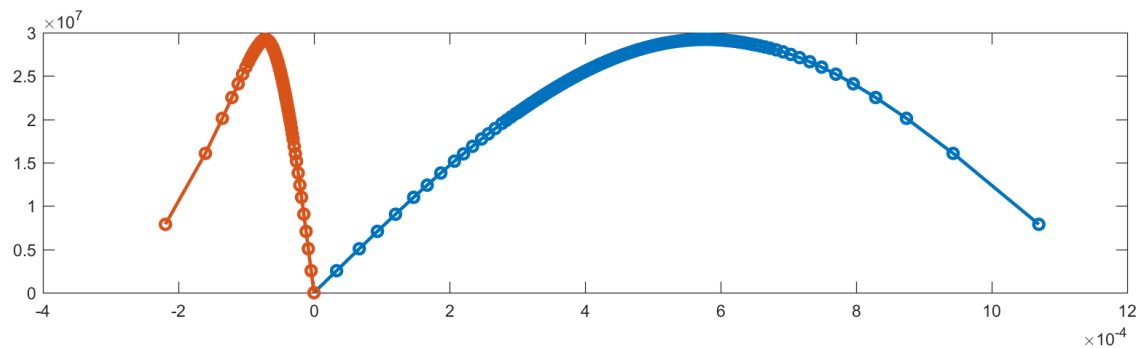

Figure 23. Tensile test (plain plate): $m=1$. $S_{11}(\mathrm{~Pa})$ versus $G_{11}$ (blue); $S_{11}(\mathrm{~Pa})$ versus $G_{22}$ (orange); $l=30 \mathrm{~mm} ; m=1 ; n=1$. This graphic shows strain-gradient-enabled softening.

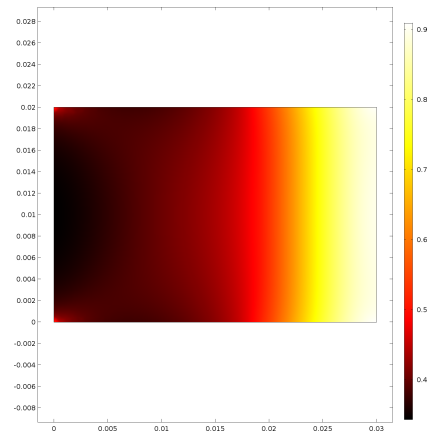

Figure 24. Tensile test (plain plate): $m=1$. Color map of $\omega$ at failure point; $l=30 \mathrm{~mm} ; m=1 ; n=1$.
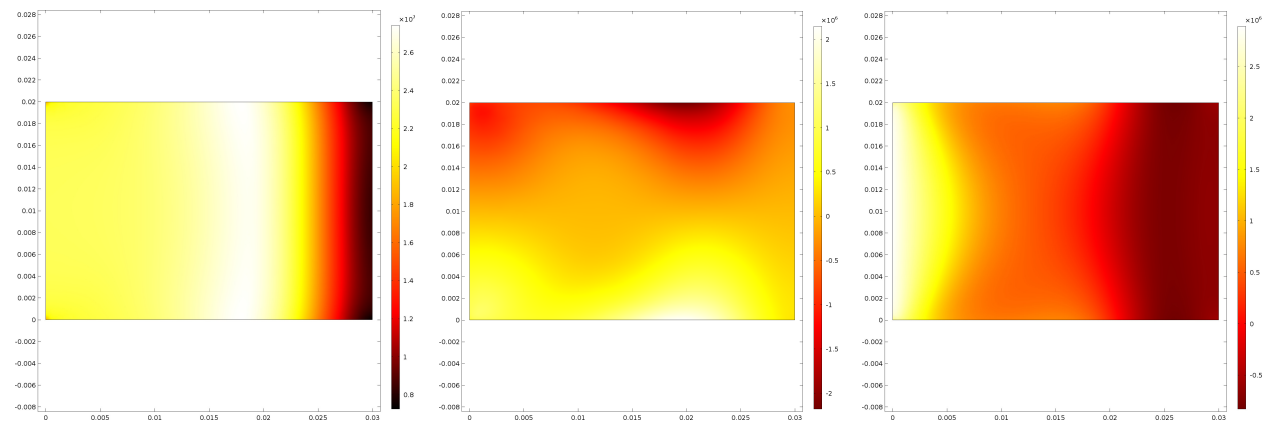

Figure 25. Tensile test (plain plate): $m=1$. Color map of $S_{11}(\mathrm{~Pa})$ (left), $S_{12}(\mathrm{~Pa})$ (center), $S_{22}(\mathrm{~Pa})$ (right) at failure point; $l=30 \mathrm{~mm}$; $m=1 ; n=1$.

are taken into account in the model, after the unloading the specimen comes back to the initial unstressed configuration. Finally, in Figures 26 and 27 mesh-dependency 


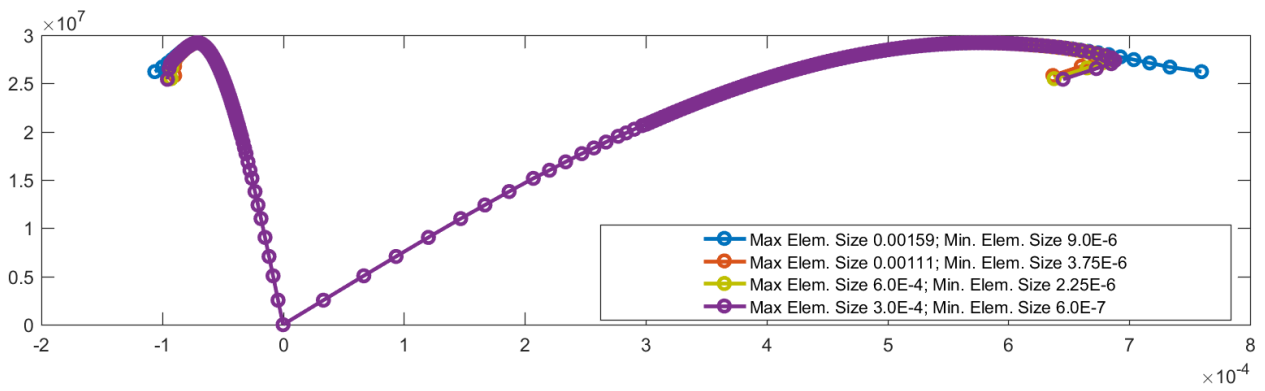

Figure 26. $S_{11}(\mathrm{~Pa})$ versus $G_{11}$ (left); $S_{11}(\mathrm{~Pa})$ versus $G_{22}$ (right); $l=30 \mathrm{~mm} ; m=0.1 ; n=1$. Mesh-dependency study for the tensile test (plain plate). The mesh is triangular and Delaunay-tessellated. Maximum and minimum element sizes are expressed in meters.

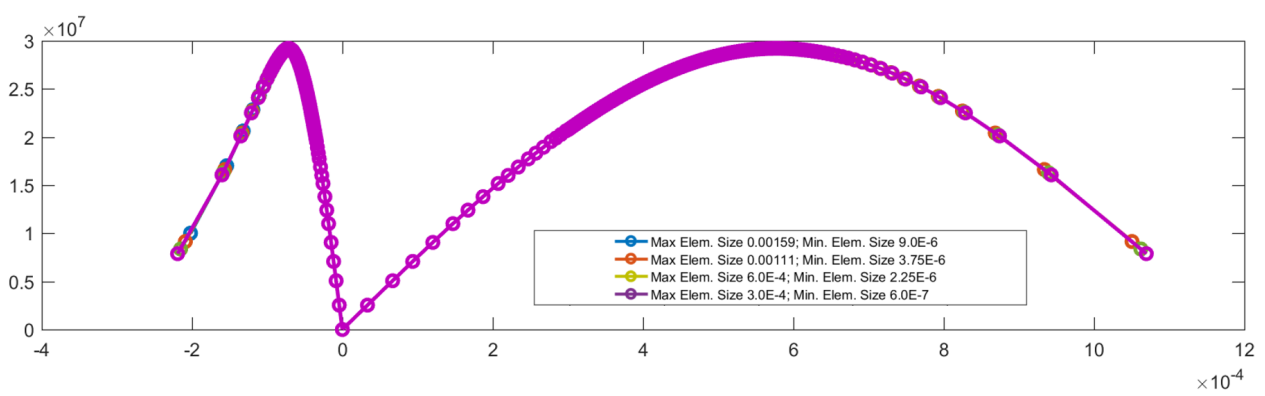

Figure 27. $S_{11}(\mathrm{~Pa})$ versus $G_{11}$ (left); $S_{11}(\mathrm{~Pa})$ versus $G_{22}$ (right); $l=30 \mathrm{~mm} ; m=1 ; n=1$. Mesh-dependency study for the tensile test (plain plate). The mesh is triangular and Delaunay-tessellated. Maximum and minimum element sizes are expressed in meters.

parametric studies, parametrized over the element size, are reported, respectively, for $m=0.1$ and $m=1$, in the case of the test in Figure 6.

The size of the load parameter step $\Delta \bar{u}_{i}$ has been tuned in order to avoid stepdependent simulations. The step size can be higher, still keeping a good accuracy in the numerical solution, when the specimen is in a regime which resembles the elastic one, i.e., when the stress-strain dependence is very close to linear, while damage increase is overestimated to a certain extent when the step size is too large. In that case, the load-parameter step has been decreased accordingly.

\section{Conclusion and outlooks}

Continuum damage mechanics, because of the presence of strain localization, deserves a nonlocal generalization. Advantages and challenges of the incorporation of nonlocal effects, by including either the gradient of damage or the second gradient 
of displacement in the internal strain energy, have been discussed. In this paper we have exploited the case of a damage continuum isotropic two-dimensional strain gradient model. In order to obtain a well posed system of PDEs, BCs, and KKT conditions, a variational approach has been used. Indeed, what is worth remarking is that the variational approach developed in this work allows us to recover not only the relevant governing equations and an incremental damage evolution law, but also boundary conditions which lead to a well posed problem [dell'Isola et al. 2015b; dell'Isola and Placidi 2011; dell'Isola et al. 2015a; Piola 2014]. We have shown that, in the present model, the regularizing effect of the strain gradient terms results, most of the time, in an irreversible softening behavior (i.e., the derivative of stress with respect to strain becomes negative) and, sometimes, even in a kind of snap-back-like transition due to localized elastic unloading. This localized elastic unloading, in the examples that have been shown, clearly arises from the vertically banded damage leading to failure, very likely formed by the propagation and combination of damage from the upper- and lower-left corners. In fact, along this damage band the material is relaxed very much in comparison to other regions of the specimen, i.e., the stiffness, there, is much lower than in other regions. Hence, this region is elongating much more than other regions which, in order to keep the global strain as the one assigned by boundary conditions, are shrunk. We have shown that the position of the damage band depends upon $m$, which weights the second-gradient contribution to the strain energy, and its shape depends upon $n$, which relates such contribution to damage. No snap-back-like transition due to localized elastic unloading at the right side of the reference domain occurs when the damage band touches the right boundary. Simulations were performed also using quadratic Lagrange elements (instead of Hermite cubic elements, for $m>0$ ) and squared meshes (instead of triangular Delaunay-tessellated meshes), confirming the reliability of the results in such a numerically challenging task.

The outlooks of this work are the following. First of all, it would be interesting to exploit the 3D case, in order to understand if geometrical effects due to a higher domain dimensionality could give rise to qualitatively different damage patterns. Then, in order to fit experimental data, it is necessary to develop methods for the experimental and/or theoretical characterization of the constitutive coefficients related to damage. Having a theoretical characterization of such coefficients would be ideal, because it would yield the identification of model parameters related to damage less costly. A promising approach to achieve this goal is to use identification methods based on granular micromechanics [Misra and Singh 2015; 2013], where some kind of damage mechanisms are modeled at the microlevel. Another important line of research that could be potentially pursued starting from this paper would deal with the extension of the present work in order to include plastic phenomena [Contrafatto and Cuomo 2002]. A further numerical campaign has to 
be carried out in order to better investigate the variety of phenomena that can be exhibited by this model. There are a number of research questions which are still open; e.g., does higher $m$ with $0.05<m<0.1$ always imply rightmost damage band? In any case, on the basis of the results shown in this paper, we can formulate several research hypotheses. A better understanding of the simple model studied in this paper could allow significant advancements for the development of a more involved model including, e.g., anisotropy, large deformations, plasticity, different dissipated energy, etc., based upon the same working principle, and that will be the subject of further investigations. We observe that it could be of interest to generalize the results of this paper, e.g., to the modeling of damaging processes in bone tissues [Andreaus et al. 2015; 2014; Giorgio et al. 2016a; 2016b] and of cementitious and granular materials [Misra and Singh 2015; 2013; Yang and Misra 2012; Yang et al. 2011]. Finally, this approach could be especially useful also for the modeling of damage in 2D structures such as pantographic sheets, fiber textile composites, and elastic nets [Spagnuolo et al. 2017; Turco et al. 2017; 2016; dell'Isola et al. 2016; Eremeyev et al. 2017; Battista et al. 2015].

\section{Acknowledgements}

Misra was supported by United States NSF grant CMMI 1727433. This work was also supported by a grant from the Government of the Russian Federation (contract number 14.Y26.31.0031).

\section{References}

[Ambrosio and Tortorelli 1990] L. Ambrosio and V. M. Tortorelli, "Approximation of functionals depending on jumps by elliptic functionals via $\Gamma$-convergence", Comm. Pure Appl. Math. 43:8 (1990), 999-1036.

[Amor et al. 2009] H. Amor, J.-J. Marigo, and C. Maurini, "Regularized formulation of the variational brittle fracture with unilateral contact: numerical experiments", J. Mech. Phys. Solids 57:8 (2009), 1209-1229.

[Andreaus et al. 2014] U. Andreaus, I. Giorgio, and T. Lekszycki, "A 2-D continuum model of a mixture of bone tissue and bio-resorbable material for simulating mass density redistribution under load slowly variable in time", Z. Angew. Math. Mech. 94:12 (2014), 978-1000.

[Andreaus et al. 2015] U. Andreaus, I. Giorgio, and A. Madeo, "Modeling of the interaction between bone tissue and resorbable biomaterial as linear elastic materials with voids", Z. Angew. Math. Phys. 66:1 (2015), 209-237.

[Battista et al. 2015] A. Battista, C. Cardillo, D. Del Vescovo, N. L. Rizzi, and E. Turco, "Frequency shifts induced by large deformations in planar pantographic continua", Nanomech. Sci. Tech. 6:2 (2015), 161-178.

[Bažant 1980] Z. P. Bažant, "Work inequalities for plastic fracturing materials", Int. J. Solids Struct. 16:10 (1980), 873-901.

[Bažant and Jirásek 2002] Z. P. Bažant and M. Jirásek, "Nonlocal integral formulations of plasticity and damage: survey of progress", J. Eng. Mech. 128:11 (2002), 1119-1149. 
[Bažant and Pijaudier-Cabot 1988] Z. P. Bažant and G. Pijaudier-Cabot, "Nonlocal continuum damage, localization instability and convergence”, J. Appl. Mech. 55:2 (1988), 287-293.

[Bourdin et al. 2008] B. Bourdin, G. A. Francfort, and J.-J. Marigo, "The variational approach to fracture", J. Elasticity 91:1-3 (2008), 5-148.

[Comi 1999] C. Comi, "Computational modelling of gradient-enhanced damage in quasi-brittle materials", Mech. Cohesive-Frictional Mat. 4:1 (1999), 17-36.

[Contrafatto and Cuomo 2002] L. Contrafatto and M. Cuomo, "A new thermodynamically consistent continuum model for hardening plasticity coupled with damage”, Int. J. Solids Struct. 39:25 (2002), 6241-6271.

[dell'Isola and Placidi 2011] F. dell' Isola and L. Placidi, "Variational principles are a powerful tool also for formulating field theories", pp. 1-15 in Variational models and methods in solid and fluid mechanics, edited by F. dell'Isola and S. Gavrilyuk, CISM Courses and Lect. 535, Springer, 2011.

[dell'Isola et al. 2015a] F. dell'Isola, U. Andreaus, and L. Placidi, "At the origins and in the vanguard of peridynamics, non-local and higher-gradient continuum mechanics: an underestimated and still topical contribution of Gabrio Piola”, Math. Mech. Solids 20:8 (2015), 887-928.

[dell' Isola et al. 2015b] F. dell'Isola, P. Seppecher, and A. Della Corte, "The postulations à la d'Alembert and à la Cauchy for higher gradient continuum theories are equivalent: a review of existing results", Proc. A. 471:2183 (2015), art. id. 20150415.

[dell' Isola et al. 2016] F. dell'Isola, I. Giorgio, M. Pawlikowski, and N. L. Rizzi, "Large deformations of planar extensible beams and pantographic lattices: heuristic homogenization, experimental and numerical examples of equilibrium", Proc. A 472:2185 (2016), art. id. 20150790.

[Eremeyev et al. 2017] V. A. Eremeyev, F. dell'Isola, C. Boutin, and D. Steigmann, "Linear pantographic sheets: existence and uniqueness of weak solutions", J. Elasticity (online publication November 2017).

[Forest 2009] S. Forest, "Micromorphic approach for gradient elasticity, viscoplasticity, and damage", J. Eng. Mech. 135:3 (2009), 117-131.

[Giorgio et al. 2016a] I. Giorgio, U. Andreaus, and A. Madeo, "The influence of different loads on the remodeling process of a bone and bioresorbable material mixture with voids", Contin. Mech. Thermodyn. 28:1-2 (2016), 21-40.

[Giorgio et al. 2016b] I. Giorgio, U. Andreaus, D. Scerrato, and F. dell'Isola, "A visco-poroelastic model of functional adaptation in bones reconstructed with bio-resorbable materials", Biomech. Model. Mechanobiol. 15:5 (2016), 1325-1343.

[Hill 1948] R. Hill, "A variational principle of maximum plastic work in classical plasticity", Quart. J. Mech. Appl. Math. 1:1 (1948), 18-28.

[Lorentz and Andrieux 2003] E. Lorentz and S. Andrieux, "Analysis of non-local models through energetic formulations", Int. J. Solids Struct. 40:12 (2003), 2905-2936.

[Maier 1970] G. Maier, "A minimum principle for incremental elastoplasticity with non-associated flow laws”, J. Mech. Phys. Solids 18:5 (1970), 319-330.

[Marigo 1989] J. J. Marigo, "Constitutive relations in plasticity, damage and fracture mechanics based on a work property", Nuclear Eng. Des. 114:3 (1989), 249-272.

[Miehe et al. 2016] C. Miehe, F. Aldakheel, and A. Raina, "Phase field modeling of ductile fracture at finite strains: a variational gradient-extended plasticity-damage theory", Int. J. Plasticity 84 (2016), 1-32.

[Misra and Singh 2013] A. Misra and V. Singh, "Micromechanical model for viscoelastic materials undergoing damage", Contin. Mech. Thermodyn. 25:2-4 (2013), 343-358. 
[Misra and Singh 2015] A. Misra and V. Singh, "Thermomechanics-based nonlinear rate-dependent coupled damage-plasticity granular micromechanics model”, Contin. Mech. Thermodyn. 27:4-5 (2015), 787-817.

[Mühlich et al. 2013] U. Mühlich, L. Zybell, G. Hütter, and M. Kuna, "A first-order strain gradient damage model for simulating quasi-brittle failure in porous elastic solids", Arch. Appl. Mech. 83:6 (2013), 955-967.

[Oliver-Leblond et al. 2016] C. Oliver-Leblond, H. Dumontet, and D. Kondo, "A micro-mechanics based strain gradient damage model: formulation and solution for the torsion of a cylindrical bar", Eur. J. Mech. A Solids 56 (2016), 19-30.

[Peerlings et al. 2001] R. H. J. Peerlings, M. G. D. Geers, R. de Borst, and W. A. M. Brekelmans, "A critical comparison of nonlocal and gradient-enhanced softening continua", Int. J. Solids Struct. 38:44-45 (2001), 7723-7746.

[Pham and Marigo 2010a] K. Pham and J.-J. Marigo, “Approche variationnelle de l'endommagement, I: Les concepts fondamentaux", C. R. Mécanique 338:4 (2010), 191-198.

[Pham and Marigo 2010b] K. Pham and J.-J. Marigo, "Approche variationnelle de l'endommagement, II: Les modèles à gradient”, C. R. Mécanique 338:4 (2010), 199-206.

[Pham et al. 2011a] K. Pham, H. Amor, J.-J. Marigo, and C. Maurini, "Gradient damage models and their use to approximate brittle fracture", Int. J. Damage Mech. 20:4 (2011), 618-652.

[Pham et al. 2011b] K. Pham, J.-J. Marigo, and C. Maurini, "The issues of the uniqueness and the stability of the homogeneous response in uniaxial tests with gradient damage models", J. Mech. Phys. Solids 59:6 (2011), 1163-1190.

[Piola 2014] G. Piola, The complete works, I, edited by F. dell'Isola et al., Advanced Structured Materials 38, Springer, 2014.

[Placidi 2015] L. Placidi, "A variational approach for a nonlinear 1-dimensional second gradient continuum damage model”, Contin. Mech. Thermodyn. 27:4-5 (2015), 623-638.

[Placidi 2016] L. Placidi, "A variational approach for a nonlinear one-dimensional damage-elastoplastic second-gradient continuum model”, Contin. Mech. Thermodyn. 28:1-2 (2016), 119-137.

[Placidi and El Dhaba 2017] L. Placidi and A. R. El Dhaba, "Semi-inverse method à la Saint-Venant for two-dimensional linear isotropic homogeneous second-gradient elasticity", Math. Mech. Solids 22:5 (2017), 919-937.

[Placidi et al. 2015] L. Placidi, U. Andreaus, A. Della Corte, and T. Lekszycki, "Gedanken experiments for the determination of two-dimensional linear second gradient elasticity coefficients", $Z$. Angew. Math. Phys. 66:6 (2015), 3699-3725.

[Placidi et al. 2017] L. Placidi, U. Andreaus, and I. Giorgio, "Identification of two-dimensional pantographic structure via a linear D4 orthotropic second gradient elastic model", J. Eng. Math. 103 (2017), 1-21.

[Rahali et al. 2016] Y. Rahali, I. Goda, and J. F. Ganghoffer, "Numerical identification of classical and nonclassical moduli of 3D woven textiles and analysis of scale effects", Compos. Struct. 135 (2016), 122-139.

[Reddy 2011a] B. D. Reddy, "The role of dissipation and defect energy in variational formulations of problems in strain-gradient plasticity, I: Polycrystalline plasticity", Contin. Mech. Thermodyn. 23:6 (2011), 527-549. Erratum in 24:1 (2012), 79.

[Reddy 2011b] B. D. Reddy, "The role of dissipation and defect energy in variational formulations of problems in strain-gradient plasticity, II: Single-crystal plasticity", Contin. Mech. Thermodyn. 23:6 (2011), 551-572. 
[Sicsic and Marigo 2013] P. Sicsic and J.-J. Marigo, "From gradient damage laws to Griffith's theory of crack propagation", J. Elasticity 113:1 (2013), 55-74.

[Spagnuolo et al. 2017] M. Spagnuolo, K. Barcz, A. Pfaff, F. dell'Isola, and P. Franciosi, "Qualitative pivot damage analysis in aluminum printed pantographic sheets: numerics and experiments", Mech. Res. Comm. 83 (2017), 47-52.

[Turco et al. 2016] E. Turco, F. dell'Isola, N. L. Rissi, R. Grygoruk, W. H. Müller, and C. Liebold, "Fiber rupture in sheared planar pantographic sheets: numerical and experimental evidence", Mech. Res. Comm. 76 (2016), 86-90.

[Turco et al. 2017] E. Turco, M. Golaszewski, I. Giorgio, and F. D’Annibale, "Pantographic lattices with non-orthogonal fibres: experiments and their numerical simulations", Comp. B 118 (2017), $1-14$.

[Yang and Misra 2012] Y. Yang and A. Misra, "Micromechanics based second gradient continuum theory for shear band modeling in cohesive granular materials following damage elasticity", Int. J. Solids Struct. 49:18 (2012), 2500-2514.

[Yang et al. 2011] Y. Yang, W. Y. Ching, and A. Misra, "Higher-order continuum theory applied to fracture simulation of nanoscale intergranular glassy film", J. Nanomech. Micromech. 1:2 (2011), $60-71$.

[Zybell et al. 2009] L. Zybell, U. Mühlich, and M. Kuna, "Constitutive equations for porous planestrain gradient elasticity obtained by homogenization”, Arch. Appl. Mech. 79:4 (2009), 359-375.

Received 4 Oct 2017. Accepted 26 Apr 2018.

LUCA PLACIDI: luca.placidi@uninettunouniversity.net

Facoltà di Ingegneria, Università Telematica Internazionale Uninettuno, Roma, Italy

EMILIO BARCHIESI: barchiesiemilio@gmail.com

Dipartimento di Ingegneria Strutturale e Geotecnica, Università degli Studi di Roma "La Sapienza", Roma, Italy

ANIL MisRa: amisra@ku.edu

Civil, Environmental and Architectural Engineering Department, University of Kansas, Lawrence, KS, United States 


\title{
ON JETS, ALMOST SYMMETRIC TENSORS, AND TRACTION HYPER-STRESSES
}

\author{
REUVEN SEGEV AND JĘDRZJ ŚNIATYCKI
}

\begin{abstract}
This paper considers the formulation of higher-order continuum mechanics on differentiable manifolds devoid of any metric or parallelism structure. For generalized velocities modeled as sections of some vector bundle, a variational $k$-th order hyper-stress is an object that acts on jets of generalized velocities to produce power densities. The traction hyper-stress is introduced as an object that induces hyper-traction fields on the boundaries of subbodies. Additional aspects of multilinear algebra relevant to the analysis of these objects are reviewed.
\end{abstract}

\section{Introduction}

The present paper considers the basic mathematical objects in the analysis of hyperstresses for a theory defined on differentiable manifolds. Thus, generalized velocities are represented by sections of a vector bundle. Such a setting encompasses both the Lagrangian and Eulerian points of view of continuum mechanics as well as classical field theories of physics. The base manifold of the vector bundle is interpreted accordingly as either the body manifold, the physical space, or spacetime, respectively. It is recalled that as early as 1957, Walter Noll [1959] defined a body as a differentiable manifold. Further motivation for the general geometric setting of a manifold can be traced back to [Truesdell and Toupin 1960, p.660] (see also [Segev 1994; 2000; 2013]).

As a generalization of the standard introduction of hyper-stresses in higher-order continuum mechanics, the $k$-th order hyper-stress object, the variational hyperstress, is dual to $k$-jets of sections of the vector bundle (see [Segev 2017]). Continuum mechanics on manifolds differs from standard formulations in Euclidean spaces in the following significant sense. In traditional continuum mechanics, the

\section{Communicated by Francesco dell'Isola.}

Both authors are grateful to BIRS for sponsoring the Banff Workshop on Material Evolution, June 11-18, 2017, which lead to this collaboration. Segev's work has been partially supported by the H. Greenhill Chair for Theoretical and Applied Mechanics and the Pearlstone Center for Aeronautical Engineering Studies at Ben-Gurion University.

MSC2010: primary 74A10; secondary 53Z05.

Keywords: high-order continuum mechanics, differential geometry, vector bundle, hyper-stress, traction, multilinear algebra. 
stress tensor plays two roles: it acts on the derivatives of velocity fields to produce power densities and it induces traction fields on boundaries of subbodies. For a theory on manifolds, however, two distinct mathematical objects play these two roles (see [Segev 2002; 2013]). The variational stress acts on the jets of generalized velocity fields to produce power, while the traction stress induces the traction fields on the boundaries of subbodies. While the variational hyper-stress fields have been considered in [Segev 1986; 2017], we propose here a suitable candidate for the role of traction hyper-stress.

This paper is meant to be used as an introduction to the subject, and additional details regarding the properties of symmetric tensors, used extensively in the analysis of jets, are provided in the Appendix. Thus, Section 2 introduces the basic structure, motivates the use of jets of vector fields, and describes their very basic properties. Section 3 introduces variational hyper-stresses and Section 4 introduces traction hyper-stresses and describes the basic properties of what we refer to as "almost symmetric tensors" used to represent them locally. Finally, a short summary is given in Section 5.

\section{Jets}

Jet bundles, see [Saunders 1989] for a comprehensive treatment, serve as the fundamental objects in the formulation of higher-order continuum mechanics on differentiable manifolds. In this section we review the basic constructions associated with jet bundles of a vector bundle. Firstly, however, we motivate the use of jet bundles in higher-order continuum mechanics and classical field theories.

2.1. The fundamental structure. The basic object we consider here is a vector bundle:

$$
\pi: W \rightarrow \mathscr{X}
$$

The object $\mathscr{X}$ is assumed to be a smooth manifold of dimension $n$, that might have a boundary. We will refer to $\mathscr{X}$ as the base manifold. In the context of the Lagrangian point of view of continuum mechanics, $\mathscr{X}$ is interpreted as the body manifold. In the Eulerian point of view of continuum mechanics, $\mathscr{X}$ is interpreted as the physical space manifold, and in modern formulations of classical field theories, $\mathscr{X}$ is interpreted as the space-time manifold.

No additional structure, such as a Riemannian metric, a connection, a parallelism structure, is assumed for the base manifold. This level of generality is in accordance with the reluctance of modern presentations to use a preferred class of reference states (e.g., [Noll 1959]). In particular, if one wishes to consider live tissues in biomechanical studies, it is unlikely that a preferred reference state of the tissue may be pointed out. Thus, there is no class of preferred coordinate systems on $\mathscr{X}$ 
and denoting coordinates by $x^{i}, i=1, \ldots, n$, a coordinate transformation will be denoted by $x^{i^{\prime}}=x^{i^{\prime}}\left(x^{i}\right)$.

Tangent vectors to the manifold $\mathscr{X}$ are viewed as derivatives of the curves $c$ : $[a, b] \rightarrow \mathscr{X}$. (See [Abraham et al. 1988, pp. 157-158 and 479] for the definition of derivative of a curve at the endpoints and its application in the definition of the tangent space to a manifold at a boundary point.) The tangent space to $\mathscr{X}$ at $x$, denoted by $T_{x} \mathscr{X}$, contains all the tangent vectors at $x$ and the tangent bundle $T \mathscr{X}$ is the collection of all tangent vectors at the various points. Given a coordinate system $\left(x^{i}\right)$ and a point $x_{0}$ with coordinates $x_{0}^{j}$, one has coordinate lines, curves of the form $c_{i}:[a, b] \rightarrow \mathscr{X}$, such that their coordinate representation satisfy

$$
x^{j}(t)=x^{j}\left(c_{i}(t)\right)= \begin{cases}x_{0}^{j}, & \text { if } i \neq j, \\ x_{0}^{j}+t, & \text { if } i=j .\end{cases}
$$

The time derivatives of these curves induce tangent vectors denoted by $\partial_{i}=\dot{c}_{i}$. At each point $x$, the vectors $\left\{\partial_{i}\right\}, i=1, \ldots, n$, form a basis of $T_{x} \mathscr{X}$. The corresponding dual basis of the dual vector space, $T_{x}^{* \mathscr{X}}$, is denoted by $\left\{\mathrm{d} x^{i}\right\}$. Thus,

$$
\mathrm{d} x^{i}\left(\partial_{j}\right)=\delta_{j}^{i} .
$$

For each $x \in \mathscr{X}, W_{x}:=\pi^{-1}(x)$ is a vector space that is isomorphic to some fixed $m$-dimensional vector space $\boldsymbol{W}$, although no natural or particular isomorphism is assumed. In particular, for a pair of points $x, y \in \mathscr{X}$, there is no natural isomorphism of $W_{x}$ with $W_{y}$, although both are isomorphic to $\boldsymbol{W}$. The mapping $\pi$ maps all vectors in $W_{x}$ to the point $x$.

Depending on the terminology and context, a vector $w \in W_{x}$ is interpreted either as a virtual velocity/displacement, or as a generalized velocity, or as variation of the field, at the point $x$. It should be mentioned that for the Lagrangian point of view of continuum mechanics on manifolds, the vector bundle $W$ depends on the particular configuration $\kappa$ of the body in space so that $w$ is interpreted as a velocity of the particle $x$ at the point $\kappa(x)$ in space or as a virtual displacement from $\kappa(x)$.

A generalized velocity field is therefore a section, a mapping $w: \mathscr{X} \rightarrow W$ that assigns to each point $x$ a value for its generalized velocity. It follows that $\pi \circ w=$ $\operatorname{Id} \mathscr{x}$, i.e., $\pi(w(x))=x$.

A vector bundle chart, or a coordinate system, will assign to each $w \in W$ a collection of coordinates $\left(x^{i}, w^{\alpha}\right)$, where $x^{i}$ are coordinates for the point $x=\pi(w)$ and $w^{\alpha}, \alpha=1, \ldots, m$, are the components of $w$ relative to some basis $\left\{\boldsymbol{e}_{\alpha}\right\}$ of $W_{x}$. It is assumed that the bases $\left\{\boldsymbol{e}_{\alpha}\right\}$ for the various points $x$ covered by the charts depend on $x$ smoothly. At each point $x$, covered by the charts $\left(x^{i}, w^{\alpha}\right)$ and $\left(x^{i^{\prime}}, w^{\alpha^{\prime}}\right)$, for any $w \in W_{x}$, we must have $w=w^{\alpha} \boldsymbol{e}_{\alpha}=w^{\alpha^{\prime}} \boldsymbol{e}_{\alpha^{\prime}}$ so that there is a matrix $A_{\alpha}^{\alpha^{\prime}}$, depending on $x$, such that $w^{\alpha^{\prime}}=A_{\alpha}^{\alpha^{\prime}} w^{\alpha}$. 
2.2. Why jets. Say $w: \mathscr{X} \rightarrow W$ is a velocity field. The components of $w(x)$ relative to the chart $\left(x^{i}, w^{\alpha}\right)$ are given in terms of $m$ functions $w^{\alpha}\left(x^{i}\right)$. For the chart $\left(x^{i^{\prime}}, w^{\alpha^{\prime}}\right)$, the components are given by the functions $w^{\alpha^{\prime}}\left(x^{i^{\prime}}\right)$ and evidently

$$
w^{\alpha^{\prime}}\left(x^{i^{\prime}}\right)=A_{\alpha}^{\alpha^{\prime}}\left(x^{j}\right) w^{\alpha}\left(x^{j}\right),
$$

where we have indicated explicitly the dependence of the matrix $A_{\alpha}^{\alpha^{\prime}}$ on the point $x$. Differentiating the last identity, using a comma to denote partial derivatives and the summation convention, we obtain

$$
w_{, i^{\prime}}^{\alpha^{\prime}}=A_{\alpha, j}^{\alpha^{\prime}{ }_{, i^{\prime}}} x^{j} w^{\alpha}+A_{\alpha}^{\alpha^{\prime}} w_{, j}^{\alpha} x_{, i^{\prime}}^{j}
$$

This simple relation indicates a fundamental problem. The derivatives $w_{, i^{\prime}}^{\alpha^{\prime}}$ do not depend only on the derivatives $w_{, i}^{\alpha}$; they depend also on the values of $w^{\alpha}$. In other words, while a generalized velocity as a vector field is a well-defined object, the derivative of the generalized velocity is not a well-defined mathematical object (and in particular, as shown in the last equation, the transformation of the partial derivatives may be viewed as an affine transformation). One cannot separate the values of the derivatives from the values of the velocity field in a manner that will be independent of a chart. As an example, we observe that the derivatives may vanish in one coordinate system while they would be different from zero in another. Nevertheless, if we combine the values of the field and the derivatives into a single object, the transformation rules above show that this object - the first jet of the generalized velocity, $j^{1} w$-is well defined. Thus, the first jet of $w$ is represented in the form $\left(x^{i}, w^{\alpha}, w_{, j}^{\alpha}\right)$, or we may write

$$
j^{1} w=w^{\alpha} \boldsymbol{e}_{\alpha}+w_{, i}^{\alpha} d x^{i} \otimes \boldsymbol{e}_{\alpha} .
$$

The collection of 1-jets to the vector bundle $W$ is denoted as $J^{1} W$.

Similarly, we may consider higher-order derivatives of vector fields. In analogy with the case of first derivatives, one realizes that under transformation of coordinates the components of the $k$-th derivatives $w_{, i_{1}^{\prime} \ldots i_{k}}^{\alpha^{\prime}}$ depend on the values of components of all derivatives $w_{, i_{1} \ldots i_{l}}^{\alpha}, 0 \leq l \leq k$, where we identify the zeroth derivative with the value of the function. Thus, the invariant object is the $k$-jet of the velocity field represented under a coordinate system in the form

$$
\begin{aligned}
j^{k} w=w^{\alpha} \boldsymbol{e}_{\alpha} & +w_{, i_{1}}^{\alpha} d x^{i_{1}} \otimes \boldsymbol{e}_{\alpha} \\
& +w_{, i_{1} i_{2}}^{\alpha} d x^{i_{1}} \otimes d x^{i_{2}} \otimes \boldsymbol{e}_{\alpha}+\cdots+w_{, i_{1} \cdots i_{k}}^{\alpha} d x^{i} \otimes \cdots \otimes d x^{i_{k}} \otimes \boldsymbol{e}_{\alpha},
\end{aligned}
$$

or by $\left(x^{i}, w^{\alpha}, w_{, j_{1}}^{\alpha_{1}}, w_{, j_{1} j_{2}}^{\alpha_{2}}, \ldots, w_{, j_{1} \ldots j_{k}}^{\alpha_{k}}\right)$. Formally, a $k$-jet at a point $x \in \mathscr{X}$ is defined as an equivalence class of sections for the equivalence relation by which two sections $w_{1}$ and $w_{2}$ are equivalent if their values at $x$, together with the values of 
the partial derivatives of their local representative under any chart, are equal, respectively. One can show that this definition is independent of the chart chosen (see [Palais 1968; Saunders 1989]). The collection of $k$-jets to $W$ is denoted by $J^{k} W$.

Since higher-order continuum mechanics involves higher-order derivatives of the generalized velocities, we conclude that the terminology of jet bundles provides an appropriate setting for the formulation of such theories.

2.3. Constructions involving jets. Note that each velocity field determines a jet at any given point. Given a chart, the representation of the jet at $x$, determined by the velocity field $w$, is obtained by differentiating the components of $w$ relative to the local coordinates. Any two velocity fields will determine the same $k$-jet at $x$, if their derivatives up to order $k$ are identical.

On the jet bundle, $J^{k} W$ one defines the following mappings. The source map

$$
\pi^{k}: J^{k} W \rightarrow \mathscr{X} \text {, represented by }\left(x^{i}, w^{\alpha}, w_{, j_{1}}^{\alpha_{1}}, w_{, j_{1} j_{2}}^{\alpha_{2}}, \ldots, w_{, j_{1} \ldots j_{k}}^{\alpha_{k}}\right) \mapsto\left(x^{i}\right) \text {, }
$$

assigns to each jet the point in which it is attached. The mapping

$$
\pi_{l}^{k}: J^{k} W \rightarrow J^{l} W, \quad l<k
$$

represented by

$$
\left(x^{i}, w^{\alpha}, w_{, j_{1}}^{\alpha_{1}}, w_{, j_{1} j_{2}}^{\alpha_{2}}, \ldots, w_{, j_{1} \ldots j_{k}}^{\alpha_{k}}\right) \mapsto\left(x^{i}, w^{\alpha}, w_{, j_{1}}^{\alpha_{1}}, w_{, j_{1} j_{2}}^{\alpha_{2}}, \ldots, w_{, j_{1} \ldots j_{l}}^{\alpha_{k}}\right),
$$

assigns to any $k$-jet a jet of a lower order by omitting the derivatives of order higher than $l$. In particular, identifying $J^{0} W$ with $W$, we have

$$
\pi_{0}^{k}: J^{k} W \rightarrow W
$$

which retains only the value of the generalized velocity field itself.

2.4. Symmetric tensors and jets. Henceforth, we will use the notation and ideas introduced in the Appendix to represent, locally, elements of jet bundles. The tensors considered in the Appendix are homogeneous in the sense that they have a definite order, a local representation of a $k$-jet is an element of the symmetric algebra and is represented in general by a collection of symmetric tensors of all orders $l \leq k$. We recall that the representation in (2-7) uses the regular tensor products that are not appropriate base vectors.

The multilinear mappings that represent a jet are not real valued. Rather, they are valued in $\boldsymbol{V}$ - the typical fiber of the vector bundle. We use a local basis $\left\{\boldsymbol{e}_{\alpha}\right\}$ for the vector spaces $W_{x}$ so that a section of $W$ is locally of the form

$$
w=w^{\alpha} \boldsymbol{e}_{\alpha}
$$

where the components $w^{\alpha}$ are real valued functions. This does not affect the symmetry properties considered above. The basic vector space on which the tensors 
are defined at each point is the tangent space of the manifold at that point. Given a chart with coordinates $\left(x^{i}\right)$, the base vectors induced are $\left\{\partial_{i}\right\}$ and they replace the base vectors $\left\{\boldsymbol{e}_{i}\right\}$ used in the Appendix. The various derivatives in $w_{, \boldsymbol{I}}^{\alpha}$ are covariant tensors and are represented using the dual basis $\left\{\mathrm{d} x^{i}\right\}$. The derivatives $w_{, \boldsymbol{I}}^{\alpha}(x),|\boldsymbol{I}|=l$, are elements of

$$
L_{S}^{l}\left(T_{x} \mathscr{X}, W_{x}\right) \simeq \bigodot^{l} T_{x}^{*} \mathscr{X} \otimes W_{x} .
$$

Thus, we may rewrite now (2-7) in the form

$$
j^{k} w=w_{, I}^{\alpha} \overleftarrow{\mathrm{d} x}^{(\boldsymbol{I})} \otimes \boldsymbol{e}_{\alpha}=\overleftarrow{w}_{, \boldsymbol{I}}^{\alpha} \mathrm{d} x^{(\boldsymbol{I})} \otimes \boldsymbol{e}_{\alpha}, \quad 0 \leq|\boldsymbol{I}| \leq k
$$

An element $A \in J^{k} W$ of the jet bundle with $\pi^{k}(A)=x \in \mathscr{X}$ is of the form

$$
A=j_{x}^{k} w:=\left(j^{k} w\right)(x),
$$

for some section $w$ which may be represented locally as

$$
A=w_{, \boldsymbol{I}}^{\alpha}(x) \overleftarrow{\mathrm{d} x}(\boldsymbol{I}) \otimes \boldsymbol{e}_{\alpha}
$$

Noting that the values of the various $w_{, I}^{\alpha}(x)$ are not constrained by compatibility, any element of the jet bundle may be represented in the form

$$
A=A_{\boldsymbol{I}}^{\alpha} \overleftarrow{\mathrm{d} x}^{(\boldsymbol{I})} \otimes \boldsymbol{e}_{\alpha}=\overleftarrow{A}_{\boldsymbol{I}}^{\alpha} \mathrm{d} x^{(\boldsymbol{I})} \otimes \boldsymbol{e}_{\alpha}, \quad 0 \leq|\boldsymbol{I}| \leq k
$$

$A_{\boldsymbol{I}}^{\alpha} \in \bigodot^{|\boldsymbol{I}|} T_{x}^{*} \mathscr{L} \otimes W_{x}$. Given an element of the jet bundle, one can construct a local section, representing it by using the corresponding Taylor polynomial in any chart.

We finally remark that the representation using $\overleftarrow{\mathrm{d} x}^{(\boldsymbol{I})}$ seems preferable because the components of the jet are exactly the derivatives.

2.5. Duality for jets . In view of (A-67), the dual basis of $\left\{\overleftarrow{\mathrm{d} x}^{(\boldsymbol{I})}|0 \leq| \boldsymbol{I} \mid \leq k\right\}$ is $\left\{\partial_{(\boldsymbol{I})}|0 \leq| \boldsymbol{I} \mid \leq k\right\}$. Note that $\partial_{(\boldsymbol{I})}:=\partial_{i_{1}} \odot \cdots \odot \partial_{i_{|\boldsymbol{I}|}}$ is the symmetrized tensor product while $\partial_{\boldsymbol{I}}$ is the differential operator which is symmetric automatically. Real valued linear mappings on the space of jets at a point $x \in \mathscr{X}$ make up the dual space $\left(J_{x}^{k} W\right)^{*}$. Such a linear functional

$$
\varphi: J_{x}^{k} W \rightarrow \mathbb{R}
$$

is locally of the form

$$
\varphi=\varphi_{\alpha}^{\boldsymbol{I}} \partial_{(\boldsymbol{I})} \otimes \boldsymbol{e}^{\alpha},
$$

so that for $\varphi \in\left(J_{x}^{k} W\right)^{*}, A=j^{k} w(x) \in J_{x}^{k} W$,

$$
\varphi(A)=\varphi_{\alpha}^{\boldsymbol{I}} A_{\boldsymbol{I}}^{\alpha}=\varphi_{\alpha}^{\boldsymbol{I}} w_{, \boldsymbol{I}}^{\alpha},
$$

where $0 \leq|\boldsymbol{I}| \leq k$, unless indicated otherwise. 


\section{Variational hyper-stresses}

In accordance with the variational approach to higher-order continuum mechanics, we view variational hyper-stresses as fields that act on the derivatives of the virtual velocities to produce power densities (see [Segev 2017]). Thus, in the current setting, a variational hyper-stress object should act linearly on the $k$-jet of a field $w$ to produce a density on $\mathscr{X}$.

We recall that for integration over an $n$-dimensional manifold, such as $\mathscr{X}$, densities (integrands) are $n$-forms - alternating (completely antisymmetric) tensor fields of order $n$. The space of $r$-alternating tensors over $T_{x} \mathscr{L}$ will be denoted by $\wedge^{r} T_{x}^{*} \mathscr{X}$ and the bundle of alternating tensors is $\bigwedge^{r} T^{*} \mathscr{X}$. A local coordinate system $\left(x^{i}\right)$ induces such an $n$-form

$$
\mathrm{d} x=\mathrm{d} x^{1} \wedge \cdots \wedge \mathrm{d} x^{n},
$$

where a wedge denotes the exterior product — the antisymmetrized tensor product. Note that antisymmetric tensors cannot have repeated indices and so the multiindices representing base vectors and components are strictly increasing rather than nondecreasing. This implies that $\bigwedge^{n} T_{x}^{*} \mathscr{X}$ is one-dimensional, and $\mathrm{d} x$, the $n$-form induced by a local coordinate system, may serve as a basis. Thus, every $n$-form may be written locally as

$$
\theta=\vartheta(x) \mathrm{d} x
$$

for some real valued function $\vartheta$.

In view of these observations, a variational hyper-stress object at $x$ should be a linear mapping

$$
S_{x}: J_{x}^{k} W \rightarrow \bigwedge^{r} T_{x}^{*} \mathscr{X}
$$

so that $S_{x}\left(j^{k} w(x)\right)$ is the power density. Denoting the bundle of linear mappings $J^{k} W \rightarrow \bigwedge^{n} T^{* \mathscr{X}}$ by $L\left(J^{k} W, \bigwedge^{n} T^{*} \mathscr{X}\right)$,

$$
S_{x} \in L\left(J_{x}^{k} W, \bigwedge^{n} T_{x}^{*} \mathscr{X}\right)=L\left(J^{k} W, \bigwedge^{n} T^{*} \mathscr{X}\right)_{x} .
$$

It is also observed that

$$
L\left(J_{x}^{k} W, \bigwedge^{n} T_{x}^{*} \mathscr{X}\right)=\left(J_{x}^{k} W\right)^{*} \otimes \bigwedge^{n} T_{x}^{*} \mathscr{X},
$$

and

$$
L\left(J^{k} W, \bigwedge^{n} T^{*} \mathscr{X}\right)=\left(J^{k} W\right)^{*} \otimes_{\mathscr{X}} \bigwedge^{n} T^{*} \mathscr{X} .
$$

We conclude that a variational hyper-stress field is a section $S$ of $L\left(J^{k} W\right.$, $\left.\bigwedge^{n} T^{*} \mathscr{X}\right)$. In view of the representation of elements of the dual to the jet bundle in Section 2.5, the local representation of $S$ is of the form

$$
S=S_{\alpha}^{\boldsymbol{I}} \partial_{(\boldsymbol{I})} \otimes \boldsymbol{e}^{\alpha} \otimes \mathrm{d} x .
$$


The action of a variational hyper-stress on the jet of a generalized velocity is the density given by

$$
S\left(j^{k} w\right)=S_{\alpha}^{\boldsymbol{I}} w_{, \boldsymbol{I}}^{\alpha} \mathrm{d} x
$$

and the total power is

$$
P=\int_{\mathscr{C}} S \cdot j^{k} w
$$

where $S \cdot j^{k} w$ is the $n$-form $\left(S \cdot j^{k} w\right)(x)=S(x)\left(j^{k} w(x)\right)$.

\section{Traction hyper-stresses and almost symmetric tensors}

The stress object in traditional continuum mechanics plays two roles. On the one hand, from the variational point of view, the stress object acts on the derivative of the velocity field to produce power. The generalization of this object is the variational hyper-stress introduced above. On the other hand, as a result of Cauchy's stress theorem, the stress object determines the traction field on the boundary of the body and its subbodies. While the same mathematical object plays these two roles in the traditional formulation, in the case of a formulation on manifolds, the traction is determined by a different mathematical object - the traction stress (see [Segev 2013]).

4.1. Traction and traction stresses. For the case $k=1$ - first order continuum mechanics - the traction field on the boundary of $\mathscr{X}$, or in general, any of its subbodies (subregions) $\mathscr{R}$, acts linearly on the values of the generalized velocity $w$ to produce a power density over the boundary, the flux of power. Since the boundaries are manifolds of dimensions $n-1$, a power density over the boundary $\partial \mathscr{R}$ is an $(n-1)$-form over $\partial \mathscr{R}$, that is, a section of $\bigwedge^{n-1} T^{*} \partial \mathscr{R}$. Thus, the traction field on the boundary is a section of

$$
L\left(W, \bigwedge^{n-1} T^{*} \partial \mathscr{R}\right),
$$

where, with some abuse of notation, we have omitted the indication that we restrict $W$ to $\partial \mathscr{R}$. It is observed that the fibers of $\bigwedge^{n-1} T^{*} \partial \mathscr{R}$ are one-dimensional.

A traction stress - an object that unlike a traction field is defined over the entire $\mathscr{X}$ - should induce a traction field on the boundary of each subregion using a generalization of Cauchy's formula. A natural candidate for such a mathematical object is suggested by the following observation. While the space of $(n-1)-$ alternating tensors over $\partial \mathscr{R}$ is one-dimensional, the space $\wedge^{n-1} T^{*} \mathscr{L}$ of $(n-1)$ alternating tensors over $\mathscr{X}$ is $n$-dimensional. While an element of $\wedge^{n-1} T^{*} \mathscr{X}$ assigns a value to any collection of $n-1$ vectors, an element of $\wedge^{n-1} T^{*} \partial \mathscr{R}$ assigns values only to vectors tangent to $\partial \mathscr{R}$. In fact, an element of $\bigwedge^{n-1} T^{*} \mathscr{L}$ may be restricted to act on vectors tangent to $\partial \mathscr{R}$ for every subbody $\mathscr{R}$. Thus, for each subbody $\mathscr{R}$, 
we have a restriction mapping

$$
\rho_{\partial \mathscr{R}}: \bigwedge^{n-1} T^{* \mathscr{X}} \rightarrow \bigwedge^{n-1} T^{*} \partial \mathscr{R}
$$

naturally defined by

$$
\rho_{\partial \mathscr{R}}(\omega)\left(v_{1}, \ldots, v_{n}\right)=\omega\left(v_{1}, \ldots, v_{n}\right), \quad v_{r} \in T \partial \mathscr{R} .
$$

Thus, a traction stress is defined to be an element

$$
\sigma_{0} \in L\left(W, \bigwedge^{n-1} T^{*} \mathscr{X}\right) .
$$

Given a traction stress $\sigma_{0}$, at a point $x$, for any subbody $\mathscr{R}$ with $x \in \partial \mathscr{R}$, a traction $\boldsymbol{t}_{0} \in L\left(W, \bigwedge^{n-1} T^{*} \partial \mathscr{R}\right)$ is determined at $x$ by setting

$$
\boldsymbol{t}_{0}=\hat{\rho}_{\partial \mathscr{R}}(\sigma)=\rho_{\partial \mathscr{R}} \circ \sigma, \quad \text { i.e., } \quad \boldsymbol{t}_{0}(w)=\rho_{\partial \mathscr{R}}(\sigma(w)) .
$$

The last equation is the required generalization of Cauchy's formula to the setting of differentiable manifolds. In analogy with the classical Cauchy theorem, it can be shown that if the traction is given on the boundary of every subbody $\mathscr{R}$, with $x \in \partial \mathscr{R}$, then, assuming that certain consistency conditions hold, a unique traction stress is determined at $x$ (see [Segev and Rodnay 1999; Segev 2013] for details).

A traction stress field is a section of the bundle $L\left(W, \bigwedge^{n-1} T^{*} \mathscr{X}\right)$.

4.2. On the local representation of $(n-1)$-forms and traction stresses. Traction stresses are elements of

$$
L\left(W, \bigwedge^{n-1} T^{*} \mathscr{X}\right) \simeq W^{*} \otimes \bigwedge^{n-1} T^{*} \mathscr{X} .
$$

Thus, we make a few comments on the representation of $(n-1)$-alternating tensors, i.e., for a vector space $\boldsymbol{V}$, we consider elements of $\bigwedge^{n-1} \boldsymbol{V}^{*}$.

We first recall that $\wedge^{n} \boldsymbol{V}^{*}$ is one-dimensional and that $\bigwedge^{n-1} \boldsymbol{V}^{*}$ is $n$-dimensional. Let $\lrcorner$ denote the contraction (inner product) whereby for an alternating $r$-tensor $\omega \in \wedge^{r} \boldsymbol{V}^{*}$ and a vector $\left.v_{1} \in \boldsymbol{V}, v_{1}\right\lrcorner \omega$ is the alternating $(r-1)$-tensor such that

$$
\left.v_{1}\right\lrcorner \omega\left(v_{2}, \ldots, v_{r}\right)=\omega\left(v_{1}, \ldots, v_{r}\right) .
$$

In fact, considering the particular case $r=n-1$, one can view the contraction as a mapping

$$
\left.\widehat{\lrcorner}: \boldsymbol{V} \times \bigwedge^{n} \boldsymbol{V}^{*} \rightarrow \bigwedge^{n-1} \boldsymbol{V}^{*}, \quad \widehat{\lrcorner}(v, \theta)=v\right\lrcorner \theta .
$$

We observe that the definition of the contraction mapping implies immediately that the mapping $\widehat{\lrcorner}$ is bilinear. It follows from the universal property of tensor products that there is a linear mapping, which we still denote as $\widehat{\lrcorner}$, such that

$$
\left.\widehat{\lrcorner}: \boldsymbol{V} \otimes \bigwedge^{n} \boldsymbol{V}^{*} \rightarrow \bigwedge^{n-1} \boldsymbol{V}^{*}, \quad \widehat{\lrcorner}(v \otimes \theta)=v\right\lrcorner \theta .
$$


One can verify that this mapping is injective (e.g., [Segev 2013]), and as the dimensions match, it follows that $\widehat{\lrcorner}$ defines a natural isomorphism

$$
\boldsymbol{V} \otimes \bigwedge^{n} \boldsymbol{V}^{*} \simeq \bigwedge^{n-1} \boldsymbol{V}^{*}
$$

Furthermore, for a basis $\left\{\boldsymbol{e}_{i}\right\}$, a natural basis of $\wedge^{n} \boldsymbol{V}^{*}$ is $\boldsymbol{e}^{1} \wedge \cdots \wedge \boldsymbol{e}^{n}$, and so

$$
\left.\left\{\boldsymbol{e}_{i}\right\lrcorner\left(\boldsymbol{e}^{1} \wedge \cdots \wedge \boldsymbol{e}^{n}\right)\right\}, \quad i=1, \ldots, n,
$$

may serve as a natural basis to $\wedge^{n-1} \boldsymbol{V}^{*}$.

Going back to traction stresses, it follows from the foregoing discussion that

$$
L\left(W, \bigwedge^{n-1} T^{*} \mathscr{X}\right) \simeq W^{*} \otimes \bigwedge^{n-1} T^{*} \mathscr{X} \simeq W^{*} \otimes T \mathscr{X} \otimes \bigwedge^{n} T^{*} \mathscr{X} .
$$

For a given coordinate system $\left(x^{i}\right)$, the collection $\left.\left\{\partial_{i}\right\lrcorner \mathrm{d} x\right\}$ may serve as a basis for $\left(\wedge^{n-1} T^{*} \mathscr{X}\right)_{x}$. As a result, any $\omega$ may be represented locally in the form

$$
\left.\omega=\omega^{i} \partial_{i}\right\lrcorner \mathrm{d} x,
$$

where $\mathrm{d} x$ is defined in (3-1). The local representation of a traction stress will be

$$
\left.\sigma=\sigma_{\alpha}^{i} \boldsymbol{e}^{\alpha} \otimes\left(\partial_{i}\right\lrcorner \mathrm{d} x\right)
$$

and

$$
\left.\sigma(w)=\sigma_{\alpha}^{i} w^{\alpha}\left(\partial_{i}\right\lrcorner \mathrm{d} x\right) .
$$

4.3. Hyper-traction and traction hyper-stresses. By analogy with the case $k=1$ described above, where the traction object acts on the $k-1=0$-jet of the generalized velocity, we propose that a hyper-traction on the boundary $\partial \mathscr{R}$ of a subbody $\mathscr{R}$, be defined as an element

$$
\boldsymbol{t} \in L\left(J^{k-1} W, \bigwedge^{n-1} T^{*} \partial \mathscr{R}\right) \simeq\left(J^{k-1} W\right)^{*} \otimes \bigwedge^{n-1} T^{*} \partial \mathscr{R} .
$$

Thus, the total power flux is given by

$$
\int_{\partial \mathscr{R}} \boldsymbol{t} \cdot j^{k-1} w
$$

A traction hyper-stress field is defined in analogy with the definition of a traction stress, in the sense that it acts on a lower order jet to give an $(n-1)$-form which can be integrated on the boundaries of subbodies. Thus, a traction hyper-stress is defined to be an element

$$
\sigma_{0} \in L\left(J^{k-1} W, \bigwedge^{n-1} T^{*} \mathscr{X}\right) \simeq\left(J^{k-1} W\right)^{*} \otimes T \mathscr{X} \otimes \bigwedge^{n} T^{*} \mathscr{X} .
$$

It follows from the foregoing analysis that a traction hyper-stress is represented locally in the form

$$
\left.\sigma_{0}=\sigma_{\alpha}^{J j} \partial_{(\boldsymbol{J})} \otimes \boldsymbol{e}^{\alpha} \otimes\left(\partial_{i}\right\lrcorner \mathrm{d} x\right), \quad 0 \leq|\boldsymbol{J}| \leq k-1 .
$$


A traction hyper-stress field is a section of $L\left(J^{k-1} W, \bigwedge^{n-1} T^{*} \mathscr{X}\right)$ and the action of a hyper-stress field $\sigma$ on the $(k-1)$-jet of a generalized velocity $w$ is given by

$$
\left.\sigma \cdot j^{k-1} w=\sigma_{\alpha}^{J j} w_{, J}^{\alpha} \partial_{i}\right\lrcorner \mathrm{d} x .
$$

These natural extensions imply that the Cauchy formula (4-5) remains applicable as it simply represents the restriction of forms. Thus, given a traction hyper-stress field $\sigma$ and a generalized velocity field $w$, the total flux of power through the boundary $\partial \mathscr{R}$ is

$$
\int_{\partial \mathscr{R}} \boldsymbol{t} \cdot j^{k-1} w=\int_{\partial \mathscr{R}} \hat{\rho}_{\partial \mathscr{R}}(\sigma) \cdot j^{k-1} w .
$$

It is emphasized that the array $\sigma_{\alpha}^{J j}$ representing a traction hyper-stress is symmetric with respect to permutations of the multi-index $\boldsymbol{J}$ and for this reason it appears in conjunction with the symmetrized basis $\partial_{(J)}$. In particular, no symmetry is expected for permutations that "mix" the indices $\boldsymbol{J}$ and $j$. Thus, for a fixed value $l=|\boldsymbol{J}|$, we refer to the tensor $\sigma_{\alpha}^{J j}$ as the almost symmetric tensor.

4.4. Almost symmetric tensors. In order to simplify the notation we will consider henceforth only real valued almost symmetric tensors. That is, for some given vector space $\boldsymbol{V}$ we consider elements of $\left(\bigodot^{l-1} \boldsymbol{V}\right) \otimes \boldsymbol{V}$ rather than elements of $\left(\bigodot^{l-1} \boldsymbol{V}\right) \otimes \boldsymbol{V}^{*} \otimes \boldsymbol{V} \otimes \bigwedge^{n} \boldsymbol{V}^{*}$

Let $\left\{\boldsymbol{e}_{i}\right\}$ be a basis in $\boldsymbol{V}$. Then, we may use either $\left\{\boldsymbol{e}_{(\boldsymbol{J})}\right\}, 0 \leq|\boldsymbol{J}| \leq l-1$, or the basis $\left\{\overleftarrow{\boldsymbol{e}}_{(\boldsymbol{J})}\right\}$ for $\bigodot^{l-1} \boldsymbol{V}$ in analogy with (A-56). A real valued almost symmetric tensor $T$ can be represented in the form

$$
T=T^{I} \boldsymbol{e}_{I}=T^{\boldsymbol{J} j} \overleftarrow{\boldsymbol{e}}_{(\boldsymbol{J})} \otimes \boldsymbol{e}_{j}=\overleftarrow{T}^{\boldsymbol{J} j} \boldsymbol{e}_{(\boldsymbol{J})} \otimes \boldsymbol{e}_{j}
$$

where $0 \leq|\boldsymbol{J}| \leq l-1,0 \leq|I| \leq l$, and

$$
\overleftarrow{\boldsymbol{e}}_{(\boldsymbol{J})}=\frac{(l-1) !}{\boldsymbol{J} !} \boldsymbol{e}_{(\boldsymbol{J})}, \quad \overleftarrow{T}^{\boldsymbol{J}}=\frac{(l-1) !}{\boldsymbol{J} !} T^{J}
$$

For the dual space we have

$$
\left[\left(\bigodot^{l-1} \boldsymbol{V}\right) \otimes \boldsymbol{V}\right]^{*} \simeq\left(\bigodot^{l-1} \boldsymbol{V}^{*}\right) \otimes \boldsymbol{V}^{*}
$$

so that its elements may be referred to as almost symmetric cotensors. For the basis $\left\{\overleftarrow{\boldsymbol{e}}_{(\boldsymbol{J})} \otimes \boldsymbol{e}_{j}\right\}$, the dual basis will be $\left\{\boldsymbol{e}^{(\boldsymbol{J})} \otimes \boldsymbol{e}^{j}\right\}$. An element $\varphi$ of $\left[\left(\bigodot^{l-1} \boldsymbol{V}\right) \otimes \boldsymbol{V}\right]^{*}$ is represented in the form

$$
\varphi=\varphi_{\boldsymbol{J} j} \boldsymbol{e}^{(\boldsymbol{J})} \otimes \boldsymbol{e}^{j}
$$

with

$$
\varphi(T)=\varphi_{\boldsymbol{J} j} T^{\boldsymbol{J} j} .
$$




\section{Conclusion}

We have reviewed above the language needed for the formulation of higher-order continuum mechanics on differentiable manifolds. In particular, we have proposed the mathematical object that we believe should play the role of traction hyper-stress. While for the case $k=1$, the traction stress has been defined in [Segev 2002; 2013], no natural analogous definition has been presented in [Segev 2017]. In fact, in [Segev 2017] some of the difficulties have been indicated and subsequently avoided by using iterated jet bundles (the jet bundle of the jet bundle) and the corresponding dual objects rather than analyzing directly higher jet bundles and hyper-stresses.

Nevertheless, no relation between variational hyper-stresses and the proposed traction hyper-stresses has been given above. We hope to study this relation in a forthcoming work.

\section{Appendix: Notes on symmetric tensors}

As the local representation of jets involves iterated partial differentiation, symmetric tensors are of major importance. In these notes we review the basic properties of symmetric tensors and the relevant notation as we use in the main text.

A.1. Multi-index notation. Multi-index notation is very effective when high-order tensors are involved, as is the situation here. A multi-index $I$ of length $k$ is a $k$-tuple of positive integers, e.g., $I=i_{1} \ldots i_{k}$. Multi-indices will be denoted by upper-case roman letters and the associated indices will be denoted by the corresponding lower case letters as in the example above. For example, we may write the components $T_{i j k}$ of a third order tensor $T$ as $T_{I}=T_{i_{1} i_{2} i_{3}}$. The length of a multi-index $I=i_{1} \ldots i_{k}$ is denoted as the absolute value of the multi-index, i.e., $|I|=k$. We will use the summation convention for multi-indices so the contraction of two tensors may be written as $T^{I} S_{I}$. When a multi-index appears more than twice in a term, or twice but not diagonally, it is implied that the summation convention for that multi-index is not in effect.

Multi-indices may be concatenated naturally so that for two multi-indices $I$ and $J$, the concatenated multi-index is $I J=i_{1} \ldots i_{|I|} j_{1} \ldots j_{|J|}$ whose length is $|I J|=|I|+|J|$. Thus, for two tensors $S_{I}$ and $T_{J}$, one may write $(S \otimes T)_{I J}=S_{I} T_{J}$.

For two multi-indices $I, J$, with $|I|=|J|=l$, one extends the definition of the Kronecker $\delta$ by

$$
\delta_{J}^{I}:=\delta_{j_{1}}^{i_{1}} \cdots \delta_{j_{l}}^{i_{l}}
$$

A.2. Symmetric tensors and permutations. Because of the commutativity of partial derivatives that we encounter frequently here, tensors that are completely symmetric are of particular interest. A tensor $T$ is completely symmetric if for any exchange of two indices $i_{r}$ and $i_{s}, T_{i_{1} \cdots i_{r} \cdots i_{s} \cdots i_{k}}=T_{i_{1} \cdots i_{s} \cdots i_{r} \cdots i_{k}}$. 
Symmetry can also be defined in terms of permutations. A permutation of the finite ordered set $(1, \ldots, l)$ is a bijection

$$
p:(1, \ldots, l) \rightarrow(1, \ldots, l) .
$$

The collection of all such permutations will be denoted by $\mathscr{P}_{l}$. From elementary combinatorics it follows that there are $l$ ! permutations in $\mathscr{P}_{l}$. For a multi-index $I$ and a permutation $p$, we set

$$
p(I):=I \circ p=i_{p(1)} \cdots i_{p(l)} .
$$

Note that $i_{p(r)}$ identifies the index that arrived under the permutation at the $r$-th position, while $i_{p^{-1}(s)}$ is the position of $i_{s}$ after the permutation $p$. Note also that we make some abuse of notation by using the same symbol for the permutation and its action on multi-indices. It immediately follows that for two permutations $p_{1}, p_{2} \in \mathscr{P}_{l}$,

$$
p_{2} \circ p_{1}(I)=I \circ p_{1} \circ p_{2} .
$$

Thus, using the language of permutations, a tensor is symmetric if for every permutation $p \in \mathscr{P}_{l}$,

$$
T_{p(I)}=T_{I} .
$$

Remark 1. We have defined symmetry above in terms of the components of the array representing a tensor. Viewed as a multilinear mapping, a (covariant) tensor $T$ is symmetric if

$$
T\left(v_{1}, \ldots, v_{l}\right)=T\left(v_{p(1)}, \ldots, v_{p(l)}\right)
$$

for any permutation $p$. In particular, for a symmetric tensor

$$
T_{i_{1} \cdots i_{1}}=T\left(\boldsymbol{e}_{i_{1}}, \ldots, \boldsymbol{e}_{i_{l}}\right)=T\left(\boldsymbol{e}_{p\left(i_{1}\right)}, \ldots, \boldsymbol{e}_{p\left(i_{l}\right)}\right)=T_{p\left(i_{1}\right) \cdots p\left(i_{l}\right)}
$$

(see also [Greub 1978]).

We will use the notation $\otimes^{l} \boldsymbol{V}$ for the space of contravariant $l$-tensors and $\bigodot^{l} \boldsymbol{V}$ for the subspace of symmetric tensors. We will also identify a tensor $T \in \bigotimes^{l} \boldsymbol{V}$ with the (possibly symmetric) multilinear mapping $\boldsymbol{V}^{*} \times \cdots \times \boldsymbol{V}^{*} \rightarrow \mathbb{R}$ in the space of (respectively, symmetric) multilinear mappings $L^{l}\left(\boldsymbol{V}^{*}, \mathbb{R}\right)$ (respectively, $\left.L_{S}^{l}\left(\boldsymbol{V}^{*}, \mathbb{R}\right)\right)$. Thus, we make the identifications

$$
\bigotimes^{l} \boldsymbol{V} \simeq L^{l}\left(\boldsymbol{V}^{*}, \mathbb{R}\right), \quad \bigodot^{l} \boldsymbol{V} \simeq L_{S}^{l}\left(\boldsymbol{V}^{*}, \mathbb{R}\right) .
$$

The inclusion of the symmetric tensors will be denoted as

$$
\iota_{S}: \bigodot^{l} \boldsymbol{V} \simeq L_{S}^{l}\left(\boldsymbol{V}^{*}, \mathbb{R}\right) \rightarrow \bigotimes^{l} \boldsymbol{V} \simeq L^{l}\left(\boldsymbol{V}^{*}, \mathbb{R}\right) .
$$

An analogous notation and terminology will be used for covariant tensors. 
Remark 2. The Levi-Civita symbol satisfies ${ }^{1}$

$$
\varepsilon_{J}^{I}=\varepsilon_{j_{1} \ldots j_{m}}^{i_{1} \ldots i_{l}}= \begin{cases}(-1)^{p} & \text { if there is a permutation } p \text { with } J=p(I), \\ 0 & \text { otherwise. }\end{cases}
$$

Thus, we set

$$
|\varepsilon|_{J}^{I}:=\left|\varepsilon_{j_{1} \ldots j_{m}}^{i_{1} \ldots i_{l}}\right|= \begin{cases}1 & \text { if there is a permutation } p \text { with } J=p(I), \\ 0 & \text { otherwise. }\end{cases}
$$

In particular,

$$
|\varepsilon|_{J}^{p(I)}=|\varepsilon|_{J}^{I}
$$

A.3. Cardinality sequence of a multi-index. A multi-index $I$ induces another sequence $\left(I_{1}, \ldots, I_{n}\right), n=\operatorname{dim} \boldsymbol{V}$, the cardinality sequence, in which $I_{r}$ indicates the number of times $r$ is included in the multi-index. Evidently, $|I|=\sum_{r=1}^{n} I_{r}$ and the sequence $\left(I_{1}, \ldots, I_{n}\right)$ is invariant under permutations of the multi-index.

A collection $\left(I_{1}, \ldots, I_{n}\right)$ induces a unique nondecreasing multi-index, i.e., the multi-index

$$
1 \cdots 12 \cdots 2 \cdots \cdots n \cdots n
$$

where the number $r$ appears $I_{r}$ times. Thus, if $\left(I_{1}, \ldots, I_{n}\right)$ is the cardinality sequence of $I$, we obtain a nondecreasing permutation of $I$.

For a multi-index $I$ it is useful to write

$$
I !=I_{1} ! \cdots I_{n} !
$$

It is observed that for a concatenated index $I J$, one has $(I J)_{r}=I_{r}+J_{r}, r=1, \ldots, n$. The index $i=1, \ldots, n$, is a simple multi-index $I=i$. Obviously $|I|=1$ and

$$
I_{r}= \begin{cases}0 & \text { for } r \neq i \\ 1 & \text { for } r=i\end{cases}
$$

Thus, for the concatenated multi-index $J i$, one has $(J i)_{r}=J_{r}+\delta_{r i}$, where $\delta$ is the Kronecker symbol.

For tensors that are symmetric with respect to a multi-index, a particular component is indicated uniquely by a sequence in the form $\left(I_{1}, \ldots, I_{n}\right)$ and by restricting the sequences $\left(i_{1}, \ldots, i_{|\boldsymbol{I}|}\right)$ to be nondecreasing. Consequently, we will use multiindices indicated by bold characters to be nondecreasing only and we will also write $\boldsymbol{I}=\left(I_{1}, \ldots, I_{n}\right)$. In addition, the fact that a multi-index is nondecreasing will be indicated by angle brackets, e.g., $T_{\langle J\rangle}$ or $T_{\langle\boldsymbol{I} \boldsymbol{J}\rangle}$, independently of the symmetry property of a tensor.

${ }^{1}$ This is a somewhat generalized presentation of the Levi-Civita symbol, for which see [LeviCivita 1927, p. 158]. 
A.4. Derivatives. Nondecreasing multi-indices are primarily used for notation involving partial derivatives. We will use the notation

$$
(\cdot)_{, \boldsymbol{I}}=\partial_{\boldsymbol{I}}(\cdot)=\frac{\partial^{|\boldsymbol{I}|}(\cdot)}{\partial x^{\boldsymbol{I}}}=\frac{\partial^{|\boldsymbol{I}|}(\cdot)}{\partial x^{i_{1}} \cdots \partial x^{i_{|I|}}}=\frac{\partial^{|\boldsymbol{I}|}(\cdot)}{\left(\partial x^{1}\right)^{I_{1}} \cdots\left(\partial x^{n}\right)^{I_{n}}} .
$$

Nondecreasing multi-indices may be added naturally by setting

$$
\boldsymbol{I}+\boldsymbol{J}=\left(I_{1}+J_{1}, \ldots, I_{n}+J_{n}\right),
$$

which determines a unique nondecreasing multi-index such that $|\boldsymbol{I}+\boldsymbol{J}|=|\boldsymbol{I}|+|\boldsymbol{J}|$. In particular,

$$
\left((\cdot)_{, \boldsymbol{I}}\right)_{, \boldsymbol{J}}=(\cdot)_{,\langle\boldsymbol{I} \boldsymbol{J}\rangle}=(\cdot),\langle\boldsymbol{I}+\boldsymbol{J}\rangle \cdot
$$

Nondecreasing multi-indices can also be partially ordered so that

$$
\boldsymbol{J} \leq \boldsymbol{I} \quad \Longleftrightarrow \quad J_{r} \leq I_{r} \text { for } r=1, \ldots, n .
$$

In the case $\boldsymbol{J} \leq \boldsymbol{I}$, one can use the subtraction $\boldsymbol{I}-\boldsymbol{J}$.

As hinted in the notation for partial derivatives, for $x \in \mathbb{R}^{n}$, one defines for a nondecreasing multi-index $\boldsymbol{I}$,

$$
x^{I}=\left(x^{1}\right)^{I_{1}} \cdots\left(x^{n}\right)^{I_{n}} .
$$

The summation convention will be applied for bold faced multi-indices, accordingly, only to the nondecreasing sequences. For example, a polynomial $\mathbb{R}^{n} \rightarrow \mathbb{R}$ of order $l$ may be written as

$$
u=a_{I} x^{I}, \quad 0 \leq|\boldsymbol{I}| \leq l .
$$

Suspending the summation convention, its derivatives are

$$
u, \boldsymbol{J}=\sum_{0 \leq|\boldsymbol{I}| \leq l} \frac{\boldsymbol{I} !}{(\boldsymbol{I}-\boldsymbol{J}) !} x^{\boldsymbol{I}-\boldsymbol{J}} .
$$

Although this relation is used mainly for the case where $J_{r} \leq I_{r}$, for all $r=1, \ldots, n$, it may be extended to all other cases by adopting the convention that

$$
\frac{1}{i !}=0 \quad \text { for } i<0 \text {. }
$$

The notation introduced above allows one to write the $l$-th order Taylor expansion of a function $f: \mathbb{R}^{n} \rightarrow \mathbb{R}$ in the form

$$
\sum_{0 \leq \boldsymbol{I} \leq l} \frac{1}{\boldsymbol{I} !} f_{, \boldsymbol{I}}(x) h^{\boldsymbol{I}} .
$$

To use the summation convention, one first sets $g_{\boldsymbol{I}}:=f_{, \boldsymbol{I}} / \boldsymbol{I}$ ! (no sum), and so the polynomial is written as

$$
g_{I} h^{I}, \quad 0 \leq|\boldsymbol{I}| \leq l .
$$


A.5. More on permutations. One observes that, for some given $I,|I|=l$, the sum

$$
\sum_{p \in \mathscr{P}_{l}} T_{p(I)}
$$

contains $l !=|I|$ ! terms, the number of all permutations. These include $I$ ! permutations (see below) that leave $I$ invariant. In the particular case where $T$ is symmetric,

$$
\sum_{p \in \mathscr{P}_{l}} T_{p(I)}=|I| ! T_{I}, \quad \text { no sum on } \boldsymbol{I} .
$$

On the other hand, in the expression

$$
|\varepsilon|_{I}^{J} T_{J}=\sum_{J, J=p(I)} T_{J}
$$

the sum applies only to possible values of the multi-index $J$, irrespective of the number of permutations of $I$ that give it. Assume that $J$ is a permutation of $I$ so that $|\varepsilon|_{I}^{J}=1$. As both $I$ and $J$ contain $I_{r}$ occurrences of the index $r$, permutations of which leave a multi-index invariant, there are $J !=J_{1} ! \cdots J_{l} !=I$ ! such permutations for each $J$. Since there are $I$ ! permutations that give any one particular multi-index $J$ if $|\varepsilon|_{I}^{J} \neq 0$, it follows that for any fixed $J$,

$$
\sum_{\substack{p(I)=J, p \in \mathscr{P}_{l}}} T_{p(I)}=I ! T_{J}=I !|\varepsilon|_{I}^{J} T_{J}, \quad \text { no sum on } I, J,
$$

and so

$$
\sum_{p \in \mathscr{P}_{l}} T_{p(I)}=\sum_{J}\left(\sum_{\substack{p(I)=J, p \in \mathscr{P}_{l}}} T_{p(I)}\right)=I !|\varepsilon|_{I}^{J} T_{J}, \quad \text { no sum on } I .
$$

We conclude that the number of nontrivial terms in the sum $|\varepsilon|_{I}^{J} T_{J}$ is

$$
\sum_{J}|\varepsilon|_{I}^{J}=\frac{|I| !}{I !}=\frac{l !}{I !} .
$$

In the particular case where $T$ is symmetric, $\sum_{p \in \mathscr{P}_{l}} T_{p(I)}=|I| ! T_{I}$, so that (A-30) implies immediately that

$$
\sum_{J, J=p(I)} T_{J}=|\varepsilon|_{I}^{J} T_{J}=\frac{|I| !}{I !} T_{I}
$$

For a given pair of multi-indices, $I, J$, and a variable permutation $p$,

$$
\delta_{p(J)}^{I}=|\varepsilon|_{J}^{I} .
$$


As a result

$$
\sum_{p \in \mathscr{P}_{l}} \delta_{p(J)}^{I}=I !|\varepsilon|_{J}^{I}, \quad \text { no sum on } I .
$$

Remark 3. For each nondecreasing multi-index $\boldsymbol{I},|\boldsymbol{I}|=l$, there are $|\boldsymbol{I}| ! / \boldsymbol{I}$ ! distinct indices $J$. Thus, the total number of distinct multi-indices is

$$
\sum_{I} \frac{\left(I_{1}+\cdots+I_{n}\right) !}{I_{1} ! \cdots I_{n} !}=n^{l},
$$

in accordance with the multinomial formula.

A.6. Symmetrization of arrays and tensors. Any $l$-tensor $T$, having the components $T_{I}$, induces a unique symmetric array, the components of which are denoted as $T_{(I)}$, by

$$
T_{(I)}=\sum_{p \in \mathscr{P}_{l}} \frac{1}{l !} T_{p(I)}=\frac{I !}{l !}|\varepsilon|_{I}^{J} T_{J}, \quad \text { no sum on } \boldsymbol{I} .
$$

We first show that $T_{(I)}$ is indeed symmetric. One has,

$$
T_{(q(I))}=\sum_{p \in \mathscr{P}_{l}} \frac{1}{l !} T_{p(q(I))}=\sum_{p \in \mathscr{P}_{l}} \frac{1}{l !} T_{p(I)}=T_{(I)},
$$

where in the second equality we used the fact that in the first equality we add up the terms over all permutations anyhow. One also observes that symmetrization is a projection in the sense that the symmetrization of a symmetric tensor yields the tensor itself. That is, if $T_{I}$ is symmetric,

$$
T_{(I)}=\sum_{p \in \mathscr{P}_{l}} \frac{1}{l !} T_{p(I)}=\sum_{p \in \mathscr{P}_{l}} \frac{1}{l !} T_{I}=T_{I} .
$$

The symmetrization of a multilinear mapping $T$ - a covariant tensor - is defined as the linear mapping

such that

$$
\mathscr{S}: \bigotimes^{l} \boldsymbol{V}^{*} \rightarrow \bigodot^{l} \boldsymbol{V}^{*}
$$

$$
(\mathscr{S}(T))\left(v_{1}, \ldots, v_{l}\right)=\frac{1}{l !} \sum_{p \in \mathscr{P}_{l}} T\left(v_{p(1)}, \ldots, v_{p(l)}\right) .
$$

In particular,

$$
\begin{aligned}
\mathscr{S}\left(\boldsymbol{e}^{I}\right)\left(v_{1}, \ldots, v_{l}\right) & :=\mathscr{Y}\left(\boldsymbol{e}^{i_{1}} \otimes \cdots \otimes \boldsymbol{e}^{i_{l}}\right)\left(v_{1}, \ldots, v_{l}\right) \\
& =\frac{1}{l !} \sum_{p \in \mathscr{P}_{l}}\left(\boldsymbol{e}^{i_{1}} \otimes \cdots \otimes \boldsymbol{e}^{i_{l}}\right)\left(v_{p(1)}, \ldots, v_{p(l)}\right) \\
& =\frac{1}{l !} \sum_{p \in \mathscr{P}_{l}}\left(\boldsymbol{e}^{p\left(i_{1}\right)} \otimes \cdots \otimes \boldsymbol{e}^{p\left(i_{l}\right)}\right)\left(v_{1}, \ldots, v_{l}\right),
\end{aligned}
$$


and it follows that (see [Greub 1978, p. 219])

$$
\mathscr{S}\left(\boldsymbol{e}^{I}\right)=\frac{1}{l !} \sum_{p \in \mathscr{P}_{l}}\left(\boldsymbol{e}^{p\left(i_{1}\right)} \otimes \cdots \otimes \boldsymbol{e}^{p\left(i_{l}\right)}\right)=: \boldsymbol{e}^{i_{1}} \odot \cdots \odot \boldsymbol{e}^{i_{l}}=: \bigodot^{I} \boldsymbol{e}^{I}=: \boldsymbol{e}^{(I)} .
$$

From this definition it follows immediately that

$$
\boldsymbol{e}_{(p(J))}=\boldsymbol{e}_{(J)} \text { for all } \quad p \in \mathscr{P} .
$$

Hence, $\boldsymbol{e}_{(J)}$ as well as all $\boldsymbol{e}_{(p(J))}$ are represented by the nondecreasing multi-index $\boldsymbol{J}=\langle\boldsymbol{J}\rangle=\left(J_{1}, \ldots, J_{n}\right)$.

Note that for a permutation $p \in \mathscr{P}_{l}$ and a multilinear mapping $T$, one may write $p T$ for the multilinear mapping defined by

$$
(p T)\left(v_{1}, \ldots, v_{l}\right):=T\left(v_{p(1)}, \ldots, v_{p(l)}\right) .
$$

Thus,

$$
\mathscr{S}(T)=\frac{1}{l !} \sum_{p \in \mathscr{P}_{l}} p T, \quad \boldsymbol{e}^{(I)}=\frac{1}{l !} \sum_{p \in \mathscr{P}_{l}} p \boldsymbol{e}^{I}=\frac{1}{l !} \sum_{p \in \mathscr{P}_{l}} \boldsymbol{e}^{p(I)} .
$$

The inclusion of the subspace of symmetric tensors will be denoted by

$$
\iota_{S}: \bigodot^{l} \boldsymbol{V}^{*} \rightarrow \bigotimes^{l} \boldsymbol{V}^{*} .
$$

Since the symmetrization of a symmetric tensor gives the original tensor, the symmetrization mapping $\mathscr{S}$ is a left inverse of the inclusion, i.e., $\mathscr{S} \circ \iota_{S}=\mathrm{Id}$.

It is readily verified that the array $\mathscr{S}(T)_{I}$ of a symmetrized multilinear mapping is the symmetrized array $T_{(I)}$.

A.7. Bases and dimension. We consider a vector space $\boldsymbol{V}$ with some basis $\left\{\boldsymbol{e}_{i}\right\}$, $i=1, \ldots, n$. Let $T$ be a (say contravariant) tensor $T$ of degree $l$ represented in the form

$$
T=T^{i_{1} \cdots i_{l}} \boldsymbol{e}_{i_{1}} \otimes \cdots \otimes \boldsymbol{e}_{i_{l}} .
$$

Using multi-index notation,

$$
T=T^{I} \boldsymbol{e}_{l}, \quad|I|=l,
$$

where

$$
\boldsymbol{e}_{I}:=\boldsymbol{e}_{i_{1}} \otimes \cdots \otimes \boldsymbol{e}_{i_{l}} .
$$

In particular, the dimension of the space is $n$.

The array of a symmetric tensor is uniquely determined by its components $T^{\boldsymbol{I}}$ for nondecreasing multi-indices only. Thus, the dimension of the space of symmetric $l$-tensors is obviously smaller. Since a nondecreasing $\boldsymbol{I}$ is uniquely determined by $I_{1}, \ldots, I_{n}$, the dimension may be determined accordingly. 
It is easy to realize that the number of independent component in a symmetric $l$-tensor is $C(n+l-1, l)=(n+l-1) ! /(n-1) ! l !$. One considers a string of $l$ nondecreasing indices, $I_{1}$ occurrences of $1, I_{2}$ occurrences of 2 , etc., where the end of each such group (except for the last one) is indicated by a divider. Thus, the number of distinct nondecreasing multi-indices is the number of different ways one can place the $n-1$ (identical) dividers in the string containing $l+n-1$ elements (both indices and dividers). It follows that the dimension of the space of symmetric $l$-tensors is $C(n+l-1, l)$.

Since a symmetric tensor is represented by a symmetric array,

$$
\begin{aligned}
T & =T^{I} \boldsymbol{e}_{I}=T^{(I)} \boldsymbol{e}_{I}=\frac{1}{l !} \sum_{p \in \mathscr{P}_{l}} T^{p(I)} \boldsymbol{e}_{I} \\
& =\frac{1}{l !} \sum_{p \in \mathscr{P}_{l}} T^{J} \boldsymbol{e}_{p^{-1}(J)}=\frac{1}{l !} T^{J} \sum_{q \in \mathscr{P}_{l}} \boldsymbol{e}_{q(J)}=T^{J} \boldsymbol{e}_{(J)},
\end{aligned}
$$

where in the second line we used the fact that the order of the sum of the multi-index and the sum over the group of permutations may be reversed. Here, in accordance with (A-42),

$$
\boldsymbol{e}_{(J)}=\bigodot^{J} \boldsymbol{e}_{J}:=\frac{1}{l !} \sum_{q \in \mathscr{P}_{l}} \boldsymbol{e}_{q(J)},
$$

or explicitly

$$
\boldsymbol{e}_{(J)}=\boldsymbol{e}_{j_{1}} \odot \cdots \odot \boldsymbol{e}_{j_{l}}:=\frac{1}{l !} \sum_{q \in \mathscr{P}_{l}} \boldsymbol{e}_{j_{q(1)}} \otimes \cdots \otimes \boldsymbol{e}_{j_{q(l)}},
$$

denotes the symmetric tensor product (see [Greub 1978, p. 219]).

Furthermore,

$$
\begin{aligned}
T & =T^{J} \boldsymbol{e}_{(J)} \\
& =\sum_{\boldsymbol{I}} \sum_{\substack{J=p(\boldsymbol{I}) \\
p \in \mathscr{P}_{l}}} T^{J} \boldsymbol{e}_{(J)} \quad(\text { no sum }) \\
& =\sum_{\boldsymbol{I}} \sum_{\substack{J=p(\boldsymbol{I}) \\
p \in \mathscr{P}_{l}}} T^{J} \boldsymbol{e}_{(\boldsymbol{I})} \quad\left(\text { as } \boldsymbol{e}_{(p(\boldsymbol{I}))}=\boldsymbol{e}_{(\boldsymbol{I})}\right) \\
& =\sum_{\boldsymbol{I}}\left(\sum_{\substack{J=p(\boldsymbol{I}) \\
p \in \mathscr{P}_{l}}} T^{J}\right) \boldsymbol{e}_{(\boldsymbol{I})} \\
& \left.=\sum_{\boldsymbol{I}} \frac{|\boldsymbol{I}| !}{\boldsymbol{I} !} T^{\boldsymbol{I} \boldsymbol{e}_{(\boldsymbol{I})}} \quad \text { (by }(\mathrm{A}-32)\right) .
\end{aligned}
$$


The last expression suggests that we make the definitions

$$
\begin{aligned}
\overleftarrow{\boldsymbol{e}}_{(\boldsymbol{I})}:=\frac{|\boldsymbol{I}| !}{\boldsymbol{I} !} \boldsymbol{e}_{(I)}, \quad \overleftarrow{T}^{\boldsymbol{I}}:=\frac{|\boldsymbol{I}| !}{\boldsymbol{I} !} T^{\boldsymbol{I}}, \\
\overrightarrow{\boldsymbol{e}}_{(J)}:=\frac{J !}{|J| !} \boldsymbol{e}_{(J)}, \quad \vec{T}^{J}:=\frac{J !}{|J| !} T^{J}
\end{aligned}
$$

and it is noted that the fractions $J ! /|J|$ ! are identical for all $J=p(\boldsymbol{I}), p \in \mathscr{P}$. Utilizing the summation convention again, we may write

$$
\begin{gathered}
T=T^{J} \boldsymbol{e}_{(J)}=T^{I} \overleftarrow{\boldsymbol{e}}_{(\boldsymbol{I})}=\overleftarrow{T}^{\boldsymbol{I}} \boldsymbol{e}_{(\boldsymbol{I})} \\
T^{J} \overrightarrow{\boldsymbol{e}}_{(J)}=\vec{T}^{J} \boldsymbol{e}_{(J)}=T^{\boldsymbol{I}} \boldsymbol{e}_{(\boldsymbol{I})}
\end{gathered}
$$

Evidently, both $\left\{\boldsymbol{e}_{(I)}\right\}$, and $\left\{\overleftarrow{\boldsymbol{e}}_{(\boldsymbol{I})}\right\}$ are collections of linearly independent tensors and may serve as bases for the space of symmetric tensors (see [Greub 1978; Comon et al. 2008, p. 219]). The components of the tensor relative to these bases change accordingly. The representation of a symmetric tensor in (A-47) is in terms of regular tensor products and is inadequate because these tensor products are not elements of the space of symmetric tensors, in general, and because it uses more elements than the dimension of the space. The appropriate representation of symmetric tensors in terms of base elements is given by (A-56).

Example 4. We consider now the inclusion

$$
\iota_{S}: \bigodot^{l} \boldsymbol{V} \rightarrow \bigotimes^{l} \boldsymbol{V}
$$

The matrix of the inclusion relative to the bases $\boldsymbol{e}_{J}$ in $\bigotimes^{l} \boldsymbol{V}$ and $\overleftarrow{\boldsymbol{e}}_{(\boldsymbol{I})}$ in $\bigodot^{l} \boldsymbol{V}$ satisfies

$$
\begin{array}{rlr}
\left(\iota_{S}\right)_{\boldsymbol{I}}^{J} \boldsymbol{e}_{J} & =\iota_{S}\left(\overleftarrow{\boldsymbol{e}}_{(\boldsymbol{I})}\right)=\frac{|\boldsymbol{I}| !}{\boldsymbol{I} !} \iota_{S}\left(\boldsymbol{e}_{(\boldsymbol{I})}\right)=\frac{|\boldsymbol{I}| !}{\boldsymbol{I} !} \boldsymbol{e}_{(\boldsymbol{I})} \\
& =\frac{1}{\boldsymbol{I} !} \sum_{p \in \mathscr{P}_{l}} \boldsymbol{e}_{p(\boldsymbol{I})} \quad(\text { no sum on } \boldsymbol{I}) \\
& =\frac{1}{\boldsymbol{I} !} \sum_{p \in \mathscr{P}_{l}} \delta_{p(\boldsymbol{I})}^{J} \boldsymbol{e}_{J} & \text { (no sum on } \boldsymbol{I}) \\
& =\frac{\boldsymbol{I} !}{\boldsymbol{I} !}|\varepsilon|_{\boldsymbol{I}}^{J} \boldsymbol{e}_{J} \quad \text { (using (A-34)) }
\end{array}
$$

It is concluded that

$$
\left(\iota_{S}\right)_{I}^{J}=|\varepsilon|_{I}^{J} .
$$

In addition, as the components of $T$ relative to the basis $\left\{\overleftarrow{\boldsymbol{e}}_{(I)}\right\}$ are $T^{\boldsymbol{I}}$

or

$$
\iota_{S}(T)=\left(\iota_{S}\right)_{\boldsymbol{I}}^{J} T^{\boldsymbol{I}} \boldsymbol{e}_{J}=|\varepsilon|_{I}^{J} T^{\boldsymbol{I}} \boldsymbol{e}_{J},
$$

$$
T^{J}=\left(\iota_{S}(T)\right)^{J}=|\varepsilon|_{I}^{J} T^{I},
$$


which could have been deduced otherwise.

Example 5. Consider the symmetrization mapping $\mathscr{Y}: \bigotimes^{l} \boldsymbol{V} \rightarrow \bigodot^{l} \boldsymbol{V}$. One has,

$$
\begin{aligned}
\mathscr{Y}\left(\boldsymbol{e}_{J}\right) & :=\boldsymbol{e}_{(J)} \\
& =|\varepsilon|_{J}^{\boldsymbol{I}} \boldsymbol{e}_{(\boldsymbol{I})} \quad(\text { only one } \boldsymbol{I}) \\
& =|\varepsilon|_{J}^{\boldsymbol{I}} \frac{\boldsymbol{I} !}{|\boldsymbol{I}| !} \overleftarrow{\boldsymbol{e}}_{(\boldsymbol{I})}
\end{aligned}
$$

and it follows from the definition of a matrix that

In addition,

$$
\mathscr{Y}_{J}^{\boldsymbol{I}}=\frac{\boldsymbol{I} !}{|\boldsymbol{I}| !}|\varepsilon|_{J}^{\boldsymbol{I}} .
$$

$$
\begin{aligned}
\mathscr{S}(T)^{\boldsymbol{I}}=\mathscr{Y}_{J}^{\boldsymbol{I}} T^{J} & =\frac{\boldsymbol{I} !}{|\boldsymbol{I}| !}|\varepsilon|_{J}^{\boldsymbol{I}} T^{J} \\
& =T^{(\boldsymbol{I})} \quad(\text { using (A-36)). }
\end{aligned}
$$

A.8. Duality. Consider the dual basis $\left\{\boldsymbol{e}^{i}\right\}$ of the dual vector space $\boldsymbol{V}^{*}$ so that $\boldsymbol{e}^{i}\left(\boldsymbol{e}_{j}\right)=\delta_{j}^{i}$. For any two multi-indices $I, J$, with $|I|=|J|=l$, we consider the action $\boldsymbol{e}^{(I)}\left(\boldsymbol{e}_{(J)}\right)$. We have

$$
\begin{aligned}
\boldsymbol{e}^{(I)}\left(\boldsymbol{e}_{(J)}\right) & =\left(\boldsymbol{e}^{i_{1}} \odot \cdots \odot \boldsymbol{e}^{i_{l}}\right)\left(\boldsymbol{e}_{j_{1}} \odot \cdots \odot \boldsymbol{e}_{j_{l}}\right) \\
& =\frac{1}{(l !)^{2}}\left(\sum_{p \in \mathscr{P}_{l}} \boldsymbol{e}^{i_{p(1)}} \otimes \cdots \otimes \boldsymbol{e}^{i_{p(l)}}\right)\left(\sum_{q \in \mathscr{P}_{l}} \boldsymbol{e}_{j_{q(1)}} \otimes \cdots \otimes \boldsymbol{e}_{j_{q(l)}}\right) \\
& =\frac{1}{(l !)^{2}} \sum_{p \in \mathscr{P}_{l}}\left(\sum_{q \in \mathscr{P}_{l}} \delta_{q(J)}^{p(I)}\right) \\
& =\frac{1}{(l !)^{2}} \sum_{p \in \mathscr{P}_{l}} I !|\varepsilon|_{J}^{p(I)} \quad \text { (using (A-34)) } \\
& =\frac{I !}{(l !)^{2}} \sum_{p \in \mathscr{P}_{l}}|\varepsilon|_{J}^{I} \quad \text { (thing (A-12)) } \\
& =\frac{I ! l !}{(l !)^{2}}|\varepsilon|_{J}^{I} \\
& =\frac{I !}{|I| !}|\varepsilon|_{J}^{I} .
\end{aligned}
$$

It follows from the identity above that for nondecreasing multi-indices $I, J$,

$$
\boldsymbol{e}^{(\boldsymbol{I})}\left(\overleftarrow{\boldsymbol{e}}_{(\boldsymbol{J})}\right)=\boldsymbol{e}^{(\boldsymbol{I})}\left(\frac{|\boldsymbol{J}| !}{\boldsymbol{J} !} \boldsymbol{e}_{(\boldsymbol{J})}\right)=|\varepsilon|_{J}^{\boldsymbol{I}}=\delta_{\boldsymbol{J}}^{\boldsymbol{I}}
$$

where one realizes that if the two multi-indices are nondecreasing, one can be a permutation of the other only when they are equal. 
The last identity implies that the basis $\left\{\boldsymbol{e}^{(\boldsymbol{I})}\right\}$ is the dual basis of $\left\{\overleftarrow{\boldsymbol{e}}_{(\boldsymbol{J})}\right\}$, and in particular,

$$
\bigodot^{l} \boldsymbol{V}^{*} \simeq\left(\bigodot^{l} \boldsymbol{V}\right)^{*}
$$

Finally, for $T=T^{\boldsymbol{I}} \overleftarrow{\boldsymbol{e}}_{(\boldsymbol{I})} \in \bigodot^{l} \boldsymbol{V}$ and $\psi=\psi_{\boldsymbol{I}} \boldsymbol{e}^{(\boldsymbol{I})} \in \bigodot^{l} \boldsymbol{V}^{*}$

$$
\psi(T)=\psi_{\boldsymbol{I}} T^{\boldsymbol{I}} .
$$

A.9. Symmetrization of cotensors and cosymmetrization. The inclusion of symmetric tensors in the collection of all tensors induces by duality a projection

$$
\iota_{S}^{*}:\left(\bigotimes^{l} \boldsymbol{V}\right)^{*} \simeq \bigotimes^{l} \boldsymbol{V}^{*} \rightarrow\left(\bigodot^{l} \boldsymbol{V}\right)^{*} \simeq \bigodot^{l} \boldsymbol{V}^{*},
$$

such that

$$
\iota_{S}^{*}(\varphi)(T)=\varphi(\iota(T)),
$$

for every symmetric tensor $T$. Thus, referring to elements of $\left(\otimes^{l} \boldsymbol{V}\right)^{*}$ as cotensors, $\iota_{S}^{*}$ is a symmetrization operator for cotensors.

One obtains

$$
\left(\iota_{S}^{*}(\varphi)\right)_{I}=\left(\iota_{S}^{*}\right)_{I}^{J} \varphi_{J}=|\varepsilon|_{I}^{J} \varphi_{J},
$$

where we observe that in the last expression one adds up the components of $\varphi$ corresponding to all permutations of $\boldsymbol{I}$, similarly to the symmetrization operation (but without taking the average).

In addition,

$$
\iota_{S}^{*}(\varphi)(T)=\left(\iota_{S}^{*}(\varphi)\right)_{I} T^{I}=|\varepsilon|_{I}^{J} \varphi_{J} T^{I}=\varphi_{J} T^{J},
$$

as expected. In the particular case where $\varphi$ is symmetric, using (A-32) and (A-72) gives

$$
\left(\iota_{S}^{*}(\varphi)\right)_{\boldsymbol{I}}=|\varepsilon|_{\boldsymbol{I}}^{J} \varphi_{J}=\frac{|\boldsymbol{I}| !}{\boldsymbol{I} !} \varphi_{\boldsymbol{I}},
$$

and

$$
\iota_{S}^{*}(\varphi)(T)=\varphi_{J} T^{J}=\sum_{\boldsymbol{I}} \frac{|\boldsymbol{I}| !}{\boldsymbol{I} !} \varphi_{\boldsymbol{I}} T^{\boldsymbol{I}} .
$$

The dual of the symmetrization mapping is (the cosymmetrization)

$$
\mathscr{\varphi}^{*}: \bigodot^{l} \boldsymbol{V}^{*} \rightarrow \bigotimes^{l} \boldsymbol{V}^{*}
$$

given by

$$
\mathscr{\varphi}^{*}(\psi)(T)=\psi(\mathscr{S}(T)) .
$$

Using the matrix obtained in Example 5, we have

$$
\mathscr{S}^{*}(\psi)(T)=\left(\mathscr{S}^{*}\right)_{J}^{\boldsymbol{I}} \psi_{\boldsymbol{I}} T^{J}=\sum_{\boldsymbol{I}, J} \frac{\boldsymbol{I} !}{|\boldsymbol{I}| !}|\varepsilon|_{J}^{\boldsymbol{I}} \psi_{\boldsymbol{I}} T^{J},
$$


and it follows that

$$
\mathscr{S}^{*}(\psi)_{J}=\sum_{\boldsymbol{I}} \frac{\boldsymbol{I} !}{|\boldsymbol{I}| !}|\varepsilon|_{J}^{\boldsymbol{I}} \psi_{\boldsymbol{I}}
$$

(It is observed that the sum over $\boldsymbol{I}$ contains only one nontrivial term.) In other words, if $J$ is a permutation of $\boldsymbol{I}$, then $\boldsymbol{I}=\langle J\rangle(\boldsymbol{I}$ is obtained by ordering $J)$, and

$$
\mathscr{S}^{*}(\psi)_{J}=\frac{\boldsymbol{I} !}{|\boldsymbol{I}| !} \psi_{\boldsymbol{I}}=\frac{J !}{|J| !} \psi_{\langle J\rangle} .
$$

In particular, if $T$ is symmetric, $\mathscr{S}^{*}(\psi)(T)=\psi(\mathscr{S}(T))=\psi(T)$, and so

$$
\sum_{J} \frac{J !}{|J| !} \psi_{\langle J\rangle} T^{J}=\sum_{I} \psi_{\boldsymbol{I}} T^{I}
$$

The last equation simply implies that for each nondecreasing $\boldsymbol{I}$ there are $|\boldsymbol{I}| ! / \boldsymbol{I} !=$ $|J| ! / J$ ! distinct indices $J$ obtained by permutations.

Setting

$$
\overleftarrow{T}^{I}:=\frac{|\boldsymbol{I}| !}{\boldsymbol{I} !} T^{\boldsymbol{I}}, \quad \stackrel{\rightarrow}{\rightarrow}^{K}:=\frac{K !}{|K| !} T^{K}
$$

one can write

$$
\psi_{\langle J\rangle} \stackrel{\vec{T}}{\rightarrow}^{J}=\sum_{I} \psi_{\boldsymbol{I}} T^{\boldsymbol{I}}, \quad \psi_{\langle J\rangle} T^{J}=\psi_{\boldsymbol{I}} \overleftarrow{\leftarrow}_{\leftarrow}^{\underline{I}}
$$

\section{References}

[Abraham et al. 1988] R. Abraham, J. E. Marsden, and T. Ratiu, Manifolds, tensor analysis, and applications, 2nd ed., Applied Mathematical Sciences 75, Springer, 1988.

[Comon et al. 2008] P. Comon, G. Golub, L.-H. Lim, and B. Mourrain, "Symmetric tensors and symmetric tensor rank", SIAM J. Matrix Anal. Appl. 30:3 (2008), 1254-1279.

[Greub 1978] W. Greub, Multilinear algebra, 2nd ed., Springer, 1978.

[Levi-Civita 1927] T. Levi-Civita, The absolute differential calculus (calculus of tensors), Blackie, London, 1927. Reprinted Dover, New York, 1977.

[Noll 1959] W. Noll, "The foundations of classical mechanics in the light of recent advances in continuum mechanics", pp. 266-281 in The axiomatic method, with special reference to geometry and physics (Berkeley, CA, 1957-1958), edited by P. S. L. Henkin and A. Tarski, North-Holland, Amsterdam, 1959.

[Palais 1968] R. S. Palais, Foundations of global non-linear analysis, W. A. Benjamin, New York, 1968.

[Saunders 1989] D. J. Saunders, The geometry of jet bundles, London Mathematical Society Lecture Note Series 142, Cambridge University Press, 1989.

[Segev 1986] R. Segev, "Forces and the existence of stresses in invariant continuum mechanics", $J$. Math. Phys. 27:1 (1986), 163-170.

[Segev 1994] R. Segev, "A geometrical framework for the statics of materials with microstructure", Math. Models Methods Appl. Sci. 4:6 (1994), 871-897.

[Segev 2000] R. Segev, "The geometry of Cauchy's fluxes", Arch. Ration. Mech. Anal. 154:3 (2000), 183-198. 
[Segev 2002] R. Segev, "Metric-independent analysis of the stress-energy tensor", J. Math. Phys. 43:6 (2002), 3220-3231.

[Segev 2013] R. Segev, "Notes on metric independent analysis of classical fields", Math. Methods Appl. Sci. 36:5 (2013), 497-566.

[Segev 2017] R. Segev, "Geometric analysis of hyper-stresses", Internat. J. Engrg. Sci. 120 (2017), $100-118$.

[Segev and Rodnay 1999] R. Segev and G. Rodnay, "Cauchy's theorem on manifolds", J. Elasticity 56:2 (1999), 129-144.

[Truesdell and Toupin 1960] C. Truesdell and R. Toupin, "The classical field theories", pp. 226-793; appendix, pp. 794-858 in Handbuch der Physik, vol. III/1, edited by S. Flügge, Springer, 1960.

Received 28 Oct 2017. Revised 8 Feb 2018. Accepted 21 Mar 2018.

REUVEN SEgEV: rsegev@bgu.ac.il

Department of Mechanical Engineering, Ben-Gurion University of the Negev, Beer-Sheva, Israel

JĘDRZEJ ŚNIATYCKI: sniatycki@gmail.com

Department of Mathematics and Statistics, University of Victoria, Victoria, BC, Canada and

Department of Mathematics and Statistics, University of Calgary, Calgary, AL, Canada 


\title{
AN ARBITRARILY SHAPED ESHELBY INCLUSION INTERACTING WITH A CIRCULAR PIEZOELECTRIC INHOMOGENEITY PENETRATED BY A SEMI-INFINITE CRACK
}

\author{
Xu Wang And Peter Schiavone
}

\begin{abstract}
We study the interaction between an Eshelby inclusion of arbitrary shape and a circular piezoelectric inhomogeneity penetrated by a semi-infinite crack under antiplane mechanical and in-plane electrical loading in a linear piezoelectric solid. The Eshelby inclusion undergoes uniform antiplane eigenstrains and inplane eigenelectric fields. Through the use of a conformal mapping, the cracked piezoelectric plane is first mapped onto the lower half of the image plane. The corresponding boundary value problem is then studied in this image plane. The interaction problem is solved through the construction of an auxiliary function and the application of analytic continuation across straight and circular boundaries. We obtain concise expressions for the resultant stress and electric displacement intensity factors at the crack tip.
\end{abstract}

\section{Introduction}

It is well-known that various kinds of defects, such as dislocations, cracks, Eshelby inclusions, and inhomogeneities can significantly affect the performance and integrity of piezoelectric devices. Theoretical analysis of these defects has continued to attract the attention of several researchers in the literature; see, for example, [Deeg 1980; Pak 1990; Kuo and Barnett 1991; Suo et al. 1992; Chung and Ting 1996; Lee et al. 2000; Ru 2000; 2001; Wang and Fan 2015; Wang and Schiavone 2017]. $\mathrm{Ru}[2000 ; 2001]$ used the technique of analytic continuation together with carefully constructed auxiliary functions in the physical plane to derive analytic solutions for Eshelby's problem of a two-dimensional Eshelby inclusion of arbitrary shape in a piezoelectric plane or half-plane or in one of two perfectly bonded piezoelectric half-planes. One example of the practical importance of Eshelby's problem lies in the study of residual stresses induced by lattice mismatch between buried active components and surrounding materials in strained semiconductor devices. It is

\section{Communicated by David J. Steigmann.}

MSC2010: 30E25, 74B05, 74G70.

Keywords: crack, Eshelby inclusion, inhomogeneity, piezoelectric material, analytic continuation, conformal mapping, field intensity factors. 
well-known that these residual stresses crucially affect the electronic performance of these devices and may lead to failure and degradation [Ru 2000; 2001].

This work investigates the Eshelby inclusion problem in fibrous piezoelectric composites containing cracks. In this paper, we endeavor to consider, simultaneously, within one single framework, the effects of a crack, an inhomogeneity, and an Eshelby inclusion in piezoelectric materials. More specifically, we study the antiplane shear deformations of an infinite hexagonal piezoelectric matrix containing

(i) a circular hexagonal piezoelectric inhomogeneity partially penetrated by a semi-infinite crack, and

(ii) an Eshelby inclusion of arbitrary shape undergoing uniform antiplane eigenstrains and in-plane eigenelectric fields; when the matrix is subjected to remote antiplane mechanical and in-plane electrical loading.

The piezoelectric plane weakened by the semi-infinite crack is first mapped onto the lower half of an image plane constructed via the use of a conformal mapping. The corresponding problem is then studied in this image plane. The construction of a specific auxiliary function and the application of analytic continuation across straight and circular boundaries lead to the derivation of analytic vector functions in each of the three phases of the fibrous piezoelectric composite. The resultant field intensity factors at the crack tip are also obtained. Our analysis indicates that when a condition on eigenstrains and eigenelectric fields is met, the Eshelby inclusion will exert a neutral effect (neither shielding nor antishielding) on the electroelastic field at the crack tip.

\section{Basic formulation}

In the case of antiplane shear deformations of a hexagonal piezoelectric material with poling direction along the $x_{3}$-axis, the general solution can be expressed in terms of a two-dimensional analytic vector function $\boldsymbol{f}(z)$ of the complex variable $z=x_{1}+i x_{2}$ as

$$
\begin{gathered}
{\left[\begin{array}{l}
\varphi_{1} \\
\varphi_{2}
\end{array}\right]+\mathrm{i} \boldsymbol{C}\left[\begin{array}{c}
u_{3} \\
\phi
\end{array}\right]=\boldsymbol{C} \boldsymbol{f}(z),} \\
{\left[\begin{array}{c}
2 \varepsilon_{32}+2 \mathrm{i} \varepsilon_{31} \\
-E_{2}-\mathrm{i} E_{1}
\end{array}\right]=\boldsymbol{f}^{\prime}(z), \quad\left[\begin{array}{c}
\sigma_{32}+\mathrm{i} \sigma_{31} \\
D_{2}+\mathrm{i} D_{1}
\end{array}\right]=\boldsymbol{C} \boldsymbol{f}^{\prime}(z),} \\
\boldsymbol{C}=\boldsymbol{C}^{T}=\left[\begin{array}{rr}
C_{44} & e_{15} \\
e_{15} & -\epsilon_{11}
\end{array}\right],
\end{gathered}
$$

where $\varphi_{1}$ and $\varphi_{2}$ are the stress function and charge potential, respectively; $u_{3}$ and $\phi$ are the antiplane displacement and electric potential, respectively; $\sigma_{31}$ and $\sigma_{32}$ are the antiplane shear stresses; $D_{1}$ and $D_{2}$ are electric displacements; $E_{1}$ and $E_{2}$ 
are in-plane electric fields; $\varepsilon_{31}$ and $\varepsilon_{32}$ are mechanical strains; $C_{44}, e_{15}$, and $\epsilon_{11}$ are the elastic, piezoelectric, and dielectric constants, respectively.

In addition, the stress function and the charge potential are defined in terms of the stresses and the electric displacements, respectively, by

$$
\sigma_{31}=-\varphi_{1,2}, \quad \sigma_{32}=\varphi_{1,1}, \quad D_{1}=-\varphi_{2,2}, \quad D_{2}=\varphi_{2,1} .
$$

\section{An Eshelby inclusion near a cracked circular piezoelectric inhomogeneity}

As shown in Figure 1, we consider an infinite hexagonal piezoelectric matrix containing an Eshelby inclusion of arbitrary shape undergoing uniform antiplane eigenstrains $\left(\varepsilon_{31}^{*}, \varepsilon_{32}^{*}\right)$ and in-plane eigenelectric fields $\left(E_{1}^{*}, E_{2}^{*}\right)$ as well as a circular hexagonal piezoelectric inhomogeneity. The poling directions of all three phases lie along the $x_{3}$-axis. A semi-infinite traction-free and charge-free crack partially penetrating the inhomogeneity lies on the negative real axis. The electroelastic constants of the matrix are identical to those of the inclusion but are different from those of the inhomogeneity. We represent the matrix by the domain $S_{2}$ and assume that the inhomogeneity occupies a circular region $S_{1}$ of radius $R$ with its center at the origin. The inclusion is assumed to occupy the region denoted by $S_{3}$. Both the inhomogeneity-matrix interface $|z|=R$ and the inclusion-matrix interface denoted here by $\Gamma$ are assumed to be perfectly bonded. Throughout the paper, the quantities in $S_{1}, S_{2}$, and $S_{3}$ will be identified by the subscripts 1,2 , and 3, respectively.

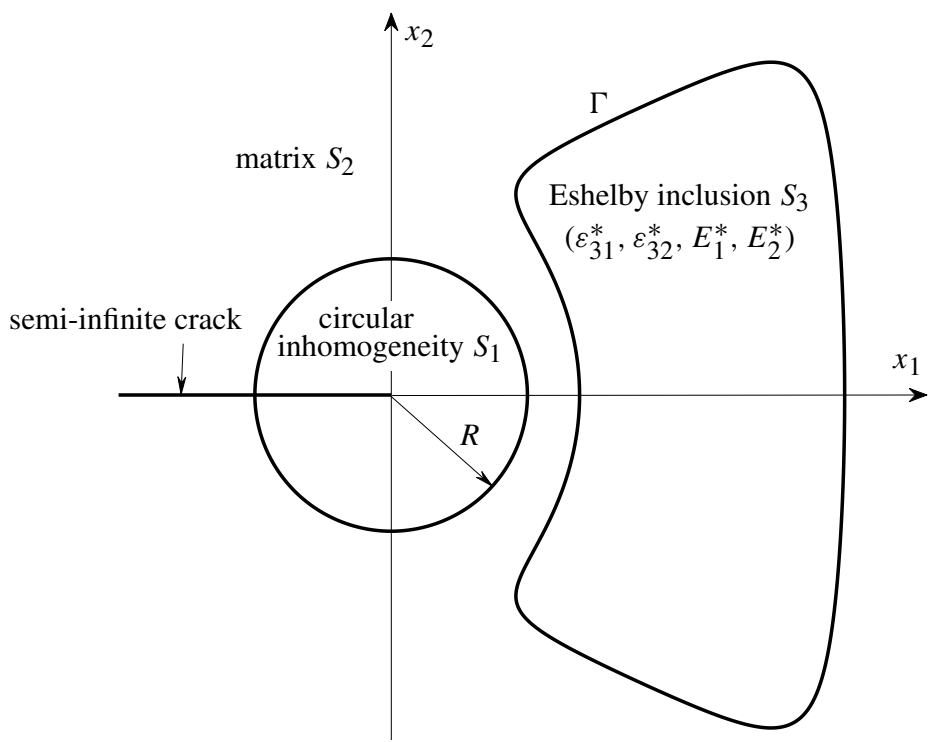

Figure 1. Interaction of an Eshelby inclusion of arbitrary shape with a circular piezoelectric inhomogeneity partially penetrated by a semi-infinite crack. 
In the physical $z$-plane, the boundary value problem has the form

$$
\begin{aligned}
& \boldsymbol{f}_{1}(z)+\overline{\boldsymbol{f}_{1}(z)}=\mathbf{0}, \quad-R<x_{1}<0, x_{2}=0^{ \pm} ; \\
& \boldsymbol{f}_{2}(z)+\overline{\boldsymbol{f}_{2}(z)}=\mathbf{0}, \quad-\infty<x_{1}<-R, \quad x_{2}=0^{ \pm} ; \\
& \boldsymbol{C}_{1} \boldsymbol{f}_{1}(z)+\boldsymbol{C}_{1} \overline{\boldsymbol{f}_{1}(z)}=\boldsymbol{C}_{2} \boldsymbol{f}_{2}(z)+\boldsymbol{C}_{2} \overline{\boldsymbol{f}_{2}(z)}, \\
& \boldsymbol{f}_{1}(z)-\overline{\boldsymbol{f}_{1}(z)}=\boldsymbol{f}_{2}(z)-\overline{\boldsymbol{f}_{2}(z)}, \quad|z|=R ;
\end{aligned}
$$

$$
\begin{aligned}
& \boldsymbol{f}_{2}(z)+\overline{\boldsymbol{f}_{2}(z)}= \boldsymbol{f}_{3}(z)+\overline{\boldsymbol{f}_{3}(z)}, \\
& \boldsymbol{f}_{2}(z)-\overline{\boldsymbol{f}_{2}(z)}= \boldsymbol{f}_{3}(z)-\overline{\boldsymbol{f}_{3}(z)}+\left[\begin{array}{c}
2\left(\varepsilon_{32}^{*}+\mathrm{i} \varepsilon_{31}^{*}\right) z-2\left(\varepsilon_{32}^{*}-\mathrm{i} \varepsilon_{31}^{*}\right) \bar{z} \\
-\left(E_{2}^{*}+\mathrm{i} E_{1}^{*}\right) z+\left(E_{2}^{*}-\mathrm{i} E_{1}^{*}\right) \bar{z}
\end{array}\right], \quad z \in \Gamma ; \\
& \boldsymbol{f}_{2}(z) \cong \sqrt{2 / \pi} \boldsymbol{C}_{2}^{-1} \boldsymbol{K} \sqrt{z}+O(1), \quad|z| \rightarrow \infty,
\end{aligned}
$$

where

$$
\boldsymbol{K}=\left[K^{\sigma} K^{D}\right]^{T},
$$

in which $K^{\sigma}$ and $K^{D}$ denote the stress and electric displacement intensity factors, respectively. These intensity factors represent the far-field electromechanical loads.

Consider the following conformal mapping function:

$$
z=\omega(\xi)=-\xi^{2}, \quad \xi=\omega^{-1}(z)=-\mathrm{i} \sqrt{z}, \quad \operatorname{Im}\{\xi\} \leq 0 .
$$

The physical $z$-plane with the semi-infinite crack is mapped onto the lower half$\xi$-plane and the crack faces are mapped onto the real axis in the $\xi$-plane. Moreover, the inhomogeneity $z \in S_{1}$ is mapped onto $\xi \in \Omega_{1}$; the matrix $z \in S_{2}$ is mapped onto $\xi \in \Omega_{2}$; the Eshelby inclusion $z \in S_{3}$ is mapped onto $\xi \in \Omega_{3}$; the inhomogeneity-matrix interface $|z|=R$ is mapped onto the semicircle $|\xi|=R^{1 / 2}$, $-\pi \leq \arg \{\xi\} \leq 0$; and, finally, the inclusion-matrix interface $z \in \Gamma$ is mapped onto $\xi \in L$ (see Figure 2).

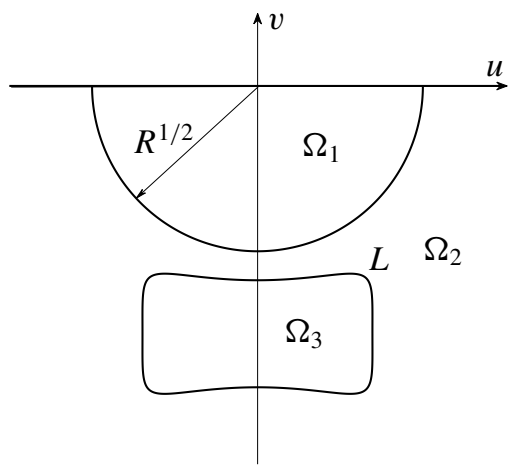

Figure 2. The problem in the $\xi$-plane. 
Without loss of generality, we write $f_{i}(\xi)=f_{i}(\omega(\xi)), i=1,2,3$ and denote by $u$ and $v$ the real and imaginary parts of $\xi$ (i.e., $\xi=u+\mathrm{i} v$ ). In the image $\xi$-plane, the boundary value problem takes the form

$$
\begin{gathered}
\boldsymbol{f}_{1}(\xi)+\overline{\boldsymbol{f}_{1}(\xi)}=\mathbf{0}, \quad|u|<R^{1 / 2}, \quad v=0^{-} ; \\
\boldsymbol{f}_{2}(\xi)+\overline{\boldsymbol{f}_{2}(\xi)}=\mathbf{0}, \quad R^{1 / 2}<|u|<\infty, \quad v=0^{-} ; \\
\boldsymbol{C}_{1} \boldsymbol{f}_{1}(\xi)+\boldsymbol{C}_{1} \overline{\boldsymbol{f}_{1}(\xi)}=\boldsymbol{C}_{2} \boldsymbol{f}_{2}(\xi)+\boldsymbol{C}_{2} \overline{\boldsymbol{f}_{2}(\xi)}, \\
\boldsymbol{f}_{1}(\xi)-\overline{\boldsymbol{f}_{1}(\xi)}=\boldsymbol{f}_{2}(\xi)-\overline{\boldsymbol{f}_{2}(\xi)}, \quad|\xi|=R^{1 / 2}, \quad-\pi \leq \arg \{\xi\} \leq 0 ; \\
\boldsymbol{f}_{2}(\xi)+\overline{\boldsymbol{f}_{2}(\xi)}=\boldsymbol{f}_{3}(\xi)+\overline{\boldsymbol{f}_{3}(\xi)}, \\
\boldsymbol{f}_{2}(\xi)-\overline{\boldsymbol{f}_{2}(\xi)}=\boldsymbol{f}_{3}(\xi)-\overline{\boldsymbol{f}_{3}(\xi)}+\left[\begin{array}{c}
-2\left(\varepsilon_{32}^{*}+\mathrm{i} \varepsilon_{31}^{*}\right) \xi^{2}+2\left(\varepsilon_{32}^{*}-\mathrm{i} \varepsilon_{31}^{*}\right) \bar{\xi}^{2} \\
\left(E_{2}^{*}+\mathrm{i} E_{1}^{*}\right) \xi^{2}-\left(E_{2}^{*}-\mathrm{i} E_{1}^{*}\right) \bar{\xi}^{2}
\end{array}\right], \quad \xi \in L ; \\
\boldsymbol{f}_{2}(\xi) \cong \mathrm{i} \sqrt{2 / \pi} \boldsymbol{C}_{2}^{-1} \boldsymbol{K} \xi+O(1), \quad|\xi| \rightarrow \infty .
\end{gathered}
$$

An analytic solution to the above boundary value problem appears to be extremely difficult to obtain since we have to handle the boundary conditions on $\operatorname{Im}\{\xi\}=0$, the interface conditions on $|\xi|=R^{1 / 2},-\pi \leq \arg \{\xi\} \leq 0$, and those on $\xi \in L$.

Adding the two interface conditions in $(8 \mathrm{c})$, we arrive at

$$
f_{2}(\xi)=f_{3}(\xi)-\xi^{2}\left[\begin{array}{c}
\varepsilon_{32}^{*}+\mathrm{i} \varepsilon_{31}^{*} \\
-\frac{1}{2}\left(E_{2}^{*}+\mathrm{i} E_{1}^{*}\right)
\end{array}\right]+\bar{\xi}^{2}\left[\begin{array}{c}
\varepsilon_{32}^{*}-\mathrm{i} \varepsilon_{31}^{*} \\
-\frac{1}{2}\left(E_{2}^{*}-\mathrm{i} E_{1}^{*}\right)
\end{array}\right], \quad \xi \in L
$$

The region $\xi \in \Omega_{3}$ is simply connected if $z \in S_{3}$ is simply connected. Consequently, there exists a conformal mapping $\xi=w(\eta)$ that maps the exterior of the simply connected region $\Omega_{3}$ in the $\xi$-plane onto the exterior of the unit circle $|\eta| \geq 1$ in the $\eta$-plane [Kantorovich and Krylov 1958; Savin 1961; England 1971]. As a result, an auxiliary function $D(\xi)$ can be constructed as follows:

$$
\bar{\xi}^{2}=\left[\bar{w}\left(\frac{1}{w^{-1}(\xi)}\right)\right]^{2}=D(\xi), \quad \xi \in L,
$$

where $w^{-1}(\xi)$ is the inverse mapping of $\xi=w(\eta)$.

Moreover, $D(\xi)$ is analytic in the exterior of $\Omega_{3}$ except at the point at infinity, where it has a pole of finite degree, namely

$$
D(\xi) \cong P(\xi)+O\left(\xi^{-1}\right), \quad|\xi| \rightarrow \infty
$$

where $P(\xi)$ is a polynomial of order $2 N$ in $\xi$ if $\xi=w(\eta)$ is a polynomial of order $N$ in $1 / \eta$. 
Using (10) and (11), Equation (9) can be written as

$$
\begin{aligned}
\boldsymbol{f}_{2}(\xi)- & {[D(\xi)-P(\xi)]\left[\begin{array}{c}
\varepsilon_{32}^{*}-\mathrm{i} \varepsilon_{31}^{*} \\
-\frac{1}{2}\left(E_{2}^{*}-\mathrm{i} E_{1}^{*}\right)
\end{array}\right] } \\
= & \boldsymbol{f}_{3}(\xi)-\xi^{2}\left[\begin{array}{c}
\varepsilon_{32}^{*}+\mathrm{i} \varepsilon_{31}^{*} \\
-\frac{1}{2}\left(E_{2}^{*}+\mathrm{i} E_{1}^{*}\right)
\end{array}\right]+P(\xi)\left[\begin{array}{c}
\varepsilon_{32}^{*}-\mathrm{i} \varepsilon_{31}^{*} \\
-\frac{1}{2}\left(E_{2}^{*}-\mathrm{i} E_{1}^{*}\right)
\end{array}\right], \quad \xi \in L .
\end{aligned}
$$

In view of (12), we introduce a new analytic vector function $\boldsymbol{h}(\xi)$ defined by

$$
\boldsymbol{h}(\xi)= \begin{cases}f_{2}(\xi)-[D(\xi)-P(\xi)]\left[\begin{array}{c}
\varepsilon_{32}^{*}-\mathrm{i} \varepsilon_{31}^{*} \\
-\frac{1}{2}\left(E_{2}^{*}-\mathrm{i} E_{1}^{*}\right)
\end{array}\right], & \xi \in \Omega_{2} ; \\
\boldsymbol{f}_{3}(\xi)-\xi^{2}\left[\begin{array}{c}
\varepsilon_{32}^{*}+\mathrm{i} \varepsilon_{31}^{*} \\
-\frac{1}{2}\left(E_{2}^{*}+\mathrm{i} E_{1}^{*}\right)
\end{array}\right]+P(\xi)\left[\begin{array}{c}
\varepsilon_{32}^{*}-\mathrm{i} \varepsilon_{31}^{*} \\
-\frac{1}{2}\left(E_{2}^{*}-\mathrm{i} E_{1}^{*}\right)
\end{array}\right], & \xi \in \Omega_{3} .\end{cases}
$$

We can see from the above definition and (12) that $\boldsymbol{h}(\xi)$ is continuous across $\xi \in L$ and is then analytic in $\xi \in \Omega_{2} \cup \Omega_{3}$ except at the point at infinity, where its asymptotic behavior is the same as that of $\boldsymbol{f}_{2}(\xi)$ given by (8d).

By satisfying the boundary conditions on $\operatorname{Im}\{\xi\}=0$ and the interface conditions on $|\xi|=R^{1 / 2},-\pi \leq \arg \{\xi\} \leq 0$, using analytic continuation across straight and circular boundaries, we finally arrive at the following expressions for $\boldsymbol{f}_{1}(\xi), \boldsymbol{f}_{2}(\xi)$, and $\boldsymbol{f}_{3}(\xi)$ :

$$
\begin{aligned}
\boldsymbol{f}_{1}(\xi)= & 2\left(\boldsymbol{C}_{1}+\boldsymbol{C}_{2}\right)^{-1} \boldsymbol{C}_{2} \\
\times & \left\{[D(\xi)-P(\xi)]\left[\begin{array}{c}
\varepsilon_{32}^{*}-\mathrm{i} \varepsilon_{31}^{*} \\
-\frac{1}{2}\left(E_{2}^{*}-\mathrm{i} E_{1}^{*}\right)
\end{array}\right]\right. \\
& \left.-[\bar{D}(\xi)-\bar{P}(x)]\left[\begin{array}{c}
\varepsilon_{32}^{*}+\mathrm{i} \varepsilon_{31}^{*} \\
-\frac{1}{2}\left(E_{2}^{*}+\mathrm{i} E_{1}^{*}\right)
\end{array}\right]+\mathrm{i} \sqrt{\frac{2}{\pi}} \boldsymbol{C}_{2}^{-1} \boldsymbol{K} \xi\right\}, \quad \xi \in \Omega_{1} ; \quad(14), \quad\left[\begin{array}{c}
\varepsilon_{32}^{*}+\mathrm{i} \varepsilon_{31}^{*} \\
-\frac{1}{2}\left(E_{2}^{*}+\mathrm{i} E_{1}^{*}\right)
\end{array}\right] \\
\boldsymbol{f}_{2}(\xi)= & \left(\boldsymbol{C}_{1}+\boldsymbol{C}_{2}\right)^{-1}\left(\boldsymbol{C}_{1}-\boldsymbol{C}_{2}\right) \\
& \times\left\{\left[\bar{D}(R / \xi)-\bar{P}(R / \xi)\left[\begin{array}{c}
\varepsilon_{32}^{*}-\mathrm{i} \varepsilon_{31}^{*} \\
-\frac{1}{2}\left(E_{2}^{*}-\mathrm{i} E_{1}^{*}\right)
\end{array}\right]-\mathrm{i} \sqrt{\frac{2}{\pi}} \boldsymbol{C}_{2}^{-1} \boldsymbol{K} R \xi^{-1}\right\}\right. \\
& \quad-[D(R / \xi)-P(R / \xi) \\
+ & {[D(\xi)-P(\xi)]\left[\begin{array}{c}
\varepsilon_{32}^{*}-\mathrm{i} \varepsilon_{31}^{*} \\
-\frac{1}{2}\left(E_{2}^{*}-\mathrm{i} E_{1}^{*}\right)
\end{array}\right]-[\bar{D}(\xi)-\bar{P}(\xi)]\left[\begin{array}{c}
\varepsilon_{32}^{*}+\mathrm{i} \varepsilon_{31}^{*} \\
-\frac{1}{2}\left(E_{2}^{*}+\mathrm{i} E_{1}^{*}\right)
\end{array}\right] } \\
+ & \mathrm{i} \sqrt{\frac{2}{\pi}} \boldsymbol{C}_{2}^{-1} \boldsymbol{K} \xi,
\end{aligned}
$$




$$
\begin{aligned}
\boldsymbol{f}_{3}(\xi)= & \left(\boldsymbol{C}_{1}+\boldsymbol{C}_{2}\right)^{-1}\left(\boldsymbol{C}_{1}-\boldsymbol{C}_{2}\right) \\
\times & \left\{[\bar{D}(R / \xi)-\bar{P}(R / \xi)]\left[\begin{array}{c}
\varepsilon_{32}^{*}+\mathrm{i} \varepsilon_{31}^{*} \\
-\frac{1}{2}\left(E_{2}^{*}+\mathrm{i} E_{1}^{*}\right)
\end{array}\right]\right. \\
& \left.-[D(R / \xi)-P(R / \xi)]\left[\begin{array}{c}
\varepsilon_{32}^{*}-\mathrm{i} \varepsilon_{31}^{*} \\
-\frac{1}{2}\left(E_{2}^{*}-\mathrm{i} E_{1}^{*}\right)
\end{array}\right]-\mathrm{i} \sqrt{\frac{2}{\pi}} \boldsymbol{C}_{2}^{-1} \boldsymbol{K} R \xi^{-1}\right\} \\
+ & \xi^{2}\left[\begin{array}{c}
\varepsilon_{32}^{*}+\mathrm{i} \varepsilon_{31}^{*} \\
-\frac{1}{2}\left(E_{2}^{*}+\mathrm{i} E_{1}^{*}\right)
\end{array}\right]-P(\xi)\left[\begin{array}{c}
\varepsilon_{32}^{*}-\mathrm{i} \varepsilon_{31}^{*} \\
-\frac{1}{2}\left(E_{2}^{*}-\mathrm{i} E_{1}^{*}\right)
\end{array}\right] \\
- & {[\bar{D}(\xi)-\bar{P}(\xi)]\left[\begin{array}{c}
\varepsilon_{32}^{*}+\mathrm{i} \varepsilon_{31}^{*} \\
-\frac{1}{2}\left(E_{2}^{*}+\mathrm{i} E_{1}^{*}\right)
\end{array}\right]+\mathrm{i} \sqrt{\frac{2}{\pi}} \boldsymbol{C}_{2}^{-1} \boldsymbol{K} \xi, \quad \xi \in \Omega_{3} . }
\end{aligned}
$$

It is not difficult to verify that the analytic vector functions obtained satisfy all the existing boundary and interface conditions as well as the required asymptotic behavior at infinity.

\section{Stress and electric displacement intensity factors}

The resultant stress and electric displacement intensity factors $K_{R}^{\sigma}$ and $K_{R}^{D}$ at the crack tip are defined by [Lee et al. 2000]

$$
K_{R}^{\sigma}=\lim _{x_{1} \rightarrow 0^{+}}\left[\sqrt{2 \pi x_{1}} \sigma_{32}\left(x_{1}, 0\right)\right], \quad K_{R}^{D}=\lim _{x_{1} \rightarrow 0^{+}}\left[\sqrt{2 \pi x_{1}} D_{2}\left(x_{1}, 0\right)\right],
$$

or equivalently

$$
\boldsymbol{K}_{R}=\left[\begin{array}{c}
K_{R}^{\sigma} \\
K_{R}^{D}
\end{array}\right]=\lim _{z \rightarrow 0}\left[\sqrt{2 \pi z} \boldsymbol{C}_{1} \boldsymbol{f}_{1}^{\prime}(z)\right]
$$

Using these definitions and (14), we ultimately obtain a concise and elegant expression of the resultant field intensity factors as

$$
\begin{aligned}
\boldsymbol{K}_{R}=2( & \left.\boldsymbol{C}_{1}^{-1}+\boldsymbol{C}_{2}^{-1}\right)^{-1} \boldsymbol{C}_{2}^{-1} \boldsymbol{K} \\
& +2 \sqrt{2 \pi}\left(C_{1}^{-1}+\boldsymbol{C}_{2}^{-2}\right)^{-1} \operatorname{Im}\left\{\left[D^{\prime}(0)-P^{\prime}(0)\right]\left[\begin{array}{c}
\varepsilon_{32}^{*}-\mathrm{i} \varepsilon_{31}^{*} \\
-\frac{1}{2}\left(E_{2}^{*}-\mathrm{i} E_{1}^{*}\right)
\end{array}\right]\right\} .
\end{aligned}
$$

We can see from the above expression that the intensity factors are independent of the radius of the circular inhomogeneity. Moreover, we can deduce that when the eigenstrains and eigenelectric fields satisfy the condition

$$
\frac{\varepsilon_{31}^{*}}{\varepsilon_{32}^{*}}=\frac{E_{1}^{*}}{E_{2}^{*}}=\frac{\operatorname{Im}\left\{D^{\prime}(0)-P^{\prime}(0)\right\}}{\operatorname{Re}\left\{D^{\prime}(0)-P^{\prime}(0)\right\}}
$$

Equation (19) gives us that

$$
\boldsymbol{K}_{R}=2\left(\boldsymbol{C}_{1}^{-1}+\boldsymbol{C}_{2}^{-1}\right)^{-1} \boldsymbol{C}_{2}^{-1} \boldsymbol{K},
$$




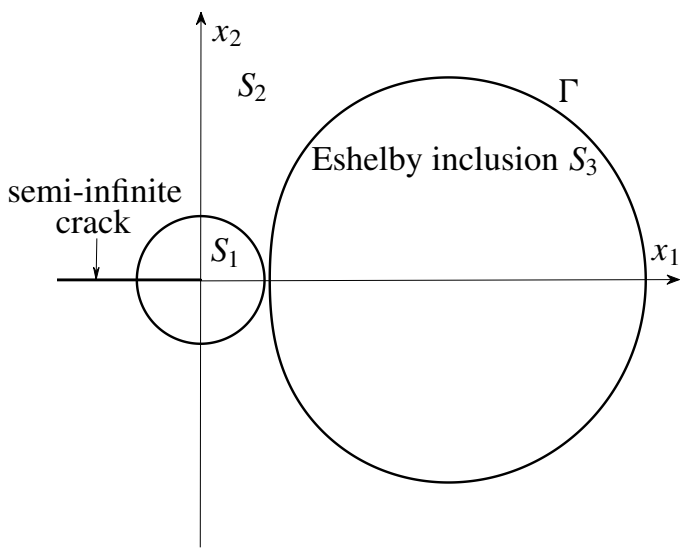

Figure 3. A noncircular interface $\Gamma$ when $\xi \in L$ is circular.

which implies that the Eshelby inclusion exerts no influence on the resultant field intensity factors at the crack tip, or equivalently exerts a neutral effect on the electroelastic field at the crack tip. Note that the condition in (20) is independent of the electroelastic constants of the fibrous piezoelectric composite.

\section{An illustrative example}

In this example, $\xi \in L$ is a circle described by

$$
\left|\xi-\xi_{0}\right|=d, \quad \xi \in L .
$$

Although $\xi \in L$ is a circle, $z \in \Gamma$ is noncircular. Such a noncircular interface $\Gamma$ is shown in Figure 3. The auxiliary function $D(\xi)$ and the polynomial $P(\xi)$ are found to be

$$
D(\xi)=\frac{d^{4}}{\left(\xi-\xi_{0}\right)^{2}}+\frac{2 \bar{\xi}_{0} d^{2}}{\xi-\xi_{0}}+\bar{\xi}^{2}, \quad P(\xi)=\bar{\xi}^{2} .
$$

Substituting these into (14)-(16), we obtain specific expressions for the three analytic vector functions as follows:

$$
\begin{aligned}
\boldsymbol{f}_{1}(\xi)=2\left(\boldsymbol{C}_{1}+\boldsymbol{C}_{2}\right)^{-1} \boldsymbol{C}_{2} \\
\times\left\{\left[\frac{d^{4}}{\left(\xi-\xi_{0}\right)^{2}}+\frac{2 \bar{\xi}_{0} d^{2}}{\xi-\xi_{0}}\right]\left[\begin{array}{c}
\varepsilon_{32}^{*}-\mathrm{i} \varepsilon_{31}^{*} \\
-\frac{1}{2}\left(E_{2}^{*}-\mathrm{i} E_{1}^{*}\right)
\end{array}\right]\right. \\
\quad-\left[\frac{d^{4}}{\left(\xi-\bar{\xi}_{0}\right)^{2}}+\frac{2 \xi_{0} d^{2}}{\xi-\bar{\xi}_{0}}\right]\left[\begin{array}{c}
\varepsilon_{32}^{*}+\mathrm{i} \varepsilon_{31}^{*} \\
-\frac{1}{2}\left(E_{2}^{*}+\mathrm{i} E_{1}^{*}\right)
\end{array}\right] \\
\left.+\mathrm{i} \sqrt{\frac{2}{\pi}} \boldsymbol{C}_{2}^{-1} \boldsymbol{K} \xi\right\}, \quad \xi \in \Omega_{1} ;
\end{aligned}
$$




$$
\begin{aligned}
& \boldsymbol{f}_{2}(\xi)=\left(\boldsymbol{C}_{1}+\boldsymbol{C}_{2}\right)^{-1}\left(\boldsymbol{C}_{1}-\boldsymbol{C}_{2}\right) \\
& \times\left\{\left[\frac{d^{4}}{\left(R \xi^{-1}-\bar{\xi}_{0}\right)^{2}}+\frac{2 \xi_{0} d^{2}}{R \xi^{-1}-\bar{\xi}_{0}}\right]\left[\begin{array}{c}
\varepsilon_{32}^{*}+\mathrm{i} \varepsilon_{31}^{*} \\
-\frac{1}{2}\left(E_{2}^{*}+\mathrm{i} E_{1}^{*}\right)
\end{array}\right]\right. \\
& -\left[\frac{d^{4}}{\left(R \xi^{-1}-\xi_{0}\right)^{2}}+\frac{2 \bar{\xi}_{0} d^{2}}{R \xi^{-1}-\xi_{0}}\right]\left[\begin{array}{c}
\varepsilon_{32}^{*}-\mathrm{i} \varepsilon_{31}^{*} \\
-\frac{1}{2}\left(E_{2}^{*}-\mathrm{i} E_{1}^{*}\right)
\end{array}\right] \\
& \left.-\mathrm{i} \sqrt{\frac{2}{\pi}} \boldsymbol{C}_{2}^{-1} K R \xi^{-1}\right\} \\
& +\left[\frac{d^{4}}{\left(\xi-\xi_{0}\right)^{2}}+\frac{2 \bar{\xi}_{0} d^{2}}{\xi-\xi_{0}}\right]\left[\begin{array}{c}
\varepsilon_{32}^{*}-\mathrm{i} \varepsilon_{31}^{*} \\
-\frac{1}{2}\left(E_{2}^{*}-\mathrm{i} E_{1}^{*}\right)
\end{array}\right] \\
& -\left[\frac{d^{4}}{\left(\xi-\bar{\xi}_{0}\right)^{2}}+\frac{2 \xi_{0} d^{2}}{\xi-\bar{\xi}_{0}}\right]\left[\begin{array}{c}
\varepsilon_{32}^{*}+\mathrm{i} \varepsilon_{31}^{*} \\
-\frac{1}{2}\left(E_{2}^{*}+\mathrm{i} E_{1}^{*}\right)
\end{array}\right]+\mathrm{i} \sqrt{\frac{2}{\pi}} \boldsymbol{C}_{2}^{-1} \boldsymbol{K} \xi, \quad \xi \in \Omega_{2} ; \\
& \boldsymbol{f}_{3}(\xi)=\left(\boldsymbol{C}_{1}+\boldsymbol{C}_{2}\right)^{-1}\left(\boldsymbol{C}_{1}-\boldsymbol{C}_{2}\right) \\
& \times\left\{\left[\frac{d^{4}}{\left(R \xi^{-1}-\bar{\xi}_{0}\right)^{2}}+\frac{2 \xi_{0} d^{2}}{R \xi^{-1}-\bar{\xi}_{0}}\right]\left[\begin{array}{c}
\varepsilon_{32}^{*}+\mathrm{i} \varepsilon_{31}^{*} \\
-\frac{1}{2}\left(E_{2}^{*}+\mathrm{i} E_{1}^{*}\right)
\end{array}\right]\right. \\
& -\left[\frac{d^{4}}{\left(R \xi^{-1}-\xi_{0}\right)^{2}}+\frac{2 \bar{\xi}_{0} d^{2}}{R \xi^{-1}-\xi_{0}}\right]\left[\begin{array}{c}
\varepsilon_{32}^{*}-\mathrm{i} \varepsilon_{31}^{*} \\
-\frac{1}{2}\left(E_{2}^{*}-\mathrm{i} E_{1}^{*}\right)
\end{array}\right] \\
& \left.-\mathrm{i} \sqrt{\frac{\pi}{2}} C_{2}^{-1} K R \xi^{-1}\right\} \\
& +\xi^{2}\left[\begin{array}{c}
\varepsilon_{32}^{*}+\mathrm{i} \varepsilon_{31}^{*} \\
-\frac{1}{2}\left(E_{2}^{*}+\mathrm{i} E_{1}^{*}\right)
\end{array}\right]-\bar{\xi}_{0}^{2}\left[\begin{array}{c}
\varepsilon_{32}^{*}-\mathrm{i} \varepsilon_{31}^{*} \\
-\frac{1}{2}\left(E_{2}^{*}-\mathrm{i} E_{1}^{*}\right)
\end{array}\right] \\
& -\left[\frac{d^{4}}{\left(\xi-\bar{\xi}_{0}\right)^{2}}+\frac{2 \xi_{0} d^{2}}{\xi-\bar{\xi}_{0}}\right]\left[\begin{array}{c}
\varepsilon_{32}^{*}+\mathrm{i} \varepsilon_{31}^{*} \\
-\frac{1}{2}\left(E_{2}^{*}+\mathrm{i} E_{1}^{*}\right)
\end{array}\right]+\mathrm{i} \sqrt{\frac{2}{\pi}} \boldsymbol{C}_{2}^{-1} \boldsymbol{K} \xi, \quad \xi \in \Omega_{3} .
\end{aligned}
$$

Substituting (23) into (19) yields

$$
\begin{aligned}
\boldsymbol{K}_{R}=2\left(\boldsymbol{C}_{1}^{-1}+\boldsymbol{C}_{2}^{-1}\right)^{-1} \boldsymbol{C}_{2}^{-1} \boldsymbol{K} & \\
+\frac{2 \sqrt{2 \pi} d^{2}\left(\left|\xi_{0}\right|^{2}-d^{2}\right)}{\left|\xi_{0}\right|^{6}}\left(\boldsymbol{C}_{1}^{-1}+\boldsymbol{C}_{2}^{-1}\right)^{-1} & \times\left[\begin{array}{c}
\left.2\left[\varepsilon_{31}^{*} u_{0}\left(u_{0}^{2}-3 v_{0}^{2}\right)+\varepsilon_{32}^{*} v_{0}\left(3 u_{0}^{2}-v_{0}^{2}\right)\right]\right], \\
-E_{1}^{*} u_{0}\left(u_{0}^{2}-3 v_{0}^{2}\right)-E_{2}^{*} v_{0}\left(3 u_{0}^{2}-v_{0}^{2}\right)
\end{array}\right],
\end{aligned}
$$

where

$$
\begin{aligned}
& u_{0}=\operatorname{Re}\left\{\xi_{0}\right\}, \\
& v_{0}=\operatorname{Im}\left\{\xi_{0}\right\} .
\end{aligned}
$$




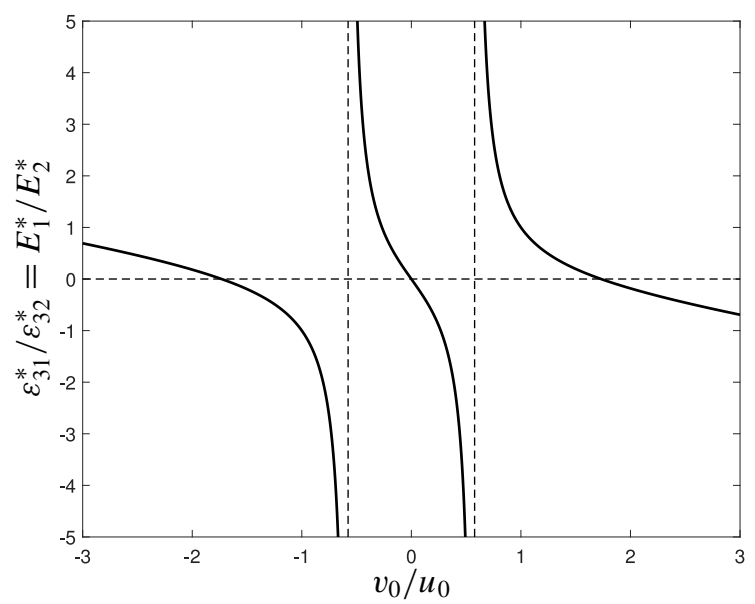

Figure 4. Variation of $\varepsilon_{31}^{*} / \varepsilon_{32}^{*}=E_{1}^{*} / E_{2}^{*}$ as a function of $v_{0} / u_{0}$ in (29).

When the eigenstrains and eigenelectric fields satisfy

$$
\frac{\varepsilon_{31}^{*}}{\varepsilon_{32}^{*}}=\frac{E_{1}^{*}}{E_{2}^{*}}=\frac{v_{0}\left(v_{0}^{2}-3 u_{0}^{2}\right)}{u_{0}\left(u_{0}^{2}-3 v_{0}^{2}\right)},
$$

the inclusion will have no influence on the resultant field intensity factors at the crack tip. Condition (29) can be deduced from (20) or indeed from (27). The variation of $\varepsilon_{31}^{*} / \varepsilon_{32}^{*}=E_{1}^{*} / E_{2}^{*}$ as a function of $v_{0} / u_{0}$ in (29) is illustrated in Figure 4. It is seen in Figure 4 that $\varepsilon_{31}^{*}=E_{1}^{*}=0$ when $v_{0} / u_{0}= \pm \sqrt{3}= \pm 1.7321$ and that $\varepsilon_{32}^{*}=E_{2}^{*}=0$ when $v_{0} / u_{0}= \pm 1 / \sqrt{3}= \pm 0.5774$.

Due to the fact that $\xi \in \Omega_{3}$ is circular, the average stresses and electric displacements within the Eshelby inclusion can be determined as

$$
\begin{aligned}
& {\left[\begin{array}{l}
\left\langle\sigma_{32}+\mathrm{i} \sigma_{31}\right\rangle \\
\left\langle D_{2}+\mathrm{i} D_{1}\right\rangle
\end{array}\right]} \\
& =-\mathrm{i} \sqrt{\frac{1}{2 \pi}} \boldsymbol{K} \xi_{0}^{-1}-\boldsymbol{C}_{2}\left[1+\frac{d^{2}\left(d^{2}-\left|\xi_{0}\right|^{2}+\xi_{0}^{2}\right)}{\xi_{0}\left(\xi_{0}-\bar{\xi}_{0}\right)^{3}}\right]\left[\begin{array}{c}
\varepsilon_{32}^{*}+\mathrm{i} \varepsilon_{31}^{*} \\
-\frac{1}{2}\left(E_{2}^{*}+\mathrm{i} E_{1}^{*}\right)
\end{array}\right] \\
& +\boldsymbol{C}_{2}\left(\boldsymbol{C}_{1}+\boldsymbol{C}_{2}\right)^{-1}\left(\boldsymbol{C}_{2}-\boldsymbol{C}_{1}\right) \\
& \times\left\{\frac{d^{2} R\left(d^{2}+R-\left|\xi_{0}\right|^{2}\right)}{\left(R-\left|\xi_{0}\right|^{2}\right)^{3}}\left[\begin{array}{c}
\varepsilon_{32}^{*}+\mathrm{i} \varepsilon_{31}^{*} \\
-\frac{1}{2}\left(E_{2}^{*}+\mathrm{i} E_{1}^{*}\right)
\end{array}\right]\right. \\
& -\frac{d^{2} R\left[\xi_{0}\left(d^{2}-\left|\xi_{0}\right|^{2}\right)+\bar{\xi}_{0} R\right]}{\xi_{0}\left(R-\xi_{0}^{2}\right)^{3}}\left[\begin{array}{c}
\varepsilon_{32}^{*}-\mathrm{i} \varepsilon_{31}^{*} \\
-\frac{1}{2}\left(E_{2}^{*}-\mathrm{i} E_{1}^{*}\right)
\end{array}\right] \\
& \left.+\mathrm{i} \sqrt{\frac{1}{2 \pi}} \boldsymbol{C}_{2}^{-1} \boldsymbol{K} R \xi_{0}^{-3}\right\}
\end{aligned}
$$

where $\langle\cdot\rangle$ denotes the average over $\xi \in \Omega_{3}$. 


\section{Conclusions}

We present a general method leading to an analytic solution of the interaction problem of an Eshelby inclusion of arbitrary shape undergoing uniform antiplane eigenstrains and in-plane eigenelectric fields near a circular piezoelectric inhomogeneity partially penetrated by a semi-infinite crack. The cracked piezoelectric plane in the physical $z$-plane is mapped onto the lower half of the image plane via the conformal mapping in (7). An auxiliary function $D(\xi)$ is constructed in (10). With the aid of $D(\xi)$, we apply analytic continuations across the straight boundary $\operatorname{Im}\{\xi\}=0$ and across the circular boundary $|\xi|=R^{1 / 2}$ to arrive at the three analytic vector functions $\boldsymbol{f}_{1}(\xi), \boldsymbol{f}_{2}(\xi)$, and $\boldsymbol{f}_{3}(\xi)$. A concise and elegant expression of the resultant stress and electric displacement intensity factors at the crack tip is obtained in (19). As an illustrative example, we present explicit expressions of the three analytic vector functions and the resultant intensity factors for the special case when $\xi \in L$ is a circle.

\section{Acknowledgements}

This work is supported by a grant from the National Natural Science Foundation of China (No. 11272121) and a Discovery Grant from the Natural Sciences and Engineering Research Council of Canada (RGPIN - 2017 - 03716115112).

\section{References}

[Chung and Ting 1996] M. Y. Chung and T. C. T. Ting, "Piezoelectric solid with an elliptic inclusion or hole", Int. J. Solids Struct. 33 (1996), 3343-3361.

[Deeg 1980] W. F. Deeg, The analysis of dislocation, crack, and inclusion problems in piezoelectric solids, Ph.D. thesis, Stanford University, Stanford, CA, 1980.

[England 1971] A. H. England, Complex variable methods in elasticity, Wiley, London, 1971.

[Kantorovich and Krylov 1958] L. V. Kantorovich and V. I. Krylov, Approximate methods in higher analysis, Interscience, New York, 1958.

[Kuo and Barnett 1991] C. M. Kuo and D. M. Barnett, "Stress singularities of interfacial cracks in bonded piezoelectric half-spaces", pp. 33-50 in Modern theory of anisotropic elasticity and applications (Research Triangle Park, NC, 1990), edited by J. J. Wu et al., 1991.

[Lee et al. 2000] K. Y. Lee, W. G. Lee, and Y. E. Pak, "Interaction between a semi-infinite crack and a screw dislocation in a piezoelectric material”, J. Appl. Mech. (ASME) 67 (2000), 165-170.

[Pak 1990] Y. E. Pak, "Crack extension force in a piezoelectric material”, J. Appl. Mech. (ASME) 57 (1990), 647-653.

[Ru 2000] C. Q. Ru, "Eshelby's problem for two-dimensional piezoelectric inclusions of arbitrary shape”, Proc. R. Soc. Lond. A 456:1997 (2000), 1051-1068.

[Ru 2001] C. Q. Ru, “A two-dimensional Eshelby problem for two bonded piezoelectric half-planes”, Proc. R. Soc. Lond. A 457:2008 (2001), 865-883.

[Savin 1961] G. N. Savin, Stress concentration around holes, Pergamon Press, London, 1961. 
[Suo et al. 1992] Z. Suo, C. M. Kuo, D. M. Barnett, and J. R. Willis, "Fracture mechanics for piezoelectric ceramics", J. Mech. Phys. Solids 40 (1992), 739-765.

[Wang and Fan 2015] X. Wang and H. Fan, "A piezoelectric screw dislocation in a bimaterial with surface piezoelectricity”, Acta Mech. 226 (2015), 3317-3331.

[Wang and Schiavone 2017] X. Wang and P. Schiavone, "Debonded arc-shaped interface conducting rigid line inclusions in piezoelectric composites”, Comptes Rendus Méc. 345:10 (2017), 724-731.

Received 3 Nov 2017. Revised 9 Jan 2018. Accepted 17 Feb 2018.

XU WANG: xuwang@ecust.edu.cn

School of Mechanical and Power Engineering, East China University of Science and Technology, Shanghai, China

PETER SCHIAVONE: p.schiavone@ualberta.ca

Department of Mechanical Engineering, University of Alberta, Edmonton, AB, Canada 


\section{Guidelines for Authors}

Authors may submit manuscripts in PDF format on-line at the submission page.

Originality. Submission of a manuscript acknowledges that the manuscript is original and and is not, in whole or in part, published or under consideration for publication elsewhere. It is understood also that the manuscript will not be submitted elsewhere while under consideration for publication in this journal.

Language. Articles in MEMOCS are usually in English, but articles written in other languages are welcome.

Required items. A brief abstract of about 150 words or less must be included. It should be selfcontained and not make any reference to the bibliography. If the article is not in English, two versions of the abstract must be included, one in the language of the article and one in English. Also required are keywords and a Mathematics Subject Classification or a Physics and Astronomy Classification Scheme code for the article, and, for each author, postal address, affiliation (if appropriate), and email address if available. A home-page URL is optional.

Format. Authors are encouraged to use $\mathrm{IAT}_{\mathrm{E} X}$ and the standard amsart class, but submissions in other varieties of $\mathrm{T}_{\mathrm{E}} \mathrm{X}$, and exceptionally in other formats, are acceptable. Initial uploads should normally be in PDF format; after the refereeing process we will ask you to submit all source material.

References. Bibliographical references should be complete, including article titles and page ranges. All references in the bibliography should be cited in the text. The use of $\mathrm{BIBT}_{\mathrm{E}} \mathrm{X}$ is preferred but not required. Tags will be converted to the house format, however, for submission you may use the format of your choice. Links will be provided to all literature with known web locations and authors are encouraged to provide their own links in addition to those supplied in the editorial process.

Figures. Figures must be of publication quality. After acceptance, you will need to submit the original source files in vector graphics format for all diagrams in your manuscript: vector EPS or vector PDF files are the most useful.

Most drawing and graphing packages - Mathematica, Adobe Illustrator, Corel Draw, MATLAB, etc. - allow the user to save files in one of these formats. Make sure that what you are saving is vector graphics and not a bitmap. If you need help, please write to graphics@msp.org with as many details as you can about how your graphics were generated.

Bundle your figure files into a single archive (using zip, tar, rar or other format of your choice) and upload on the link you been provided at acceptance time. Each figure should be captioned and numbered so that it can float. Small figures occupying no more than three lines of vertical space can be kept in the text ("the curve looks like this:"). It is acceptable to submit a manuscript with all figures at the end, if their placement is specified in the text by means of comments such as "Place Figure 1 here". The same considerations apply to tables.

White Space. Forced line breaks or page breaks should not be inserted in the document. There is no point in your trying to optimize line and page breaks in the original manuscript. The manuscript will be reformatted to use the journal's preferred fonts and layout.

Proofs. Page proofs will be made available to authors (or to the designated corresponding author) at a Web site in PDF format. Failure to acknowledge the receipt of proofs or to return corrections within the requested deadline may cause publication to be postponed. 
Mathematics and Mechanics of Complex Systems vol. 6 no. 2

Around two theorems and a lemma by Lucio Russo

Itai Benjamini and Gil Kalai

A strain gradient variational approach to damage: a comparison with damage gradient models and numerical results

Luca Placidi, Emilio Barchiesi and Anil Misra

On jets, almost symmetric tensors, and traction hyper-stresses

Reuven Segev and Jędrzej Śniatycki

An arbitrarily shaped Eshelby inclusion interacting with a circular piezoelectric inhomogeneity penetrated by a semi-infinite crack

$\mathrm{Xu}$ Wang and Peter Schiavone

MEMOCS is a journal of the International Research Center for the Mathematics and Mechanics of Complex Systems at the Università dell' Aquila, Italy.

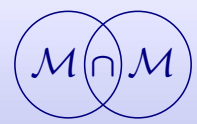

